\title{
Bioorthogonal retro-Cope elimination reaction of $N, N$-dialkylhydroxylamines and strained alkynes
}

Dahye Kang and Justin Kim*

Dana-Farber Cancer Institute, Department of Cancer Biology, Boston, Massachusetts 02215;

Harvard Medical School, Department of Biological Chemistry and Molecular Pharmacology, Boston, Massachusetts, 02115

\section{Supporting Material}

\begin{tabular}{lr} 
General Information & $\mathrm{S} 2$ \\
\hline
\end{tabular}

\begin{tabular}{lr} 
Materials & $\mathrm{S} 2$ \\
\hline
\end{tabular}

\begin{tabular}{ll} 
General Instrumentation & S2 \\
\hline
\end{tabular}

\begin{tabular}{ll} 
Synthetic Procedures and Characterization & S3 \\
\hline
\end{tabular}

\begin{tabular}{ll} 
Kinetics Studies & S20 \\
\hline
\end{tabular}

\begin{tabular}{lr} 
Protein Labeling Experiments & S23 \\
\hline
\end{tabular}

\begin{tabular}{ll} 
Intact Mass Spectrometry Analysis & S25 \\
\hline
\end{tabular}

\begin{tabular}{ll} 
Protein Labeling Experiments in the Presence of Cell Lysate & S26 \\
\hline
\end{tabular}

\begin{tabular}{lr}
\hline Stability Studies & S27 \\
\hline
\end{tabular}

\begin{tabular}{lr}
\hline Cross Reactivity Studies & S28 \\
\hline
\end{tabular}

\begin{tabular}{ll} 
Live Cell Dual Labeling Experiment & S31 \\
\hline
\end{tabular}

\begin{tabular}{ll} 
Intracellular Labeling Experiment & S35 \\
\hline
\end{tabular}

\begin{tabular}{lr} 
Cell Viability Assay & S37 \\
\hline
\end{tabular}

\begin{tabular}{lr}
\hline Computational Details & S38 \\
\hline References
\end{tabular}

\begin{tabular}{lr} 
References & S50 \\
\hline
\end{tabular}

\begin{tabular}{lr} 
Copies of NMR Spectra & S51 \\
\hline
\end{tabular} 
General Information. All reactions were conducted in flame-dried round-bottom flasks under a positive pressure of nitrogen unless otherwise stated. Gas-tight syringes with stainless steel needles or cannulae were used to transfer air- and moisture-sensitive liquids. Flash column chromatography was

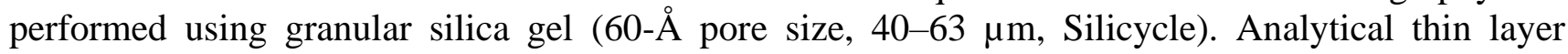
chromatography (TLC) was performed using glass plates pre-coated with $0.25 \mathrm{~mm}$ silica gel impregnated with a fluorescent indicator $(254 \mathrm{~nm}$, Silicycle). TLC plates were visualized by exposure to short wave ultraviolet light $(254 \mathrm{~nm})$ and/or an aqueous solution of potassium permanganate $\left(\mathrm{KMnO}_{4}, \mathrm{I}_{2}\right)$. Organic solutions were concentrated at $20{ }^{\circ} \mathrm{C}$ on rotary evaporators capable of achieving a minimum pressure of $\sim 2$ torr unless otherwise stated. Room temperature is defined as $22.5 \pm 2.5^{\circ} \mathrm{C}$. Reaction heating was performed using a UCON fluid heating bath.

Materials. All solvents were purchased from Fisher Scientific or Sigma-Aldrich. Unless otherwise stated chemical reagents were purchased from Fisher Scientific, Sigma-Aldrich, Alfa Aesar, Oakwood Chemical, Acros Organics, Combi-Blocks, or TCI America. CMA refers to a solution of 80:18:2 v/v/v chloroform:methanol:ammonium hydroxide (28-30\% ammonia solution). Chloroform used in CMA solutions and as co-eluents in silica gel column chromatography were stabilized with $0.75 \% \mathrm{v} / \mathrm{v}$ ethanol.

General Instrumentation. Proton nuclear magnetic resonance $\left({ }^{1} \mathrm{H}\right.$ NMR) spectra, recorded with a $500 \mathrm{MHz}$ Avance III Spectrometer with multi-nuclear Smart probe, are reported in parts per million on the $\delta$ scale, and are referenced from the residual protium in the NMR solvent $\left(\mathrm{CDCl}_{3}: \delta 7.24\right.$, $\mathrm{CD}_{3} \mathrm{OD}: \delta 3.31\left(\mathrm{CHD}_{2} \mathrm{OD}\right), \mathrm{CD}_{3} \mathrm{CN}: \delta$ 1.94). Data are reported as follows: chemical shift [multiplicity ( $\mathrm{s}=$ singlet, $\mathrm{d}=$ doublet, $\mathrm{t}=$ triplet, $\mathrm{dd}=$ doublet of doublets, $\mathrm{dt}=$ doublet of triplets, $\mathrm{dq}$ $=$ doublet of quartets, $\mathrm{ddd}=$ doublet of doublets of doublets, $\mathrm{tt}=$ triplet of triplets, $\mathrm{td}=$ triplet of doublets, $\mathrm{tq}=$ triplet of quatets, $\mathrm{m}=$ multiplet), coupling constant(s) in Hertz, integration, assignment]. Carbon-13 nuclear magnetic resonance $\left({ }^{13} \mathrm{C} \mathrm{NMR}\right)$ spectra are referenced from the carbon resonances of the solvent $\left(\mathrm{CDCl}_{3}: \delta 77.23, \mathrm{CD}_{3} \mathrm{OD}: \delta 49.15, \mathrm{CD}_{3} \mathrm{CN}: \delta\right.$ 1.37). Fluorine-19 nuclear magnetic resonance $\left({ }^{19} \mathrm{~F} \mathrm{NMR}\right)$ is calibrated from the fluorine resonances of benzotrifluoride $\left(\mathrm{CDCl}_{3}: \delta-62.76, \mathrm{CD}_{3} \mathrm{OD}: \delta-64.24\right)$. Data are reported as follows: chemical shift (assignment). Infrared data (IR) were obtained with a Cary 630 Fourier transform infrared spectrometer equipped with a diamond ATR objective and are reported as follows: frequency of absorption $\left(\mathrm{cm}^{-1}\right)$, intensity of absorption ( $\mathrm{s}=$ strong, $\mathrm{m}=$ medium, $\mathrm{w}=$ weak, $\mathrm{br}=$ broad). High resolution mass spectra (HRMS) were recorded on a Q Exactive ${ }^{\mathrm{TM}}$ Plus Hybrid Quadrupole-Orbitrap ${ }^{\mathrm{TM}}$ Mass Spectrometer using an electrospray ionization (ESI), atmospheric pressure ionization (API), or electron ionization (EI) source. Automated $\mathrm{C}_{18}$ reverse phase chromatography was performed using an Isolera One (Biotage) purification system. High performance liquid chromatography (HPLC) purification was performed using an Agilent 1260 Infinity system. In-gel fluorescence imaging was performed on a GE Healthcare Life Sciences Typhoon FLA 9500. Images were processed with Fiji ImageJ software. 


\section{Synthetic Procedures and Characterization}

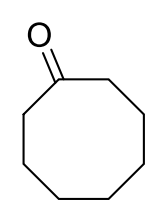

S1

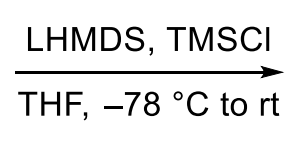

THF, $-78{ }^{\circ} \mathrm{C}$ to rt

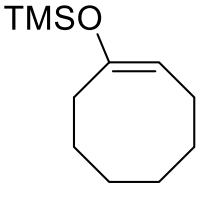

S2

\section{$\underline{(E)-(\text { cyclooct-1-en-1-yloxy)trimethylsilane (S2): }}$}

A round-bottom flask was charged sequentially with tetrahydrofuran $(200 \mathrm{~mL})$ and a solution of lithium bis(trimethylsilyl)amide (1 $\mathrm{M}$ in tetrahydrofuran, $52.3 \mathrm{~mL}, 52.3 \mathrm{mmol}, 1.10$ equiv) then cooled to $-78{ }^{\circ} \mathrm{C}$. A solution of cyclooctanone $\mathbf{S 1}(6.00 \mathrm{~g}, 47.5 \mathrm{mmol}, 1$ equiv) in tetrahydrofuran $(200 \mathrm{~mL})$ was added to the solution at $-78{ }^{\circ} \mathrm{C}$ via cannula over $20 \mathrm{~min}$. After $1.5 \mathrm{~h}$, chlorotrimethylsilane (TMSCl, $5.94 \mathrm{~g}, 54.7 \mathrm{mmol}, 1.15$ equiv) was added and the dry ice bath was removed. The solution was allowed to warm to room temperature. After $1 \mathrm{~h}$, the reaction was quenched with saturated aqueous ammonium chloride $(500 \mathrm{~mL})$ and diluted with hexanes $(500 \mathrm{~mL})$. The organic layer was washed with brine $(200 \mathrm{~mL})$, dried over anhydrous magnesium sulfate, filtered, and concentrated under reduced pressure. Crude $\mathbf{S 2}$ was used in the next step without further purification.

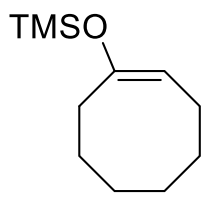

S2

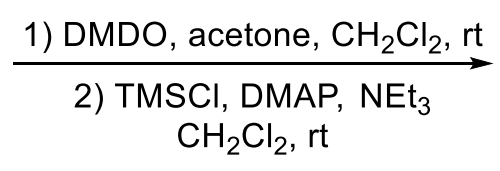

$82 \%$ over 3 steps

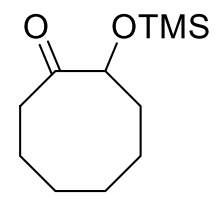

S3

\section{2-((trimethylsilyl)oxy)cyclooctan-1-one (S3):}

A round-bottom flask was charged with crude $\mathbf{S 2}$ from the previous step (47.5 mmol, 1 equiv) and dissolved in dichloromethane $(200 \mathrm{~mL})$. A solution of dimethyldioxirane (DMDO, $0.11 \mathrm{M}$ in acetone, $595 \mathrm{~mL}, 60.0 \mathrm{mmol}, 1.26$ equiv) was added to the solution at room temperature. After $15 \mathrm{~min}$, the reaction mixture was concentrated and azeotroped with methanol $(2 \times 200 \mathrm{~mL})$. The resulting oil was dissolved in dichloromethane $(500 \mathrm{~mL})$. 4-Dimethylaminopyridine (DMAP, $581 \mathrm{mg}, 4.75 \mathrm{mmol}$, 0.100 equiv), triethylamine $(9.94 \mathrm{~mL}, 71.3 \mathrm{mmol}, 1.50$ equiv), and chlorotrimethylsilane $(7.24 \mathrm{~mL}$, $57.1 \mathrm{mmol}, 1.2$ equiv) were sequentially added to the solution at room temperature. After $2.5 \mathrm{~h}$, the reaction mixture was washed with aqueous hydrogen chloride $(1 \mathrm{~N}, 500 \mathrm{~mL})$, the organic layer was separated, and the aqueous layer was extracted with dichloromethane $(75 \mathrm{~mL})$. The combined organic layers were dried over anhydrous sodium sulfate, filtered, and concentrated under reduced pressure. The crude mixture was purified by flash column chromatography on silica gel (eluent: 5\% ethyl acetate in hexanes) to provide ketone $\mathbf{S 3}$ (8.35 g, 82\% over 3 steps).

${ }^{1} \mathrm{H}$ NMR $\left(500 \mathrm{MHz}, \mathrm{CDCl}_{3}, 25{ }^{\circ} \mathrm{C}\right): \delta 4.15(\mathrm{dd}, J=6.9,3.4 \mathrm{~Hz}, 1 \mathrm{H}), 2.65-2.54(\mathrm{~m}, 1 \mathrm{H}), 2.32-2.20$ (m, 1H), 2.13-2.02 (m, 1H), 2.03-1.93 (m, 1H), 1.86-1.79 (m, 1H), 1.78-1.63 (m, 2H), 1.57-1.37 $(\mathrm{m}, 4 \mathrm{H}), 1.30-1.16(\mathrm{~m}, 1 \mathrm{H}), 0.08(\mathrm{~s}, 9 \mathrm{H})$. 
${ }^{13} \mathrm{C}$ NMR $\left(126 \mathrm{MHz}, \mathrm{CDCl}_{3}, 25{ }^{\circ} \mathrm{C}\right): \delta 217.6,77.7,39.1,34.7,27.2,26.1,25.3,21.4,0.2$.

FTIR (thin film) $\mathrm{cm}^{-1}: 2930$ (b), 1707 (w), 1252 (m), 1111 (m), 1051 (m), 835 (s).

HRMS (ESI) $(m / z)$ : calc'd for $\mathrm{C}_{11} \mathrm{H}_{23} \mathrm{O}_{2} \mathrm{Si}[\mathrm{M}+\mathrm{H}]^{+}:$215.1467, found: 215.1463 .

TLC (5\% ethyl acetate in hexanes), $\mathrm{R} f: 0.70\left(\mathrm{I}_{2}\right)$.

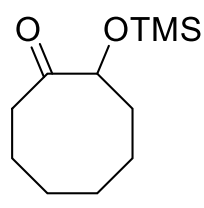

S3

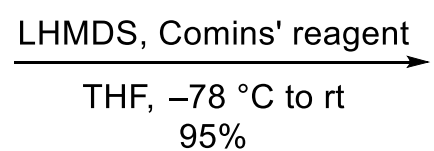

$95 \%$

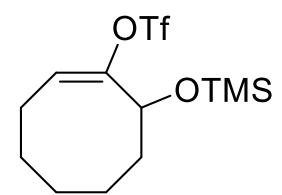

S4

\section{(E)-8-((trimethylsilyl)oxy)cyclooct-1-en-1-yl trifluoromethanesulfonate (S4):}

A round-bottom flask was charged sequentially with cyclooctanone $\mathbf{S 3}$ (2.06 g, $9.61 \mathrm{mmol}, 1$ equiv) and tetrahydrofuran $(100 \mathrm{~mL})$ then cooled to $-78^{\circ} \mathrm{C}$. A solution of lithium bis(trimethylsilyl)amide $(1$ $\mathrm{M}$ in tetrahydrofuran, $11.5 \mathrm{~mL}, 11.5 \mathrm{mmol}, 1.20$ equiv) at $-78{ }^{\circ} \mathrm{C}$ was added to the mixture via cannula. After $1 \mathrm{~h}, \mathrm{~N}$-(5-chloro-2-pyridyl)bis(trifluoromethanesulfonimide) (4.15 g, $10.6 \mathrm{mmol}, 1.10$ equiv) was added, and the dry ice bath was removed. After $2 \mathrm{~h}$, the reaction mixture was diluted with hexanes $(200 \mathrm{~mL})$ and washed sequentially with aqueous sodium hydroxide $(1 \mathrm{M}, 2 \times 150 \mathrm{~mL})$ and brine $(100 \mathrm{~mL})$. The resulting organic layer was dried over anhydrous sodium sulfate, filtered, and concentrated under reduced pressure. The crude mixture was purified by flash column chromatography on silica gel (eluent: $7.5 \%$ dichloromethane in hexanes) to provide vinyl triflate $\mathbf{S 4}$ $(3.1 \mathrm{~g}, 95 \%)$ as a colorless oil.

${ }^{1} \mathrm{H}$ NMR $\left(500 \mathrm{MHz}, \mathrm{CDCl}_{3}, 25^{\circ} \mathrm{C}\right): \delta 5.68(\mathrm{t}, J=9.0 \mathrm{~Hz}, 1 \mathrm{H}), 4.66(\mathrm{dd}, J=10.3,5.4 \mathrm{~Hz}, 1 \mathrm{H}), 2.37-$ $2.21(\mathrm{~m}, 1 \mathrm{H}), 2.11-1.97(\mathrm{~m}, 1 \mathrm{H}), 1.83-1.67(\mathrm{~m}, 4 \mathrm{H}), 1.65-1.53(\mathrm{~m}, 1 \mathrm{H}), 1.52-1.29(\mathrm{~m}, 3 \mathrm{H}), 0.14(\mathrm{~s}$, $9 \mathrm{H})$.

${ }^{13} \mathrm{C} \mathrm{NMR}\left(126 \mathrm{MHz}, \mathrm{CDCl}_{3}, 25^{\circ} \mathrm{C}\right): \delta 150.6,118.8(\mathrm{q}, J=319.6 \mathrm{~Hz}), 120.0,67.3,37.0,29.9,26.2$, 24.8, 23.6, -0.1.

${ }^{19} \mathrm{~F}$ NMR (471 MHz, $\left.\mathrm{CDCl}_{3}, 25{ }^{\circ} \mathrm{C}\right): \delta-75.2$.

FTIR (thin film) $\mathrm{cm}^{-1}: 2933$ (w), 1416 (m), 1200 (s), 1144 (m), 932 (m), 839 (s).

HRMS (ESI) $(m / z)$ : calc'd for $\mathrm{C}_{12} \mathrm{H}_{21} \mathrm{~F}_{3} \mathrm{NaO}_{4} \mathrm{SSi}[\mathrm{M}+\mathrm{Na}]^{+}:$369.0774, found: 369.0776.

TLC (100\% hexanes), Rf: $0.42\left(\mathrm{I}_{2}\right)$. 


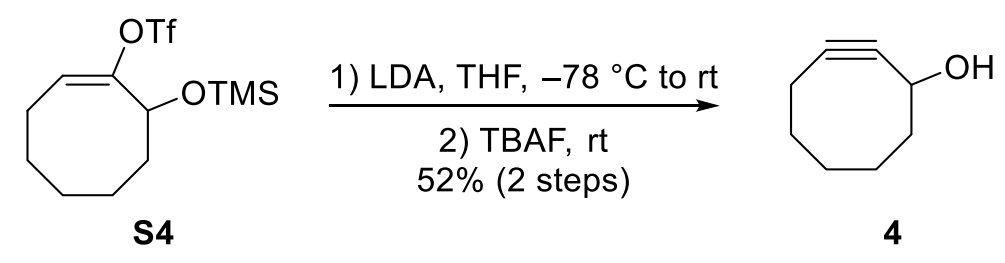

\section{cyclooct-2-yn-1-ol (4):}

A round-bottom flask was charged sequentially with vinyl triflate $\mathbf{S 4}$ (3.03 g, $8.75 \mathrm{mmol}, 1$ equiv) and tetrahydrofuran $(88 \mathrm{~mL})$ then cooled to $-78^{\circ} \mathrm{C}$. A solution of lithium diisopropylamide $(2.0 \mathrm{M}$ in tetrahydrofuran/heptane/ethylbenzene, $8.75 \mathrm{~mL}, 17.5 \mathrm{mmol}, 2.00$ equiv) was then added to the solution via syringe. The dry ice bath was removed, and the solution was allowed to warm to room temperature. After $2.5 \mathrm{~h}$, tetrabutylammonium fluoride $(1 \mathrm{M}$ in tetrahydrofuran, $17.5 \mathrm{~mL}, 17.5 \mathrm{mmol}$, 2.00 equiv) was added to the reaction mixture via syringe. After $1 \mathrm{~h}$, the reaction mixture was diluted with hexanes $(100 \mathrm{~mL})$ and washed with saturated aqueous ammonium chloride $(100 \mathrm{~mL})$ and brine $(100 \mathrm{~mL})$. The organic layer was dried over anhydrous sodium sulfate, filtered, and concentrated under reduced pressure. The crude mixture was purified by flash column chromatography on silica gel (eluent: $100 \%$ dichloromethane) to provide cyclooctynol 4 (566 mg, 52\%) as a clear, colorless oil. The physical properties and spectral data were identical to those reported in the literature. ${ }^{1}$

TLC (100\% dichloromethane), $\mathrm{R} f: 0.31\left(\mathrm{KMnO}_{4}\right)$.

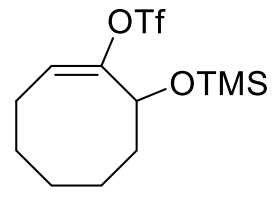

S4

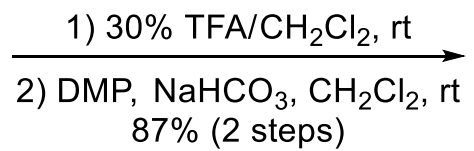
$87 \%$ (2 steps)

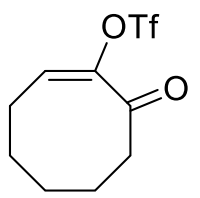

S5

\section{$\underline{(E)-8-0 x 0 c y c l o o c t-1-e n-1-y l ~ t r i f l u o r o m e t h a n e s u l f o n a t e ~(S 5): ~}$}

A round-bottom flask was charged sequentially with vinyl triflate $\mathbf{S 4}$ (94.3 $\mathrm{mg}, 272 \mu \mathrm{mol}, 1$ equiv) and dichloromethane $(1.4 \mathrm{~mL})$. Trifluoroacetic acid $(600 \mu \mathrm{L})$ was added to the solution at room temperature. After $30 \mathrm{~min}$, the reaction mixture was concentrated under reduced pressure. The crude mixture was dissolved in dichloromethane $(2.7 \mathrm{~mL})$. Sodium bicarbonate $(68.6 \mathrm{mg}, 817 \mu \mathrm{mol}, 3.00$ equiv) and Dess-Martin periodinane (DMP, $231 \mathrm{mg}, 544 \mu \mathrm{mol}, 2.00$ equiv) were sequentially added at room temperature. After $30 \mathrm{~min}$, the reaction mixture was diluted with hexanes $(2 \mathrm{~mL})$ and purified by flash column chromatography on silica gel (eluent: $15 \%$ ethyl acetate in hexanes) to provide cyclooctenone $\mathbf{S 5}$ (64.1 mg, 87\%) as a clear thin film.

${ }^{1} \mathrm{H}$ NMR $\left(500 \mathrm{MHz}, \mathrm{CDCl}_{3}, 25^{\circ} \mathrm{C}\right): \delta 6.58(\mathrm{t}, J=9.2 \mathrm{~Hz}, 1 \mathrm{H}), 2.85(\mathrm{t}, J=7.3 \mathrm{~Hz}, 2 \mathrm{H}), 2.70(\mathrm{dt}, J=$ 9.3, 7.0 Hz, 2H), 1.84-1.75 (m, 2H), 1.74-1.68 (m, 2H), 1.61-1.54 (m, 2H).

${ }^{13} \mathrm{C}$ NMR $\left(126 \mathrm{MHz}, \mathrm{CDCl}_{3}, 25{ }^{\circ} \mathrm{C}\right): \delta 192.8,149.7,133.6,118.8(\mathrm{q}, J=320.1 \mathrm{~Hz}), 40.7,25.3,23.5$, 23.1, 22.0.

${ }^{19} \mathrm{~F}$ NMR $\left(471 \mathrm{MHz}, \mathrm{CDCl}_{3}, 25{ }^{\circ} \mathrm{C}\right): \delta-74.2$. 
FTIR (thin film) $\mathrm{cm}^{-1}: 2937$ (w), 1685 (m), 1416 (s), 1200 (s), 1141 (s), 1062 (s), 969 (s).

HRMS (ESI) $(m / z)$ : calc'd for $\mathrm{C}_{9} \mathrm{H}_{12} \mathrm{~F}_{3} \mathrm{O}_{4} \mathrm{~S}[\mathrm{M}+\mathrm{H}]^{+}: 273.0403$, found: 273.0402 .

TLC (15\% ethyl acetate in hexanes), Rf: $0.30\left(\mathrm{KMnO}_{4}\right)$.

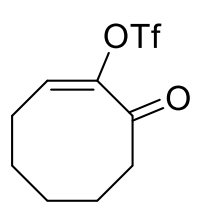

S5

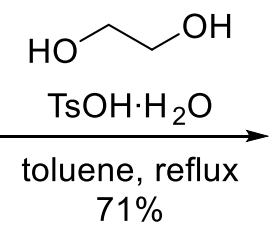

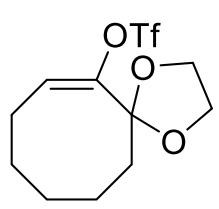

56

\section{(E)-1,4-dioxaspiro[4.7]dodec-6-en-6-yl trifluoromethanesulfonate (S6):}

A round-bottom flask was sequentially charged with cyclooctenone $\mathbf{S 5}$ (150 mg, $551 \mu \mathrm{mol}, 1$ equiv), ethylene glycol $(302 \mu \mathrm{L}, 5.51 \mathrm{mmol}, 10.0$ equiv), and benzene $(10 \mathrm{~mL})$ at room temperature. $p$ Toluenesulfonic acid monohydrate $(10.5 \mathrm{mg}, 55.1 \mu \mathrm{mol}, 0.100$ equiv) was then added to the solution. The flask was fitted with a Dean-Stark trap and reflux condenser, and the reaction mixture was heated to reflux. After $23 \mathrm{~h}$, the reaction mixture was cooled to room temperature and diluted with hexanes. The crude mixture was purified by flash column chromatography on silica gel (eluent: 5\% ethyl acetate in hexanes) to provide ketal $\mathbf{S 6}(125 \mathrm{mg}, 71 \%)$ as a clear, colorless oil.

${ }^{1} \mathrm{H}$ NMR $\left(500 \mathrm{MHz}, \mathrm{CDCl}_{3}, 25^{\circ} \mathrm{C}\right): \delta 5.75(\mathrm{t}, J=9.5 \mathrm{~Hz}, 1 \mathrm{H}), 4.14-3.91(\mathrm{~m}, 4 \mathrm{H}), 2.48-2.36(\mathrm{~m}$, 2H), 2.04-1.98 (m, 2H), 1.65-1.52 (m, 6H).

${ }^{13} \mathrm{C}$ NMR $\left(126 \mathrm{MHz}, \mathrm{CDCl}_{3}, 25{ }^{\circ} \mathrm{C}\right): \delta 150.1,123.0,118.7(\mathrm{q}, J=319.5 \mathrm{~Hz}), 107.3,65.7,37.7,27.2$, 23.1, 22.7, 21.9.

${ }^{19} \mathrm{~F}$ NMR $\left(471 \mathrm{MHz}, \mathrm{CDCl}_{3}, 25^{\circ} \mathrm{C}\right): \delta-75.4$.

FTIR (thin film) $\mathrm{cm}^{-1}$ : 2930 (w), 1409 (s), 1245 (w), 1200 (s), 1141 (s), 977 (s).

HRMS (ESI) $(m / z)$ : calc'd for $\mathrm{C}_{11} \mathrm{H}_{16} \mathrm{~F}_{3} \mathrm{O}_{5} \mathrm{~S}[\mathrm{M}+\mathrm{H}]^{+}: 317.0665$, found: 317.0664 .

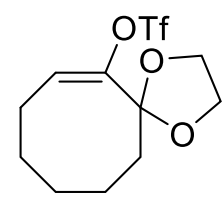

S6

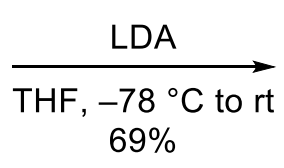

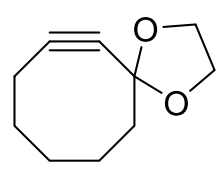

5

\section{1,4-dioxaspiro[4.7]dodec-6-yne (5):}

A round-bottom flask was sequentially charged with ketal S6 (70.1 mg, $222 \mu \mathrm{mol}, 1$ equiv) and tetrahydrofuran $(4 \mathrm{~mL})$ then cooled to $-78{ }^{\circ} \mathrm{C}$. A solution of lithium diisopropylamide (LDA, $2 \mathrm{M}$ in 
tetrahydrofuran/heptane/ethylbenzene, $222 \mu \mathrm{L}, 443 \mu \mathrm{mol}, 2.00$ equiv) was added to the solution via syringe. The dry ice bath was immediately removed, and the solution was allowed to warm to room temperature. After $2.5 \mathrm{~h}$, the solution was cooled to $-78{ }^{\circ} \mathrm{C}$ and additional lithium diisopropylamide solution ( $2 \mathrm{M}$ in tetrahydrofuran/heptane/ethylbenzene, $111 \mu \mathrm{L}, 222 \mu \mathrm{mol}, 1.00$ equiv) was added via syringe. The dry ice bath was removed, and the solution was allowed to warm to room temperature. After $1.5 \mathrm{~h}$, the reaction was quenched with methanol $(1.0 \mathrm{~mL})$ and concentrated under reduced pressure. The crude mixture was purified by flash column chromatography on silica gel (eluent: $5 \%$ ethyl acetate in hexanes) to provide cyclooctyne $\mathbf{5}(25.5 \mathrm{mg}, 69 \%)$ as a clear thin film.

${ }^{1} \mathrm{H}$ NMR $\left(500 \mathrm{MHz}, \mathrm{CDCl}_{3}, 25^{\circ} \mathrm{C}\right): \delta 3.98-3.84(\mathrm{~m}, 4 \mathrm{H}), 2.22(\mathrm{t}, J=6.4 \mathrm{~Hz}, 2 \mathrm{H}), 2.18-2.11(\mathrm{~m}$, $2 \mathrm{H}), 1.95-1.87(\mathrm{~m}, 2 \mathrm{H}), 1.77-1.71(\mathrm{~m}, 2 \mathrm{H}), 1.68-1.61(\mathrm{~m}, 2 \mathrm{H})$.

${ }^{13} \mathrm{C} \mathrm{NMR}\left(126 \mathrm{MHz}, \mathrm{CDCl}_{3}, 25{ }^{\circ} \mathrm{C}\right): \delta 107.4,105.3,89.8,64.7,47.4,34.2,29.8,27.0,20.6$.

FTIR (thin film) $\mathrm{cm}^{-1}$ : 2926 (m), 2214 (w), 1446 (w) 1275 (w), 1170 (m), 1129 (s), 1029 (s).

HRMS (ESI) $(m / z)$ : calc'd for $\mathrm{C}_{10} \mathrm{H}_{15} \mathrm{O}_{2}[\mathrm{M}+\mathrm{H}]^{+}:$167.1067, found: 167.1067 .

TLC (5\% ethyl acetate in hexanes) $\mathrm{R} f: 0.38\left(\mathrm{KMnO}_{4}\right)$.

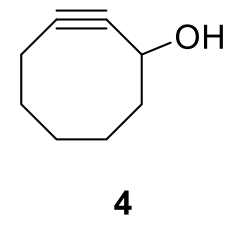

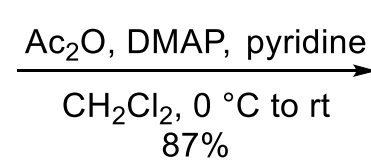

$87 \%$

\section{cyclooct-2-yn-1-yl acetate (6):}

A round-bottom flask was sequentially charged with cyclooctynol 4 (80.5 mg, $648 \mu \mathrm{mol}, 1$ equiv), 4dimethylaminopyridine $(6.3 \mathrm{mg}, 51.9 \mu \mathrm{mol}, 0.08$ equiv), and dichloromethane $(3.0 \mathrm{~mL})$ at room temperature. The solution was then cooled to $0{ }^{\circ} \mathrm{C}$ with an ice-water bath, and pyridine $(261 \mu \mathrm{L}, 3.24$ mmol, 5.00 equiv) was added dropwise to the solution. Acetic anhydride $(73.5 \mu \mathrm{L}, 778 \mu \mathrm{mol}, 1.20$ equiv) was then added dropwise to the solution. The ice bath was removed, and the solution was allowed to warm to room temperature. After $16 \mathrm{~h}$, the reaction was quenched with saturated aqueous ammonium chloride $(1 \mathrm{~mL})$ and diluted with dichloromethane $(50 \mathrm{~mL})$. The organic layer was washed with water $(50 \mathrm{~mL})$, dried over anhydrous magnesium sulfate, and concentrated under reduced pressure. The crude mixture was purified by flash column chromatography on silica gel (eluent: $50 \%$ dichloromethane in hexanes) to provide cyclooctyne $6(95.5 \mathrm{mg}, 87 \%)$ as a clear, colorless oil.

${ }^{1} \mathrm{H}$ NMR $\left(500 \mathrm{MHz}, \mathrm{CDCl}_{3}, 25^{\circ} \mathrm{C}\right): \delta$ 5.34-5.26 (m, 1H), 2.31-2.21 (m, 1H), 2.21-2.08 (m, 2H), $2.02(\mathrm{~s}, 3 \mathrm{H}), 2.02-1.93(\mathrm{~m}, 1 \mathrm{H}), 1.94-1.83(\mathrm{~m}, 2 \mathrm{H}), 1.83-1.72(\mathrm{~m}, 1 \mathrm{H}), 1.72-1.57(\mathrm{~m}, 2 \mathrm{H}), 1.57-$ $1.46(\mathrm{~m}, 1 \mathrm{H})$.

${ }^{13} \mathrm{C} \mathrm{NMR}\left(126 \mathrm{MHz}, \mathrm{CDCl}_{3}, 25{ }^{\circ} \mathrm{C}\right): \delta 170.4,102.0,90.8,66.7,41.7,34.4,29.8,26.4,21.3,20.9$.

FTIR (thin film) $\mathrm{cm}^{-1}: 2930$ (m), 1737 (s), 1450 (w), 1230 (s), 1025 (m), 969 (m). 
HRMS (ESI) $(m / z)$ : calc'd for $\mathrm{C}_{10} \mathrm{H}_{15} \mathrm{O}_{2}[\mathrm{M}+\mathrm{H}]^{+}:$167.1067, found: 167.1068 .

TLC $\left(100 \% \mathrm{CH}_{2} \mathrm{Cl}_{2}\right), \mathrm{R} f: 0.57\left(\mathrm{I}_{2}\right)$.

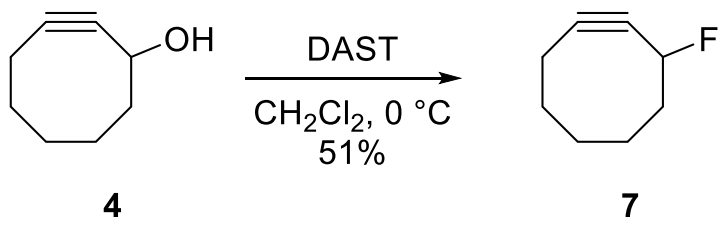

\section{3-fluorocyclooct-1-yne (7):}

A round-bottom flask was sequentially charged with cyclooctynol 4 (40.8 mg, $329 \mu \mathrm{mol}, 1$ equiv) and dichloromethane $(3.0 \mathrm{~mL})$ then cooled to $0{ }^{\circ} \mathrm{C}$. Diethylaminosulfur trifluoride (DAST, $45.6 \mu \mathrm{L}$, $345 \mu \mathrm{mol}, 1.05$ equiv) was then added to the solution via syringe. After $1 \mathrm{~h}$, the reaction mixture was concentrated under reduced pressure. The crude mixture was purified by flash column chromatography on silica gel (eluent: $100 \%$ pentane) to provide fluorocyclooctyne $7(21.0 \mathrm{mg}, 51 \%)$ as a clear, colorless oil.

${ }^{1} \mathrm{H}$ NMR $\left(500 \mathrm{MHz}, \mathrm{CDCl}_{3}, 25{ }^{\circ} \mathrm{C}\right): \delta 5.12(\mathrm{dt}, J=50.5,5.1 \mathrm{~Hz}, 1 \mathrm{H}), 2.34-2.01(\mathrm{~m}, 4 \mathrm{H}), 1.94-1.86$ $(\mathrm{m}, 2 \mathrm{H}), 1.82-1.68(\mathrm{~m}, 2 \mathrm{H}), 1.64-1.44(\mathrm{~m}, 2 \mathrm{H})$.

${ }^{13} \mathrm{C}$ NMR $\left(126 \mathrm{MHz}, \mathrm{CDCl}_{3}, 25{ }^{\circ} \mathrm{C}\right): \delta 104.9(\mathrm{~d}, J=10.5 \mathrm{~Hz}), 90.5(\mathrm{~d}, J=30.0 \mathrm{~Hz}), 84.7(\mathrm{~d}, J=$ $171.2 \mathrm{~Hz}), 43.0(\mathrm{~d}, J=22.9 \mathrm{~Hz}), 34.3$ (d, $J=1.9 \mathrm{~Hz}), 29.6,25.5(\mathrm{~d}, J=2.9 \mathrm{~Hz}), 20.9$ (d, $J=2.9 \mathrm{~Hz})$.

${ }^{19} \mathrm{~F}$ NMR (471 MHz, $\left.\mathrm{CDCl}_{3}, 25^{\circ} \mathrm{C}\right): \delta-172.2$.

FTIR (thin film) $\mathrm{cm}^{-1}$ : 2930 (s), 2855 (m), 2214 (w), 1450 (m), 1353 (m), 1029 (m), 988 (s).

HRMS (ESI) $(m / z)$ : calc'd for $\mathrm{C}_{8} \mathrm{H}_{12} \mathrm{~F}[\mathrm{M}+\mathrm{H}]^{+}:$127.0918, found: 127.0916 .

TLC (100\% pentane), $\mathrm{R} f: 0.26\left(\mathrm{KMnO}_{4}\right)$.

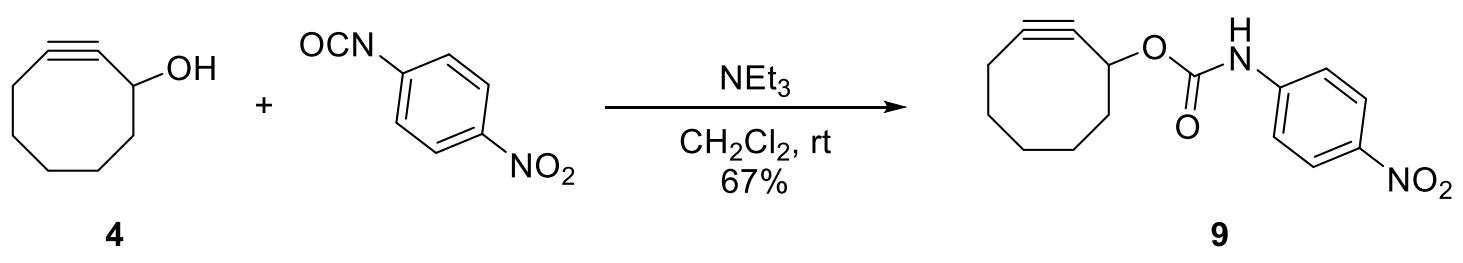

\section{cyclooct-2-yn-1-yl (4-nitrophenyl)carbamate (9):}

A round-bottom flask was sequentially charged with cyclooctynol 4 (10.8 mg, $87.0 \mu \mathrm{mol}, 1$ equiv) and dichloromethane $(1 \mathrm{~mL})$. 1-Isocyanato-4-nitrobenzene $(14.3 \mathrm{mg}, 87.0 \mu \mathrm{mol}, 1.00 \mathrm{equiv})$ and triethylamine $(1.2 \mu \mathrm{L}, 8.70 \mu \mathrm{mol}, 0.100$ equiv $)$ were added to the solution at room temperature. After 
$1 \mathrm{~h}$ and $40 \mathrm{~min}$, the reaction mixture was diluted with hexanes. The crude mixture was purified by flash column chromatography on silica gel (eluent: $30 \%$ diethyl ether in hexanes) to provide carbamate 9 (16.7 $\mathrm{mg}, 67 \%)$ as a white solid.

${ }^{1} \mathrm{H}$ NMR $\left(500 \mathrm{MHz}, \mathrm{CD}_{3} \mathrm{CN}, 25{ }^{\circ} \mathrm{C}\right): \delta 8.26(\mathrm{~s}, 1 \mathrm{H}), 8.16(\mathrm{~d}, J=9.3 \mathrm{~Hz}, 2 \mathrm{H}), 7.62(\mathrm{~d}, J=9.3 \mathrm{~Hz}$, $2 \mathrm{H}), 5.32(\mathrm{tq}, J=5.1,2.2 \mathrm{~Hz}, 1 \mathrm{H}), 2.35-2.24(\mathrm{~m}, 1 \mathrm{H}), 2.24-2.15(\mathrm{~m}, 2 \mathrm{H}), 2.08-1.99(\mathrm{~m}, 1 \mathrm{H}), 1.94-$ $1.86(\mathrm{~m}, 2 \mathrm{H}), 1.86-1.75(\mathrm{~m}, 1 \mathrm{H}), 1.73-1.63(\mathrm{~m}, 2 \mathrm{H}), 1.64-1.52(\mathrm{~m}, 1 \mathrm{H})$.

${ }^{13} \mathrm{C}$ NMR $\left(126 \mathrm{MHz}, \mathrm{CD}_{3} \mathrm{CN}, 25{ }^{\circ} \mathrm{C}\right): \delta 153.5,146.1,143.8,126.0,118.8,103.2,91.6,68.7,42.5$, $35.0,30.4,26.9,21.1$.

FTIR (thin film) $\mathrm{cm}^{-1}: 3321$ (m), 2930 (m), 1722 (s), 1566 (s), 1510 (s), 1327 (s), 1226 (s), 1055 (s).

HRMS (ESI) $(m / z)$ : calc'd for $\mathrm{C}_{15} \mathrm{H}_{17} \mathrm{~N}_{2} \mathrm{O}_{4}[\mathrm{M}+\mathrm{H}]^{+}: 289.1183$, found: 289.1188 .

TLC (50\% dichloromethane in hexanes), $\mathrm{R} f: 0.23\left(\mathrm{KMnO}_{4}\right)$.

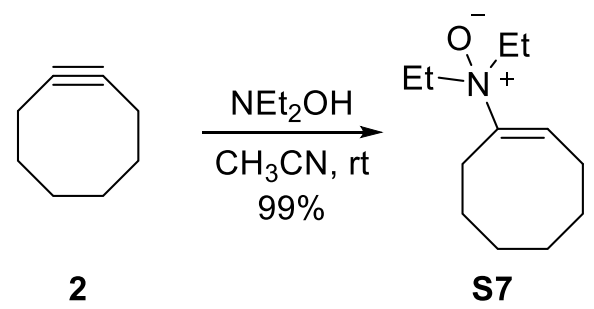

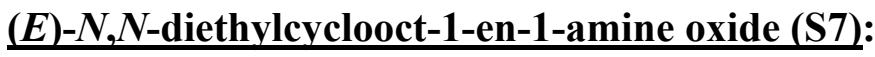

$N, N$-Diethylhydroxylamine $(28.4 \mu \mathrm{L}, 276 \mu \mathrm{mol}, 1.50$ equiv) was added via syringe to a solution of cyclooctyne $\mathbf{2}^{2}(19.9 \mathrm{mg}, 184 \mu \mathrm{mol}, 1$ equiv) in acetonitrile $(1.8 \mathrm{~mL})$ at room temperature. After 10 min, the reaction mixture was concentrated under reduced pressure. The crude mixture was purified by flash column chromatography on silica gel (eluent: $15 \rightarrow 30 \% \mathrm{CMA}$ in chloroform) to provide enamine $N$-oxide $\mathbf{S 7}$ (36.0 $\mathrm{mg}, 99 \%)$ as a clear thin film.

${ }^{1} \mathrm{H}$ NMR (500 MHz, $\left.\mathrm{CDCl}_{3}, 25^{\circ} \mathrm{C}\right): \delta 6.65(\mathrm{t}, J=8.8 \mathrm{~Hz}, 1 \mathrm{H}), 3.40-3.09(\mathrm{~m}, 4 \mathrm{H}), 2.41-2.27(\mathrm{~m}$, 2H), 2.19-2.09 (m, 2H), 1.67-1.39 (m, 8H), $1.16(\mathrm{t}, J=7.1 \mathrm{~Hz}, 6 \mathrm{H})$.

${ }^{13} \mathrm{C} \mathrm{NMR}\left(126 \mathrm{MHz}, \mathrm{CDCl}_{3}, 25{ }^{\circ} \mathrm{C}\right): \delta 146.8,125.6,61.8,29.7,28.3,26.1,26.0,25.6,25.3,8.8$.

FTIR (thin film) $\mathrm{cm}^{-1}: 3340$ (br), 2926 (s), 2855 (m), 1655 (w), 1466 (m), 956 (s).

HRMS (ESI) $(m / z)$ : calc'd for $\mathrm{C}_{12} \mathrm{H}_{24} \mathrm{NO}[\mathrm{M}+\mathrm{H}]^{+}:$198.1852, found: 198.1853.

TLC (50\% CMA in chloroform), $\mathrm{R} f: 0.38\left(\mathrm{KMnO}_{4}\right)$. 


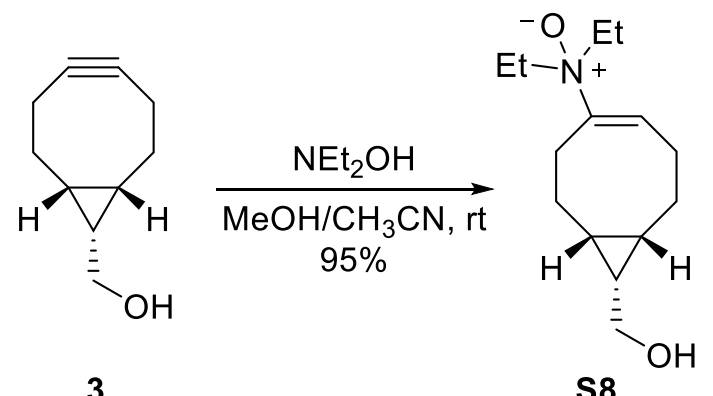

3

S8

\section{(1R,8S,9S,E)- $N, N$-diethyl-9-(hydroxymethyl)bicyclo[6.1.0]non-4-en-4-amine oxide (S8):}

$N, N$-Diethylhydroxylamine $(30.8 \mu \mathrm{L}, 300 \mu \mathrm{mol}, 1.50$ equiv) was added via syringe to a solution of cyclooctyne $3(30.0 \mathrm{mg}, 200 \mu \mathrm{mol}, 1$ equiv) in methanol $(500 \mu \mathrm{L})$ and acetonitrile $(2.0 \mathrm{~mL})$ at room temperature. After $10 \mathrm{~min}$, the reaction mixture was concentrated under reduced pressure. The crude mixture was purified by flash column chromatography on silica gel (eluent: $20 \rightarrow 40 \%$ CMA in chloroform) to provide enamine $N$-oxide $\mathbf{S 8}(45.6 \mathrm{mg}, 95 \%)$ as a clear, colorless oil.

${ }^{1} \mathrm{H}$ NMR $\left(500 \mathrm{MHz}, \mathrm{CD}_{3} \mathrm{OD}, 25{ }^{\circ} \mathrm{C}\right): \delta 6.65(\mathrm{t}, J=8.4 \mathrm{~Hz}, 1 \mathrm{H}), 3.72-3.60(\mathrm{~m}, 2 \mathrm{H}), 3.61-3.42(\mathrm{~m}$, 2H), 3.40-3.29 (m, 2H), $2.63(\mathrm{dt}, J=16.5,5.9 \mathrm{~Hz}, 1 \mathrm{H}), 2.55-2.40(\mathrm{~m}, 2 \mathrm{H}), 2.26-2.12(\mathrm{~m}, 2 \mathrm{H}), 2.15-$ $2.02(\mathrm{~m}, 1 \mathrm{H}), 1.74-1.57(\mathrm{~m}, 2 \mathrm{H}), 1.21(\mathrm{td}, J=7.1,2.5 \mathrm{~Hz}, 6 \mathrm{H}), 1.19-1.10(\mathrm{~m}, 1 \mathrm{H}), 1.11-1.01(\mathrm{~m}$, $2 \mathrm{H})$.

${ }^{13} \mathrm{C}$ NMR $\left(126 \mathrm{MHz}, \mathrm{CD}_{3} \mathrm{OD}, 25{ }^{\circ} \mathrm{C}\right): \delta 148.4,127.5,62.8,62.7,59.7,27.4,25.9,24.9,24.7,22.6$, $20.9,19.5,8.9,8.8$.

FTIR (thin film) $\mathrm{cm}^{-1}$ : 3235 (br), 2986 (m), 2937 (m), 2866 (m), 1461 (m), 1375 (m), 1033 (s).

HRMS (ESI) $(m / z)$ : calc'd for $\mathrm{C}_{14} \mathrm{H}_{26} \mathrm{NO}_{2}[\mathrm{M}+\mathrm{H}]^{+}: 240.1958$, found: 240.1957 .

TLC (50\% CMA in chloroform), Rf: $0.16\left(\mathrm{KMnO}_{4}\right)$.

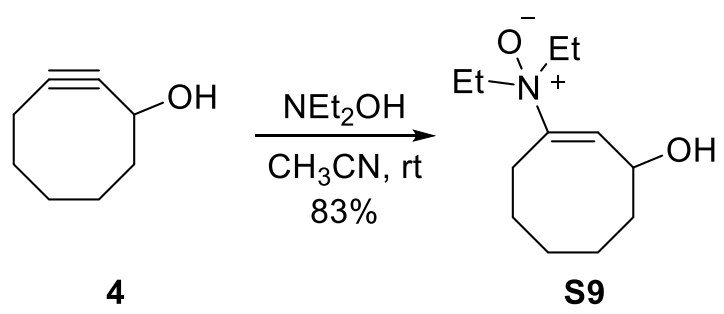

\section{$(E)-N, N$-diethyl-3-hydroxycyclooct-1-en-1-amine oxide (S9):}

$N, N$-Diethylhydroxylamine $(30.8 \mu \mathrm{L}, 300 \mu \mathrm{mol}, 1.50$ equiv) was added via syringe to a solution of cyclooctyne $3(24.8 \mathrm{mg}, 200 \mu \mathrm{mol}, 1$ equiv) in acetonitrile $(2.0 \mathrm{~mL})$ at room temperature. After 5 min, the reaction mixture was concentrated under reduced pressure. The crude mixture was purified by flash column chromatography on silica gel (eluent: $20 \rightarrow 40 \% \mathrm{CMA}$ in chloroform) to provide enamine $N$-oxide $\mathbf{S 9}$ (35.4 $\mathrm{mg}, 83 \%$ ) as a clear, colorless oil. 
${ }^{1} \mathrm{H}$ NMR $\left(500 \mathrm{MHz}, \mathrm{CD}_{3} \mathrm{OD}, 25{ }^{\circ} \mathrm{C}\right): \delta 6.40(\mathrm{~d}, J=7.3 \mathrm{~Hz}, 1 \mathrm{H}), 4.55-4.44(\mathrm{~m}, 1 \mathrm{H}), 3.66-3.55(\mathrm{~m}$, $1 \mathrm{H}), 3.53-3.42(\mathrm{~m}, 1 \mathrm{H}), 3.41-3.31(\mathrm{~m}, 2 \mathrm{H}), 2.58-2.48(\mathrm{~m}, 1 \mathrm{H}), 2.48-2.36(\mathrm{~m}, 1 \mathrm{H}), 2.00-1.92(\mathrm{~m}$, $1 \mathrm{H}), 1.88-1.76(\mathrm{~m}, 1 \mathrm{H}), 1.76-1.43(\mathrm{~m}, 6 \mathrm{H}), 1.31(\mathrm{t}, J=7.1 \mathrm{~Hz}, 3 \mathrm{H}), 1.18(\mathrm{t}, J=7.1 \mathrm{~Hz}, 3 \mathrm{H})$.

${ }^{13} \mathrm{C}$ NMR $\left(126 \mathrm{MHz}, \mathrm{CD}_{3} \mathrm{OD}, 25{ }^{\circ} \mathrm{C}\right): \delta 146.1,132.0,69.9,63.5,61.8,39.0,30.9,27.4,27.0,25.1$, 9.1,9.0.

FTIR (thin film) $\mathrm{cm}^{-1}: 3310$ (br), 2930 (s), 2490 (br), 2065 (w), 1454 (s), 1062 (s), 984 (s).

HRMS (ESI) $(m / z)$ : calc'd for $\mathrm{C}_{12} \mathrm{H}_{24} \mathrm{NO}_{2}[\mathrm{M}+\mathrm{H}]^{+}: 214.1802$, found: 214.1801 .

TLC (50\% CMA in $\left.\mathrm{CHCl}_{3}\right), \mathrm{Rf}: 0.14\left(\mathrm{KMnO}_{4}\right)$.

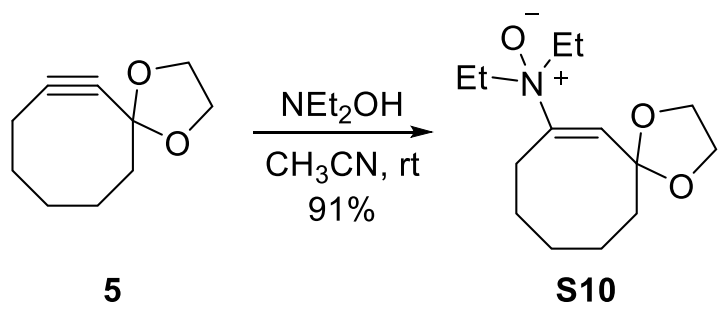

$(E)-N, N$-diethyl-1,4-dioxaspiro[4.7]dodec-6-en-7-amine oxide (S10):

$N, N$-Diethylhydroxylamine $(17.0 \mu \mathrm{L}, 165 \mu \mathrm{mol}, 1.50$ equiv) was added via syringe to a solution of cyclooctyne $7(18.3 \mathrm{mg}, 110 \mu \mathrm{mol}, 1$ equiv) in acetonitrile $(1.0 \mathrm{~mL})$ at room temperature. After 10 min, the reaction mixture was concentrated under reduced pressure. The crude mixture was purified by flash column chromatography on silica gel (eluent: $15 \rightarrow 30 \% \mathrm{CMA}$ in chloroform) to provide enamine $N$-oxide $\mathbf{S 1 0}$ (25.6 $\mathrm{mg}, 91 \%$ ) as a clear thin film.

${ }^{1} \mathrm{H}$ NMR $\left(500 \mathrm{MHz}, \mathrm{CD}_{3} \mathrm{OD}, 25{ }^{\circ} \mathrm{C}\right): \delta 6.67(\mathrm{~s}, 1 \mathrm{H}), 3.99-3.88(\mathrm{~m}, 4 \mathrm{H}), 3.55-3.33(\mathrm{~m}, 4 \mathrm{H}), 2.78(\mathrm{t}, J$ $=6.7 \mathrm{~Hz}, 2 \mathrm{H}), 2.01-1.87(\mathrm{~m}, 2 \mathrm{H}), 1.80-1.61(\mathrm{~m}, 6 \mathrm{H}), 1.21(\mathrm{t}, J=7.1 \mathrm{~Hz}, 6 \mathrm{H})$.

${ }^{13} \mathrm{C}$ NMR $\left(126 \mathrm{MHz}, \mathrm{CD}_{3} \mathrm{OD}, 25{ }^{\circ} \mathrm{C}\right): \delta 148.9,131.7,110.0,65.4,63.1,40.6,29.9,26.0,24.1,23.6$, 9.0.

FTIR (thin film) cm $\mathrm{cm}^{-1}: 3355$ (br), 2933 (m), 1677 (w), 1454 (m), 1081 (s), 1029 (s), 954 (s).

HRMS (ESI) $(m / z)$ : calc'd for $\mathrm{C}_{14} \mathrm{H}_{26} \mathrm{NO}_{3}[\mathrm{M}+\mathrm{H}]^{+}:$256.1907, found: 256.1906.

TLC (50\% CMA in chloroform), $\mathrm{R} f: 0.35\left(\mathrm{KMnO}_{4}\right)$. 


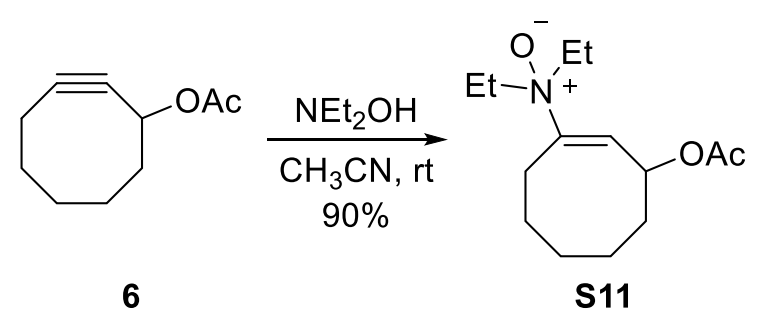

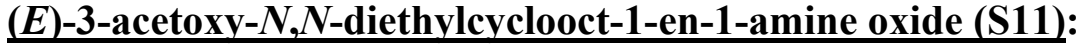

$N, N$-Diethylhydroxylamine $(30.8 \mu \mathrm{L}, 300 \mu \mathrm{mol}, 1.50$ equiv) was added via syringe to a solution of cyclooctyne $8(33.2 \mathrm{mg}, 200 \mu \mathrm{mol}, 1$ equiv) in acetonitrile $(2.0 \mathrm{~mL})$ at room temperature. After 5 min, the reaction mixture was concentrated under reduced pressure. The crude mixture was purified by flash column chromatography on silica gel (eluent: $10 \rightarrow 30 \% \mathrm{CMA}$ in chloroform) to provide enamine $N$-oxide $\mathbf{S 1 1}$ (46.1 mg, 90\%) as a clear, colorless oil.

${ }^{1} \mathrm{H}$ NMR $\left(500 \mathrm{MHz}, \mathrm{CD}_{3} \mathrm{OD}, 25{ }^{\circ} \mathrm{C}\right): \delta 6.40(\mathrm{~d}, J=7.8 \mathrm{~Hz}, 1 \mathrm{H}), 5.47$ (ddd, $\left.J=11.7,7.7,4.8 \mathrm{~Hz}, 1 \mathrm{H}\right)$, $3.62(\mathrm{dq}, J=12.5,7.1 \mathrm{~Hz}, 1 \mathrm{H}), 3.48(\mathrm{dq}, J=12.4,7.2 \mathrm{~Hz}, 1 \mathrm{H}), 3.42-3.28(\mathrm{~m}, 2 \mathrm{H}), 2.58-2.45(\mathrm{~m}$, $2 \mathrm{H}), 2.05(\mathrm{~s}, 3 \mathrm{H}), 2.02-1.93(\mathrm{~m}, 1 \mathrm{H}), 1.93-1.84(\mathrm{~m}, 1 \mathrm{H}), 1.82-1.68(\mathrm{~m}, 3 \mathrm{H}), 1.68-1.60(\mathrm{~m}, 1 \mathrm{H})$, 1.60-1.46 (m, 2H), $1.31(\mathrm{t}, J=7.1 \mathrm{~Hz}, 3 \mathrm{H}), 1.12(\mathrm{t}, J=7.1 \mathrm{~Hz}, 3 \mathrm{H})$.

${ }^{13} \mathrm{C}$ NMR $\left(126 \mathrm{MHz}, \mathrm{CD}_{3} \mathrm{OD}, 25{ }^{\circ} \mathrm{C}\right): \delta 172.2,147.8,128.4,73.2,63.6,62.1,35.3,30.6,27.3,27.3$, 24.6, 21.1, 9.1, 8.8.

FTIR (thin film) $\mathrm{cm}^{-1}$ : 3235 (br), 2933 (w), 1730 (m), 1454 (w), 1368 (w), 1238 (s), 1029 (m).

HRMS (ESI) $(m / z)$ : calc'd for $\mathrm{C}_{14} \mathrm{H}_{26} \mathrm{NO}_{3}[\mathrm{M}+\mathrm{H}]^{+}: 256.1907$, found: 256.1905.

TLC (50\% CMA in chlorofrom), $\mathrm{R} f: 0.16\left(\mathrm{KMnO}_{4}\right)$.

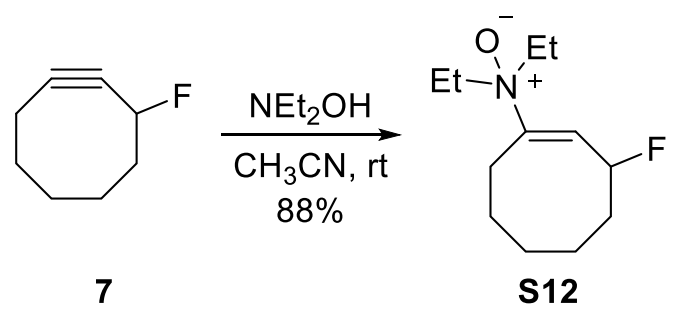

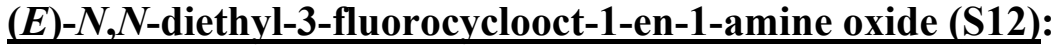

$N, N$-Diethylhydroxylamine $(6.72 \mu \mathrm{L}, 65.4 \mu \mathrm{mol}, 2.00$ equiv) was added via syringe to a solution of cyclooctyne $7(5.5 \mathrm{mg}, 43.6 \mu \mathrm{mol}, 1$ equiv) in acetonitrile $(500 \mu \mathrm{L})$ at room temperature. After 10 min, the reaction mixture was concentrated under reduced pressure. The crude mixture was purified by flash column chromatography on silica gel (eluent: $15 \rightarrow 30 \%$ CMA in chloroform) to provide enamine $N$-oxide $\mathbf{S 1 2}$ (8.3 $\mathrm{mg}, 88 \%$ ) as a clear thin film.

${ }^{1} \mathrm{H}$ NMR (500 MHz, CD $\left.3 \mathrm{OD}, 25{ }^{\circ} \mathrm{C}\right): \delta 6.64(\mathrm{dd}, J=19.8,6.5 \mathrm{~Hz}, 1 \mathrm{H}), 5.47-5.29(\mathrm{~m}, 1 \mathrm{H}), 3.69-3.44$ (m, 2H), 3.43-3.32 (m, 2H), 2.60-2.49 (m, 1H), 2.48-2.36 (m, 1H), 2.20-2.06 (m, 1H), 1.85-1.75 $(\mathrm{m}, 2 \mathrm{H}), 1.73-1.55(\mathrm{~m}, 5 \mathrm{H}), 1.30(\mathrm{t}, J=7.1 \mathrm{~Hz}, 3 \mathrm{H}), 1.17$ (t, $J=7.1 \mathrm{~Hz}, 3 \mathrm{H})$. 
${ }^{13} \mathrm{C}$ NMR $\left(126 \mathrm{MHz}, \mathrm{CD}_{3} \mathrm{OD}, 25{ }^{\circ} \mathrm{C}\right): \delta 147.34(\mathrm{~d}, J=13.4 \mathrm{~Hz}), 128.83(\mathrm{~d}, J=33.4 \mathrm{~Hz}), 92.09(\mathrm{~d}, J$ $=161.6 \mathrm{~Hz}), 63.69,62.13,36.82(\mathrm{~d}, J=21.9 \mathrm{~Hz}), 30.47,26.87,26.60,23.98(\mathrm{~d}, J=12.9 \mathrm{~Hz}), 8.99$, 8.85 .

${ }^{19} \mathrm{~F}$ NMR (471 MHz, $\left.\mathrm{CD}_{3} \mathrm{OD}, 25^{\circ} \mathrm{C}\right): \delta-172.0$.

FTIR (thin film) $\mathrm{cm}^{-1}: 3373$ (br), 2937 (s), 2863 (m), 1595 (m), 1454 (m), 1379 (m), 958 (s).

HRMS (ESI) $(\mathrm{m} / \mathrm{z})$ : calc'd for C12H23FNO [M+H] $]^{+}: 216.1758$, found: 216.1758.

TLC (30\% CMA in chloroform), $\mathrm{R} f: 0.13\left(\mathrm{KMnO}_{4}\right)$.

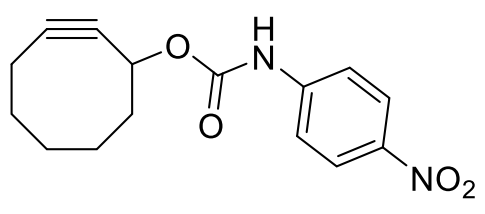

9

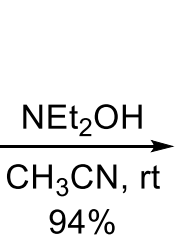

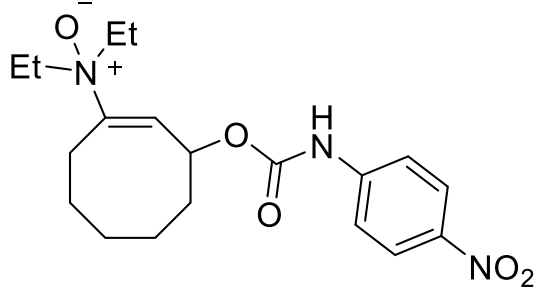

S13

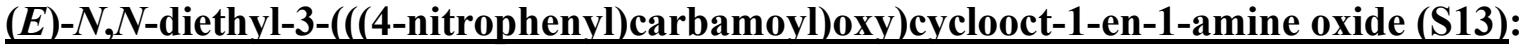

$N, N$-Diethylhydroxylamine $(7.1 \mu \mathrm{L}, 69.2 \mu \mathrm{mol}, 1.50$ equiv) was added via syringe to a solution of cyclooctyne 9 (13.3 mg, $46.1 \mu \mathrm{mol}, 1$ equiv) in acetonitrile $(1.0 \mathrm{~mL})$ at room temperature. After 10 min, the reaction mixture was concentrated under reduced pressure. The crude mixture was purified by flash column chromatography on silica gel (eluent: 30\% CMA in chloroform) to provide enamine $N$-oxide S13 (16.3 mg, 94\%) as a clear thin film.

${ }^{1} \mathrm{H}$ NMR $\left(500 \mathrm{MHz}, \mathrm{CD}_{3} \mathrm{OD}, 25{ }^{\circ} \mathrm{C}\right): \delta 8.16(\mathrm{~d}, J=9.3 \mathrm{~Hz}, 2 \mathrm{H}), 7.63(\mathrm{~d}, J=9.3 \mathrm{~Hz}, 2 \mathrm{H}), 6.53(\mathrm{~d}, J=$ 7.6 Hz, 1H), 5.53 (ddd, $J=11.9,7.7,4.9 \mathrm{~Hz}, 1 \mathrm{H}), 3.69-3.57(\mathrm{~m}, 1 \mathrm{H}), 3.55-3.30(\mathrm{~m}, 3 \mathrm{H}), 2.61-2.49$ $(\mathrm{m}, 2 \mathrm{H}), 2.14-2.03(\mathrm{~m}, 1 \mathrm{H}), 1.94-1.86(\mathrm{~m}, 1 \mathrm{H}), 1.86-1.71(\mathrm{~m}, 3 \mathrm{H}), 1.69-1.49(\mathrm{~m}, 3 \mathrm{H}), 1.33(\mathrm{t}, J=$ $7.1 \mathrm{~Hz}, 3 \mathrm{H}), 1.15$ (t, $J=7.2 \mathrm{~Hz}, 3 \mathrm{H})$.

${ }^{13} \mathrm{C}$ NMR (126 MHz, CD $\left.{ }_{3} \mathrm{OD}, 25{ }^{\circ} \mathrm{C}\right): \delta 154.6,148.0,146.9,143.9,128.4,126.0,119.0,74.1,63.6$, $62.2,35.5,30.6,27.3,27.3,24.6,9.1,8.9$.

FTIR (thin film) $\mathrm{cm}^{-1}: 3198$ (br), 2933 (w), 1726 (w), 1516 (m), 1327 (m), 1223 (s), 1044 (m).

HRMS (ESI) $(m / z)$ : calc'd for $\mathrm{C}_{19} \mathrm{H}_{28} \mathrm{~N}_{3} \mathrm{O}_{5}[\mathrm{M}+\mathrm{H}]^{+}: 378.2023$, found: 378.2021 .

TLC (30\% CMA in chloroform), Rf: $0.26\left(\mathrm{KMnO}_{4}\right)$. 


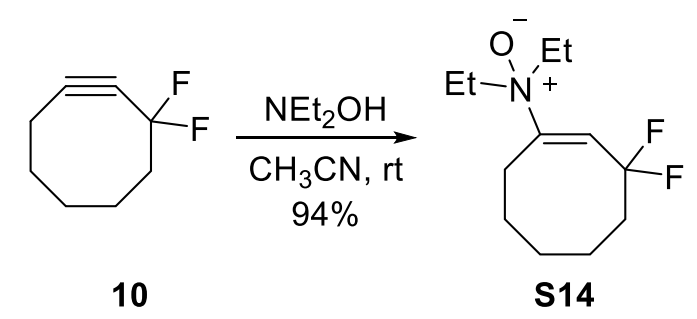

(E)-N,N-diethyl-3,3-difluorocyclooct-1-en-1-amine oxide (S14):

$N, N$-Diethylhydroxylamine $(30.8 \mu \mathrm{L}, 300 \mu \mathrm{mol}, 1.50$ equiv) was added via syringe to a solution of cyclooctyne $\mathbf{1 0}^{3}(28.8 \mathrm{mg}, 200 \mu \mathrm{mol}, 1$ equiv) in acetonitrile $(1.84 \mathrm{~mL})$ at room temperature. After 5 min, the reaction mixture was concentrated under reduced pressure. The crude mixture was purified by flash column chromatography on silica gel (eluent: $10 \rightarrow 30 \% \mathrm{CMA}$ in chloroform) to provide enamine $N$-oxide $\mathbf{S 1 4}$ (43.8 $\mathrm{mg}, 94 \%$ ) as a clear thin film.

${ }^{1} \mathrm{H}$ NMR $\left(500 \mathrm{MHz}, \mathrm{CD}_{3} \mathrm{OD}, 25{ }^{\circ} \mathrm{C}\right): \delta 7.02(\mathrm{t}, J=11.7 \mathrm{~Hz}, 1 \mathrm{H}), 3.61-3.50(\mathrm{~m}, 2 \mathrm{H}), 3.48-3.36(\mathrm{~m}$, $2 \mathrm{H}), 2.73(\mathrm{t}, J=6.9 \mathrm{~Hz}, 2 \mathrm{H}), 2.27(\mathrm{tt}, J=15.6,6.5 \mathrm{~Hz}, 2 \mathrm{H}), 1.85-1.61(\mathrm{~m}, 6 \mathrm{H}), 1.21(\mathrm{t}, J=7.1 \mathrm{~Hz}$, $6 \mathrm{H})$.

${ }^{13} \mathrm{C}$ NMR $\left(126 \mathrm{MHz}, \mathrm{CD}_{3} \mathrm{OD}, 25{ }^{\circ} \mathrm{C}\right): \delta 151.9(\mathrm{t}, J=11.7 \mathrm{~Hz}), 126.8(\mathrm{t}, J=35.0 \mathrm{~Hz}), 123.7(\mathrm{t}, J=$ $233.7 \mathrm{~Hz}), 63.3,38.2,28.3,24.9,24.5,22.2,8.8$.

${ }^{19} \mathrm{~F}$ NMR $\left(471 \mathrm{MHz}, \mathrm{CD}_{3} \mathrm{OD}, 25^{\circ} \mathrm{C}\right): \delta-83.4$.

FTIR (thin film) cm $\mathrm{cm}^{-1}: 3232$ (br), 2937 (m), 1692 (m), 1457 (m), 1316 (m), 988 (s).

HRMS (ESI) $(m / z)$ : calc'd for $\mathrm{C}_{2}{ }_{2} \mathrm{H}_{22} \mathrm{~F}_{2} \mathrm{NO}[\mathrm{M}+\mathrm{H}]^{+}: 234.1664$, found: 234.1664 .

TLC (30\% CMA in chloroform), Rf: $0.059\left(\mathrm{KMnO}_{4}\right)$.

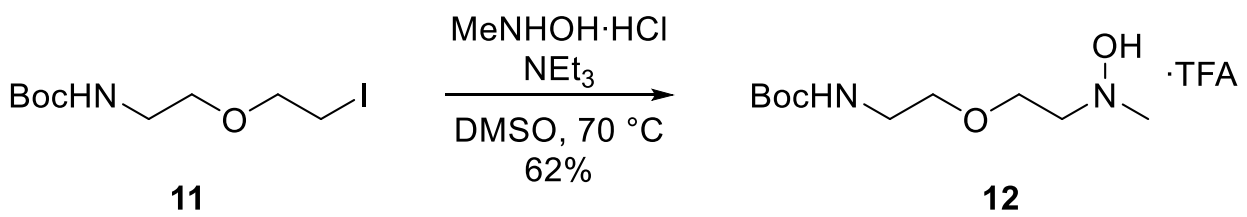

\section{tert-butyl (2-(2-(hydroxy(methyl)amino)ethoxy)ethyl)carbamate (12):}

Triethylamine (1.34 mL, $9.59 \mathrm{mmol}, 4.00$ equiv) was added to a solution of iodoalkane $\mathbf{1 1}^{4}$ (756 $\mathrm{mg}$, $2.40 \mathrm{mmol}, 1$ equiv) and $\mathrm{N}$-methylhydroxylamine hydrochloride (401 $\mathrm{mg}, 4.80 \mathrm{mmol}, 2.00$ equiv) in dimethyl sulfoxide $(2.4 \mathrm{~mL})$ at room temperature. The reaction mixture was then heated to $70{ }^{\circ} \mathrm{C}$. After $1.5 \mathrm{~h}$, the solution was cooled to room temperature, diluted with water, and purified by automated $\mathrm{C}_{18}$ reverse phase column chromatography $\left(30 \mathrm{~g} \mathrm{C}_{18}\right.$ silica gel, $25 \mu \mathrm{m}$ spherical particles, eluent: $\mathrm{H}_{2} \mathrm{O}+0.1 \%$ TFA (2 CV), gradient $0 \rightarrow 100 \% \mathrm{CH}_{3} \mathrm{CN} / \mathrm{H}_{2} \mathrm{O}+0.1 \%$ TFA $(10$ to $15 \mathrm{CV})$ ) to provide hydroxylamine $12(348 \mathrm{mg}, 62 \%)$ as a white solid.

${ }^{1} \mathrm{H}$ NMR $\left(500 \mathrm{MHz} \mathrm{CDCl}_{3}, 25^{\circ} \mathrm{C}\right) \delta 3.82(\mathrm{ddd}, J=11.1,7.3,3.9 \mathrm{~Hz}, 1 \mathrm{H}), 3.63(\mathrm{dt}, J=11.0,4.2 \mathrm{~Hz}$, $1 \mathrm{H}), 3.52-3.35(\mathrm{~m}, 4 \mathrm{H}), 3.32-3.18(\mathrm{~m}, 2 \mathrm{H}), 3.07(\mathrm{~s}, 3 \mathrm{H}), 1.38(\mathrm{~s}, 9 \mathrm{H})$. 
${ }^{13} \mathrm{C}$ NMR $\left(126 \mathrm{MHz}, \mathrm{CDCl}_{3}, 25{ }^{\circ} \mathrm{C}\right) \delta 164.1(\mathrm{q}, J=37.5 \mathrm{~Hz}), 156.7,116.5(\mathrm{q}, J=289.2 \mathrm{~Hz}), 79.5$, $70.9,63.6,60.2,46.5,40.4,28.5$.

${ }^{19} \mathrm{~F}$ NMR $\left(471 \mathrm{MHz}, \mathrm{CDCl}_{3}, 25^{\circ} \mathrm{C}\right) \delta-75.47$.

FTIR (thin film) cm $\mathrm{cm}^{-1}: 3351$ (br), 2945 (w), 2900 (w), 2236 (s), 1361 (m), 1290 (m), 1185 (s), 1129 (s), $1085(\mathrm{~s})$.

HRMS (ESI) $(m / z)$ : calc'd for $\mathrm{C}_{10} \mathrm{H}_{23} \mathrm{~N}_{2} \mathrm{O}_{4}[\mathrm{M}+\mathrm{H}]^{+}:$235.1652, found: 235.1650 .

TLC (40\% CMA in chloroform), Rf: $0.58\left(\mathrm{I}_{2}\right)$.<smiles>CN(C)c1ccc2c(c1)Oc1cc(N(C)C)ccc1C21OC(=O)c2ccc(C(=O)O)cc21</smiles>

6-TAMRA

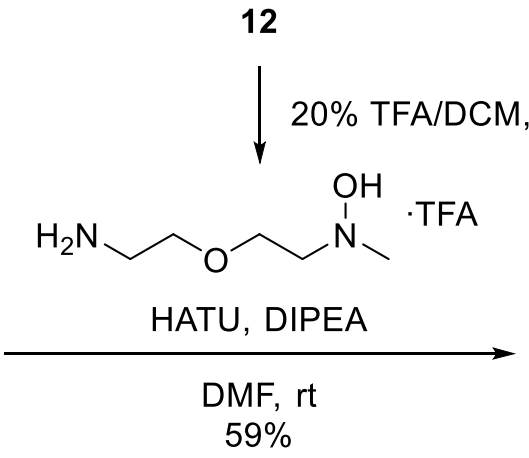

$59 \%$

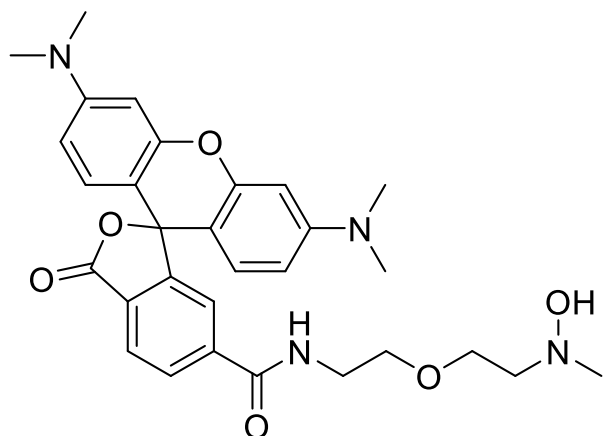

13

3',6'-bis(dimethylamino)-N-(2-(2-(hydroxy(methyl)amino)ethoxy)ethyl)-3-oxo-3Hspiro[isobenzofuran-1,9'-xanthene]-6-carboxamide (13):

$N, N$-Diisopropylethylamine (DIPEA, $49.2 \mu \mathrm{L}, 282 \mu \mathrm{mol}, 4.00$ equiv) was added to a solution of 6 carboxytetramethylrhodamine (6-TAMRA, $30.4 \quad \mathrm{mg}, \quad 70.6 \mu \mathrm{mol}, 1$ equiv) and 1 [bis(dimethylamino)methylene]-1H-1,2,3-triazolo[4,5-b]pyridinium 3-oxid hexafluorophosphate (HATU, $29.5 \mathrm{mg}, 77.7 \mu \mathrm{mol}, 1.10$ equiv) in $N, N$-dimethylformamide (DMF, $700 \mu \mathrm{L}$ ) at room temperature. In a separate vial, trifluoroacetic acid $(100 \mu \mathrm{L})$ was added to a solution of hydroxylamine $12(61.5 \mathrm{mg}, 177 \mu \mathrm{mol}, 2.50$ equiv) in dichloromethane (400 $\mu \mathrm{L})$. The resulting solution was stirred at room temperature for $1 \mathrm{~h}$ then concentrated under reduced pressure. The resulting residue was dissolved in $N, N$-dimethylformamide $(500 \mu \mathrm{L})$ then added to the reaction mixture containing 6-TAMRA using a pipette. An additional portion of $N, N$-dimethylformamide (200 $\mu \mathrm{L})$ was used to quantitatively transfer the hydroxylamine solution to the reaction mixture. The reaction mixture was stirred at room temperature for $4 \mathrm{~h}$. An additional portion of HATU (29.5 mg, $77.7 \mu \mathrm{mol}, 1.10$ equiv) and DIPEA $(49.2 \mu \mathrm{L}, 282 \mu \mathrm{mol}, 4.00$ equiv) was added to the reaction mixture. The solution was then stirred for another $2.5 \mathrm{~h}$. The resulting mixture was diluted with water and purified by automated $\mathrm{C}_{18}$ reverse phase column chromatography $\left(30 \mathrm{~g} \mathrm{C}_{18}\right.$ silica gel, $25 \mu \mathrm{m}$ spherical particles, eluent: $\mathrm{H}_{2} \mathrm{O}+0.1 \%$ TFA $(2 \mathrm{CV})$, gradient $0 \rightarrow 100 \% \mathrm{CH}_{3} \mathrm{CN} / \mathrm{H}_{2} \mathrm{O}+0.1 \%$ TFA $(10$ to $15 \mathrm{CV})$ ) and flash column chromatography on silica gel (eluent: $70 \% \mathrm{CMA}$ in $\mathrm{CHCl}_{3}$ ) to provide TAMRA-hydroxylamine 13 (22.8 $\mathrm{mg}, 59 \%)$ as a violet solid. 
${ }^{1} \mathrm{H}$ NMR $\left(500 \mathrm{MHz}, \mathrm{D}_{2} \mathrm{O}, 25^{\circ} \mathrm{C}\right) \delta 7.99(\mathrm{~d}, J=8.3 \mathrm{~Hz}, 1 \mathrm{H}), 7.87(\mathrm{~d}, J=8.1 \mathrm{~Hz}, 1 \mathrm{H}), 7.67-7.59(\mathrm{~m}$, $1 \mathrm{H}), 7.07$ (d, $J=9.5 \mathrm{~Hz}, 2 \mathrm{H}), 6.73(\mathrm{dd}, J=9.5,2.4 \mathrm{~Hz}, 2 \mathrm{H}), 6.41(\mathrm{~d}, J=2.3 \mathrm{~Hz}, 2 \mathrm{H}), 3.84-3.63$ (m, $4 \mathrm{H}), 3.53(\mathrm{t}, J=5.4 \mathrm{~Hz}, 2 \mathrm{H}), 3.11-2.89(\mathrm{~m}, 14 \mathrm{H}), 2.71(\mathrm{~s}, 3 \mathrm{H})$.

${ }^{13} \mathrm{C}$ NMR $\left(126 \mathrm{MHz}, \mathrm{D}_{2} \mathrm{O}, 25{ }^{\circ} \mathrm{C}\right) \delta 173.2,168.0,157.6,156.7,156.6,143.0,133.5,130.9,130.6$, $129.1,128.9,128.1,113.6,112.7,96.1,68.8,66.6,60.2,47.5,34.0,39.7$.

FTIR (thin film) cm $\mathrm{cm}^{-1}: 3280$ (br), 2926 (w), 1648 (w) 1595 (s), 1491 (m), 1409 (m), 1349 (m), 1189 $(\mathrm{m})$.

HRMS (ESI) $(m / z)$ : calc'd for $\mathrm{C}_{30} \mathrm{H}_{35} \mathrm{~N}_{4} \mathrm{O}_{6}[\mathrm{M}+\mathrm{H}]^{+}: 547.2551$, found: 547.2544 .

TLC (100\% CMA), Rf: 0.37 (visual).
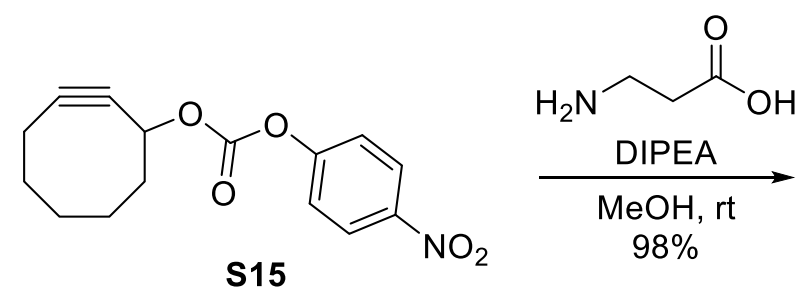

\section{3-(((cyclooct-2-yn-1-yloxy)carbonyl)amino)propanoic acid (S16):}

3-aminopropanoic acid (18.5 mg, $207 \mu \mathrm{mol}, 1.20$ equiv) was added to a solution of carbonate $\mathbf{S 1 5}^{5}$ $(50.0 \mathrm{mg}, 173 \mu \mathrm{mol}, 1$ equiv) in methanol $(2.0 \mathrm{~mL})$ at room temperature. $N, N$-Diisopropylethylamine $(90.3 \mu \mathrm{L}, 519 \mu \mathrm{mol}, 3.00$ equiv) was then added to the solution. After $1 \mathrm{~h}$, the reaction mixture was concentrated under reduced pressure. The crude mixture was purified by flash column chromatography on silica gel (eluent: hexanes/ethyl acetate/acetic acid, $\mathrm{v} / \mathrm{v} / \mathrm{v}=65: 30: 5$ ) to provide carbamate S16 (40.4 mg, 98\%) as a clear, colorless oil.

${ }^{1} \mathrm{H}$ NMR $\left(500 \mathrm{MHz}, \mathrm{CD}_{3} \mathrm{OD}, 25^{\circ} \mathrm{C}\right): \delta 5.23-5.10(\mathrm{~m}, 1 \mathrm{H}), 3.37-3.31(\mathrm{~m}, 2 \mathrm{H}), 2.49(\mathrm{t}, J=6.9 \mathrm{~Hz}$, $3 \mathrm{H}), 2.29-2.20(\mathrm{~m}, 1 \mathrm{H}), 2.21-2.07(\mathrm{~m}, 2 \mathrm{H}), 2.03-1.94(\mathrm{~m}, 1 \mathrm{H}), 1.95-1.85(\mathrm{~m}, 2 \mathrm{H}), 1.86-1.75(\mathrm{~m}$, $1 \mathrm{H}), 1.73-1.60(\mathrm{~m}, 2 \mathrm{H}), 1.60-1.50(\mathrm{~m}, 1 \mathrm{H})$.

${ }^{13} \mathrm{C}$ NMR (126 MHz, $\left.\mathrm{CD}_{3} \mathrm{OD}, 25{ }^{\circ} \mathrm{C}\right): \delta 175.5,158.2,102.0,92.4,68.2,43.1,37.9,35.5,35.3,31.0$, 27.4, 21.3.

FTIR (thin film) $\mathrm{cm}^{-1}: 2930$ (m), 1700 (s), 1528 (m), 1252 (m), 1137 (w).

HRMS (ESI) $(m / z)$ : calc'd for $\mathrm{C}_{12} \mathrm{H}_{18} \mathrm{NO}_{4}[\mathrm{M}+\mathrm{H}]^{+}: 240.1230$, found: 240.1229 .

TLC (5\% methanol in dichloromethane $+0.1 \%$ acetic acid), Rf: $0.19\left(\mathrm{I}_{2}\right)$. 


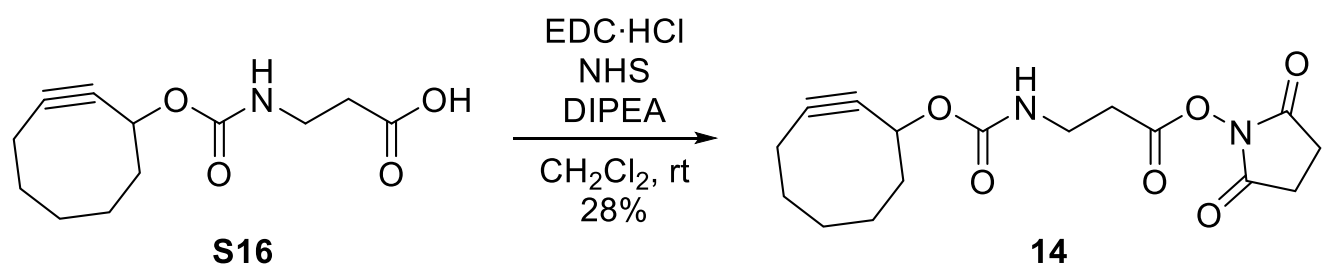

2,5-dioxopyrrolidin-1-yl 3-(((cyclooct-2-yn-1-yloxy)carbonyl)amino)propanoate (14):

$N$-Hydroxysuccinimide (NHS, $22.0 \mathrm{mg}, 191 \mu \mathrm{mol}, 2.50$ equiv), ethylcarbodiimide hydrochloride (EDC $\cdot \mathrm{HCl}, 36.7 \mathrm{mg}, 191 \mu \mathrm{mol}, 2.50$ equiv), and $N, N$-diisopropylethylamine $(53.3 \mu \mathrm{L}, 306 \mu \mathrm{mol}, 4.00$ equiv) were sequentially added to a solution of carboxylic acid $\mathbf{S 1 6}$ (18.3 $\mathrm{mg}, 76.5 \mu \mathrm{mol}, 1$ equiv) in dichloromethane $(1.0 \mathrm{~mL})$ at room temperature. After $12 \mathrm{~h}$, additional NHS $(44.0 \mathrm{mg}, 382 \mu \mathrm{mol}, 5.00$ equiv) and $\mathrm{EDC} \cdot \mathrm{HCl}(73.4 \mathrm{mg}, 382 \mu \mathrm{mol}, 5.00$ equiv) were added to the reaction mixture. After $4 \mathrm{~h}$, the reaction mixture was concentrated under reduced pressure. The crude mixture was purified by flash column chromatography on silica gel (eluent: 30\% acetone in hexanes) to provide NHS-ester 14 (7.1 $\mathrm{mg}, 28 \%)$ as a clear, colorless oil.

${ }^{1} \mathrm{H}$ NMR $\left(500 \mathrm{MHz}, \mathrm{CDCl}_{3}, 2{ }^{\circ} \mathrm{C}\right): \delta$ 5.45-5.19 (m, 2H), 3.63-3.47 (m, 2H), 2.94-2.67 (m, 6H), 2.33-2.20 (m, 1H), 2.21-2.08 (m, 2H), 2.04-1.94 (m, 1H), 1.95-1.81 (m, 2H), 1.81-1.71 (m, 1H), $1.70-1.58(\mathrm{~m}, 2 \mathrm{H}), 1.58-1.45(\mathrm{~m}, 2 \mathrm{H})$.

${ }^{13} \mathrm{C} \mathrm{NMR}\left(126 \mathrm{MHz}, \mathrm{CDCl}_{3}, 25{ }^{\circ} \mathrm{C}\right): \delta 169.2,167.7,155.7,101.9,91.1,67.6,42.0,36.6,34.4,32.3$, 29.8, 26.4, 25.8, 20.9.

FTIR (thin film) $\mathrm{cm}^{-1}: 3358(\mathrm{w}), 2930$ (w), $1782(\mathrm{w}), 1733$ (s), 1517 (m), 1245 (m), 1200 (s)

HRMS (ESI) $(m / z)$ : calc'd for $\mathrm{C}_{16} \mathrm{H}_{21} \mathrm{~N}_{2} \mathrm{O}_{6}[\mathrm{M}+\mathrm{H}]^{+}: 337.1394$, found: 337.1391 .

TLC (50\% ethyl acetate in hexanes), Rf: $0.25\left(\mathrm{I}_{2}\right)$.

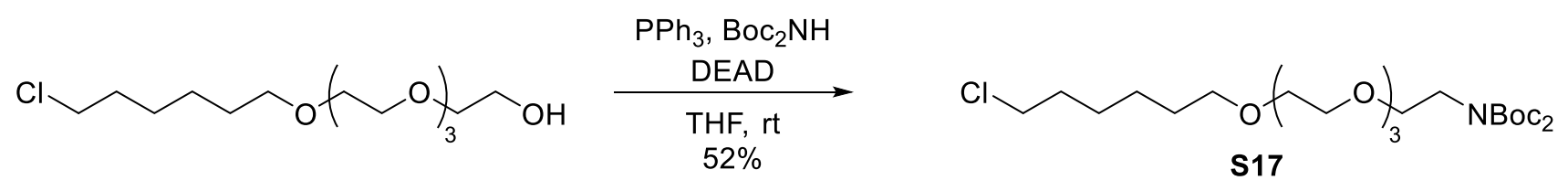

\section{Di-tert-butyl (18-chloro-3,6,9,12-tetraoxaoctadecyl)iminodicarbonate (S17):}

Diethyl azodicarboxylate (DEAD, 40\% in toluene, $233 \mu \mathrm{L}, 512 \mu \mathrm{mol}, 2.00$ equiv) was added dropwise via syringe to a solution of 18-chloro-3,6,9,12-tetraoxaoctadecan-1-ol $(80.0 \mathrm{mg}, 256 \mu \mathrm{mol}$, 1 equiv), triphenylphosphine (131 mg, $512 \mu \mathrm{mol}, 2.00$ equiv), and di-tert-butyl iminodicarbonate (111 mg, $512 \mu \mathrm{mol}, 2.00$ equiv) in tetrahydrofuran $(5 \mathrm{~mL}$ ) at room temperature. After $2.5 \mathrm{~h}$, the reaction was concentrated under reduced pressure. The crude mixture was purified by flash column chromatography on silica gel (eluent: $20 \%$ acetone in hexanes) to provide chloroalkane $\mathbf{S 1 7}$ (67.7 $\mathrm{mg}$, $52 \%$ ) as a colorless oil.

${ }^{1} \mathrm{H}$ NMR $\left(500 \mathrm{MHz}, \mathrm{CDCl}_{3}\right) \delta 3.74(\mathrm{t}, J=6.3 \mathrm{~Hz}, 2 \mathrm{H}), 3.63-3.51(\mathrm{~m}, 14 \mathrm{H}), 3.48(\mathrm{t}, J=6.7 \mathrm{~Hz}, 2 \mathrm{H})$, $3.41(\mathrm{t}, J=6.6 \mathrm{~Hz}, 2 \mathrm{H}), 1.76-1.68(\mathrm{~m}, 2 \mathrm{H}), 1.59-1.50(\mathrm{~m}, 2 \mathrm{H}), 1.45(\mathrm{~s}, 18 \mathrm{H}), 1.43-1.28(\mathrm{~m}, 4 \mathrm{H})$. 
${ }^{13} \mathrm{C} \mathrm{NMR}\left(126 \mathrm{MHz}, \mathrm{CDCl}_{3}\right) \delta 152.8,82.4,71.4,70.8,70.8,70.7,70.7,70.4,70.2,69.4,45.3,45.2$, $32.7,29.6,28.2,26.8,25.6$.

FTIR (thin film) $\mathrm{cm}^{-1}$ : 2863 (w), 1696 (m), 1349 (m), 1118 (s), 854 (w).

HRMS (ESI) $(m / z)$ : calc'd for $\mathrm{C}_{24} \mathrm{H}_{46} \mathrm{ClNNaO}_{8}[\mathrm{M}+\mathrm{Na}]^{+}:$534.2804, found: 534.2811.

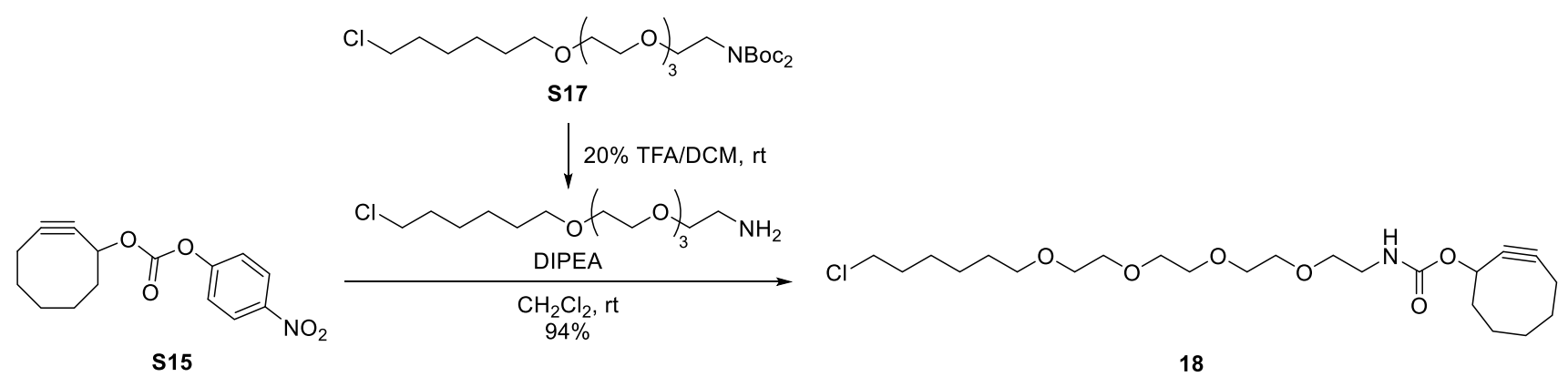

Cyclooct-2-yn-1-yl (18-chloro-3,6,9,12-tetraoxaoctadecyl)carbamate (18):

Trifluoroacetic acid (TFA, $400 \mu \mathrm{L}$ ) was added via syringe to a solution of chloroalkane $\mathbf{S 1 7}$ (38.9 $\mathrm{mg}, 76.0 \mu \mathrm{mol}, 1.10$ equiv) in dichloromethane $(1.60 \mathrm{~mL})$ at room temperature. After $30 \mathrm{~min}$, the reaction was concentrated under reduced pressure then diluted with methanol (1 mL). N,NDiisopropylethylamine (DIPEA, 36.1 $\mu \mathrm{L}, 207 \mu \mathrm{mol}, 3.00$ equiv) and nitrophenyl carbonate S15 (20.0 mg, $69.1 \mu \mathrm{mol}, 1$ equiv) were sequentially added to the solution at room temperature. An additional portion of DIPEA $(12.0 \mu \mathrm{L}, 69.1 \mu \mathrm{mol}, 1.00$ equiv) was added every hour and monitored by TLC until the reaction reached completion. After $4 \mathrm{~h}$, the reaction mixture was purified by flash column chromatography on silica gel (eluent: 60\% ethyl acetate in hexanes) to provide cyclooctyne 18 (30.6 $\mathrm{mg}, 94 \%)$ as a colorless oil.

${ }^{1} \mathrm{H}$ NMR $\left(500 \mathrm{MHz}, \mathrm{CDCl}_{3}\right) \delta 5.38-5.15(\mathrm{~m}, 2 \mathrm{H}), 3.68-3.55(\mathrm{~m}, 12 \mathrm{H}), 3.54-3.48(\mathrm{~m}, 2 \mathrm{H}), 3.44(\mathrm{t}, J$ $=6.6 \mathrm{~Hz}, 2 \mathrm{H}), 3.37-3.29(\mathrm{~m}, 2 \mathrm{H}), 2.30-2.20(\mathrm{~m}, 1 \mathrm{H}), 2.20-2.08(\mathrm{~m}, 2 \mathrm{H}), 2.04-1.94(\mathrm{~m}, 1 \mathrm{H}), 1.95-$ $1.81(\mathrm{~m}, 2 \mathrm{H}), 1.81-1.69(\mathrm{~m}, 4 \mathrm{H}), 1.68-1.62(\mathrm{~m}, 2 \mathrm{H}), 1.61-1.47$ (m, 4H), 1.47-1.39 (m, 2H), 1.39$1.31(\mathrm{~m}, 2 \mathrm{H})$.

${ }^{13} \mathrm{C} \mathrm{NMR}\left(126 \mathrm{MHz}, \mathrm{CDCl}_{3}\right) \delta 155.9,101.6,91.4,71.5,70.9,70.9,70.8,70.8,70.5,70.4,70.3,67.2$, $45.3,42.2,41.0,34.4,32.8,29.9,29.7,26.9,26.4,25.7,20.9$.

FTIR (thin film) cm $\mathrm{cm}^{-1}: 2930$ (m), 2859 (m), 1722 (s), 1521 (w), 1249 (m), 1115 (s).

HRMS (ESI) $(m / z)$ : calc'd for $\mathrm{C}_{23} \mathrm{H}_{41} \mathrm{ClNO}_{6}[\mathrm{M}+\mathrm{H}]^{+}:$462.2617, found: 462.2609 . 


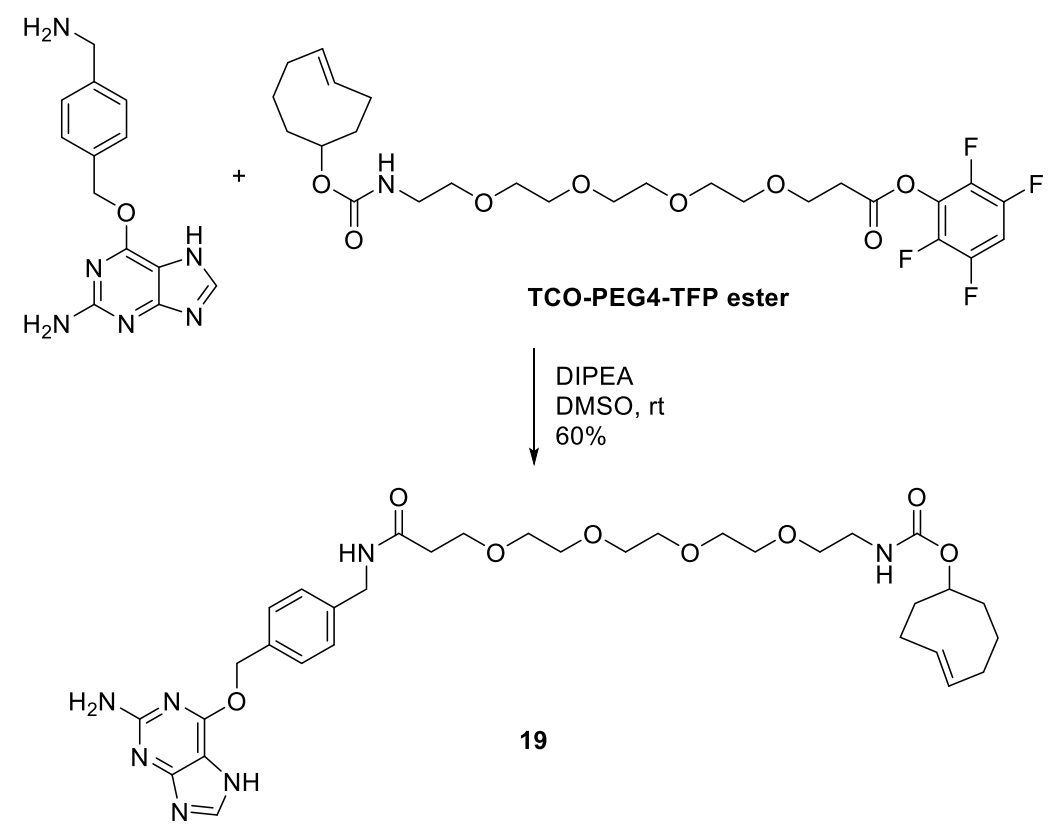

(E)-cyclooct-4-en-1-yl (1-(4-(((2-amino-7H-purin-6-yl)oxy)methyl)phenyl)-3-oxo-6,9,12,15tetraoxa-2-azaheptadecan-17-yl)carbamate (19):

6-((4-(aminomethyl)benzyl)oxy)-7H-purin-2-amine (7.2 $\mathrm{mg}, 26.5 \mu \mathrm{mol}, 1.20$ equiv) and $\mathrm{N}, \mathrm{N}$ diisopropylethylamine $(7.7 \mu \mathrm{L}, 44.2 \mu \mathrm{mol}, 2.00$ equiv) were sequentially added to a solution of TCO-PEG4-TFP ester (Click Chemistry Tools 1398-25, $12.5 \mathrm{mg}, 22.1 \mu \mathrm{mol}, 1$ equiv) in dimethylsulfoxide $(221 \mu \mathrm{L})$ at room temperature. After $2 \mathrm{~h}$, the resulting mixture was diluted with water and purified by automated $\mathrm{C}_{18}$ reverse phase column chromatography $\left(30 \mathrm{~g} \mathrm{C}_{18}\right.$ silica gel, 25 $\mu \mathrm{m}$ spherical particles, eluent: $\mathrm{H}_{2} \mathrm{O}+0.1 \%$ TFA $\left(2 \mathrm{CV}\right.$ ), gradient $0 \rightarrow 100 \% \mathrm{CH}_{3} \mathrm{CN} / \mathrm{H}_{2} \mathrm{O}+0.1 \%$ TFA $(10$ to $15 \mathrm{CV}))$ to provide TCO-benzylguanine $19(8.9 \mathrm{mg}, 60 \%)$ as a clear thin film.

${ }^{1} \mathrm{H}$ NMR $\left(500 \mathrm{MHz}, \mathrm{CD}_{3} \mathrm{OD}\right) \delta 8.39(\mathrm{~s}, 1 \mathrm{H}), 7.52(\mathrm{~d}, J=7.8 \mathrm{~Hz}, 2 \mathrm{H}), 7.35(\mathrm{~d}, J=7.9 \mathrm{~Hz}, 2 \mathrm{H}), 5.66$ $(\mathrm{s}, 2 \mathrm{H}), 5.57(\mathrm{ddd}, J=15.2,10.1,4.5 \mathrm{~Hz}, 1 \mathrm{H}), 5.49-5.39(\mathrm{~m}, 1 \mathrm{H}), 4.41(\mathrm{~s}, 2 \mathrm{H}), 4.34-4.20(\mathrm{~m}, 1 \mathrm{H})$, $3.75(\mathrm{t}, J=6.0 \mathrm{~Hz}, 2 \mathrm{H}), 3.65-3.53(\mathrm{~m}, 12 \mathrm{H}), 3.47(\mathrm{t}, J=5.6 \mathrm{~Hz}, 2 \mathrm{H}), 3.22(\mathrm{t}, J=5.6 \mathrm{~Hz}, 2 \mathrm{H}), 2.50(\mathrm{t}$, $J=6.0 \mathrm{~Hz}, 2 \mathrm{H}), 2.37-2.23(\mathrm{~m}, 3 \mathrm{H}), 2.01-1.82(\mathrm{~m}, 4 \mathrm{H}), 1.75-1.64(\mathrm{~m}, 2 \mathrm{H}), 1.61-1.51(\mathrm{~m}, 1 \mathrm{H})$.

${ }^{13} \mathrm{C}$ NMR $\left(126 \mathrm{MHz}, \mathrm{CD}_{3} \mathrm{OD}\right) \delta 174.2,161.3,161.3,161.0,158.0,153.5,144.2,141.2,136.2,135.3$, $133.9,130.4,128.9,81.9,71.7,71.7,71.6,71.6,71.5,71.4,71.2,71.2,68.4,43.9,42.4,41.7,39.8$, $37.9,35.3,33.6,32.3$.

FTIR (thin film) $\mathrm{cm}^{-1}: 3317$ (br), 2930 (w), 2863 (w), 1666 (s), 1621 (s), 1446 (m), 1200 (s), 1137 (s).

HRMS (ESI) $(m / z)$ : calc'd for $\mathrm{C}_{33} \mathrm{H}_{48} \mathrm{~N}_{7} \mathrm{O}_{8}[\mathrm{M}+\mathrm{H}]^{+}:$670.3559, found: 670.3559 . 


\section{Kinetics Studies}

All kinetics experiments were carried out at room temperature in $\mathrm{CD}_{3} \mathrm{CN}$ or $50 \% \mathrm{H}_{2} \mathrm{O} / \mathrm{CD}_{3} \mathrm{CN}$. Reactions were monitored via NMR spectroscopy using an internal standard. Second order kinetics were performed by combining cyclooctynes and $N, N$-diethylhydroxylamine in a 1:1 ratio. See the following table for experimental conditions for each cyclooctyne. The reported errors for rate constants are based on the standard deviation of the mean for experiments performed in triplicate.

\begin{tabular}{|c|c|c|c|c|}
\hline Compound & Method & Internal standard & Concentration & $k_{2}\left(\mathrm{M}^{-1} \mathrm{~s}^{-1}\right)$ \\
\hline $\mathbf{2}$ & ${ }^{1} \mathrm{H}$ NMR & $1,3,5$-trimethoxybenzene & $7.1 \mathrm{mM}$ & $0.0325 \pm$ \\
& & & 0.0004 \\
\hline $\mathbf{3}$ & ${ }^{1} \mathrm{H}$ NMR & benzotrifluoride & $5.6 \mathrm{mM}$ & $0.217 \pm 0.006$ \\
\hline $\mathbf{4}$ & ${ }^{1} \mathrm{H}$ NMR & benzotrifluoride & $3.3 \mathrm{mM}$ & $1.19 \pm 0.15$ \\
\hline $\mathbf{5}$ & ${ }^{1} \mathrm{H}$ NMR & $1,3,5$-trimethoxybenzene & $6.5 \mathrm{mM}$ & $1.20 \pm 0.09$ \\
\hline $\mathbf{6}$ & ${ }^{1} \mathrm{H}$ NMR & benzotrifluoride & $5.0 \mathrm{mM}$ & $2.13 \pm 0.03$ \\
\hline $\mathbf{7}$ & ${ }^{19} \mathrm{~F} \mathrm{NMR}$ & benzotrifluoride & $8.0 \mathrm{mM}$ & $2.71 \pm 0.43$ \\
\hline $\mathbf{8}$ & ${ }^{1} \mathrm{H}$ NMR & 1,3,5-trimethoxybenzene & $7.4 \mathrm{mM}$ & $2.77 \pm 0.13$ \\
\hline $\mathbf{9}$ & ${ }^{1} \mathrm{H}$ NMR & benzotrifluoride & $5.0 \mathrm{mM}$ & $3.87 \pm 0.55$ \\
\hline $\mathbf{5}$ & ${ }^{19} \mathrm{~F}$ NMR & benzotrifluoride & $10 \mathrm{mM}$ & $1.45 \pm 0.40$ \\
\hline
\end{tabular}

$*$ Kinetics studies were performed in $50 \% \mathrm{H}_{2} \mathrm{O} / \mathrm{CD}_{3} \mathrm{CN}$.

A
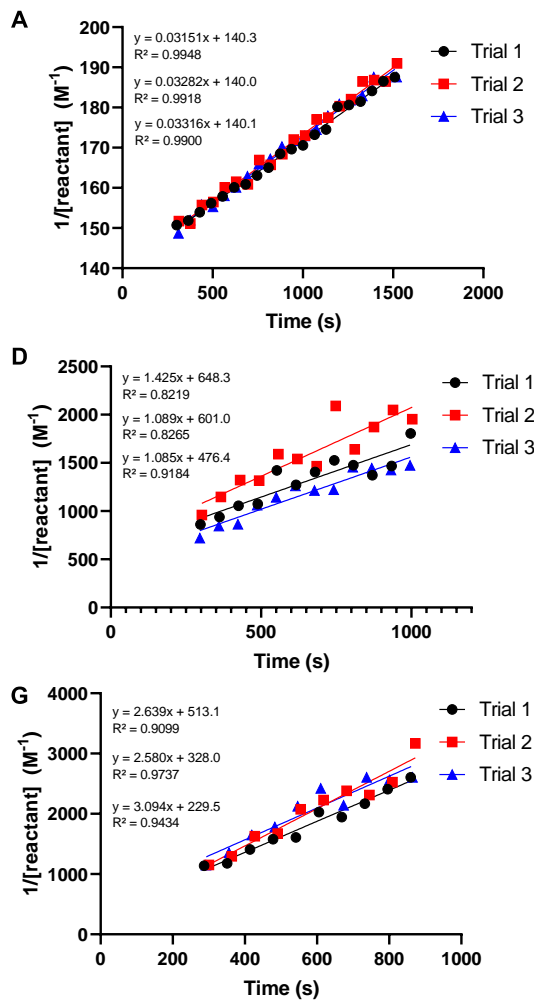
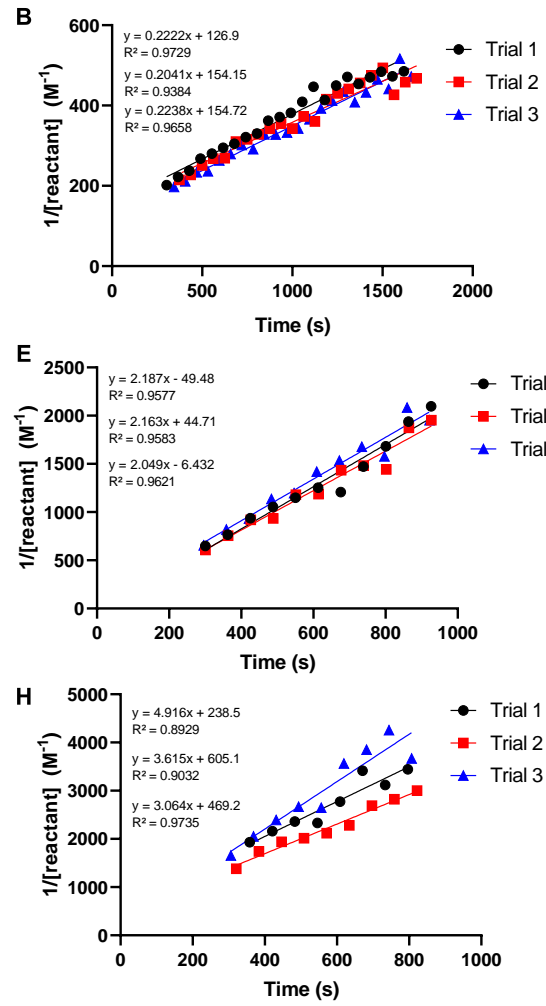
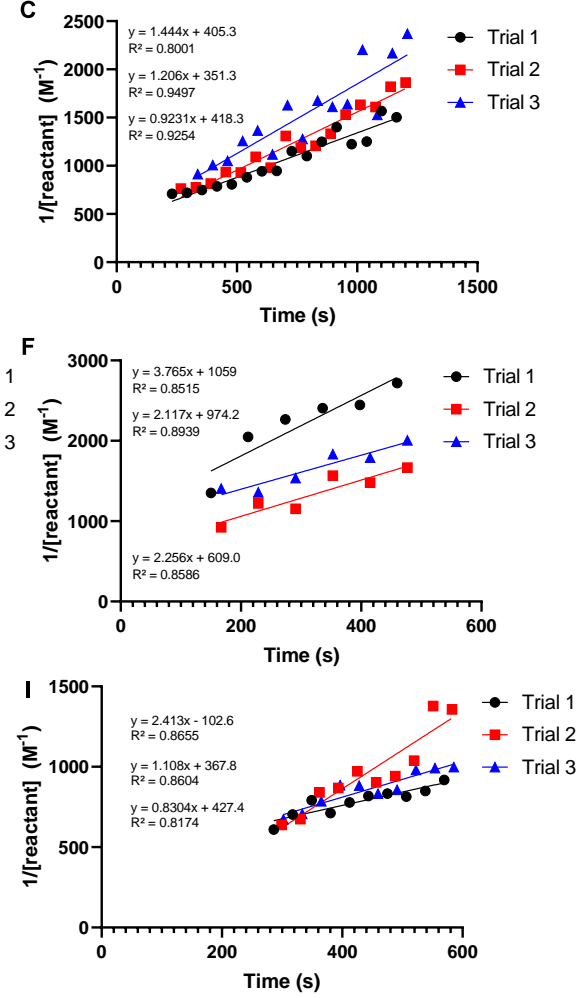

Figure S1. Reaction plots used to calculate second order rate constants for the reactions between $N, N$-diethylhydroxylamine and cyclooctynes A) 2, B) 3, C) 4, D) 5, E) 6, F) 7, G) 8, and H) 9 in $\mathrm{CD}_{3} \mathrm{CN}$ or I) 7 in $50 \% \mathrm{H}_{2} \mathrm{O} / \mathrm{CD}_{3} \mathrm{CN}$. Each panel shows independent experiments done in triplicate. 
The second order rate constant for difluorocyclooctyne 10 in $\mathrm{CD}_{3} \mathrm{CN}$ was determined using a competition experiment with carbamate 9. $N, N$-diethylhydroxylamine ( 1 equiv; $1.9 \mathrm{mM}$ final concentration) was added to a solution containing a 1:4 ratio of difluorocyclooctyne $\mathbf{1 0}$ (5 equiv; 9.5 $\mathrm{mM}$ final concentration) and cyclooctyne carbamate 9 (20 equiv; $38 \mathrm{mM}$ final concentration) in $\mathrm{CD}_{3} \mathrm{CN}$ at room temperature (Figure $\mathrm{S} 2 \mathrm{~A}$ ). The solution was transferred to an NMR tube and the product ratio (S14:S13) was determined by ${ }^{1} \mathrm{H}$ NMR spectroscopy using 1,3,5-trimethoxylbenzene as an internal standard. The second order rate constant $\left(k_{2}\right)$ of difluorocyclooctyne $\mathbf{1 0}$ was calculated by multiplying the observed product ratio with the second order rate constant $\left(k_{2}\right)$ of carbamate 9 to give $83.6 \pm 14.9 \mathrm{M}^{-1} \mathrm{~s}^{-1}$. The reported error for the rate constant is the standard deviation of the mean for experiments performed in triplicate.

In $50 \% \quad \mathrm{H}_{2} \mathrm{O} / \mathrm{CD}_{3} \mathrm{CN}(\mathrm{v} / \mathrm{v})$, the hydroamination between difluorocyclooctyne $\mathbf{1 0}$ and $N, N$ diethylhydroxylamine was completed within $5 \mathrm{~min}$ (Figure S2B). The second order rate constant for difluorocyclooctyne 10 in $50 \% \mathrm{H}_{2} \mathrm{O} / \mathrm{CD}_{3} \mathrm{CN}(\mathrm{v} / \mathrm{v})$ was determined using a competition experiment with monofluorocyclooctyne 7. $N, N$-diethylhydroxylamine (1 equiv; $1.0 \mathrm{mM}$ final concentration) was added to a solution containing a 1:3 ratio of difluorocyclooctyne 10 (5 equiv; $5.0 \mathrm{mM}$ final concentration) and monofluorocyclooctyne 7 (15 equiv; $15 \mathrm{mM}$ final concentration) in $50 \%$ $\mathrm{H}_{2} \mathrm{O} / \mathrm{CD}_{3} \mathrm{CN}$ (v/v) at room temperature (Figure S2C). The solution was transferred to an NMR tube and the product ratio (S14:S12) was determined by ${ }^{19} \mathrm{~F}$ NMR spectroscopy using benzotrifluoride as an internal standard. The second order rate constant $\left(k_{2}\right)$ of difluorocyclooctyne $\mathbf{1 0}$ in $50 \%$ $\mathrm{H}_{2} \mathrm{O} / \mathrm{CD}_{3} \mathrm{CN}$ (v/v) was calculated by multiplying the observed product ratio with the second order rate constant $\left(k_{2}\right)$ of monofluorocyclooctyne 7 to give $16.6 \pm 1.1 \mathrm{M}^{-1} \mathrm{~s}^{-1}$. The reported error for the rate constant is the standard deviation of the mean for experiments performed in triplicate. 
A

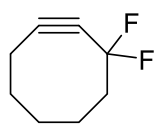

10 (9.5 mM, 5 equiv)

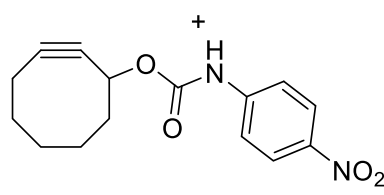

9 (38 mM, 20 equiv)

B

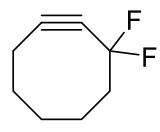

$10(10 \mathrm{mM})$

C

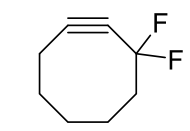

10 (5.0 mM, 5 equiv)

$+$

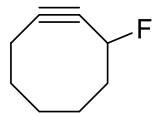

7 (15 mM, 15 equiv)

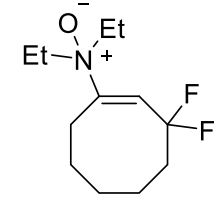

$\frac{\mathrm{NEt}_{2} \mathrm{OH}(1.9 \mathrm{mM}, 1 \text { equiv) }}{\mathrm{CD}_{3} \mathrm{CN}, \mathrm{rt}}$

$1 \mathrm{~h}$
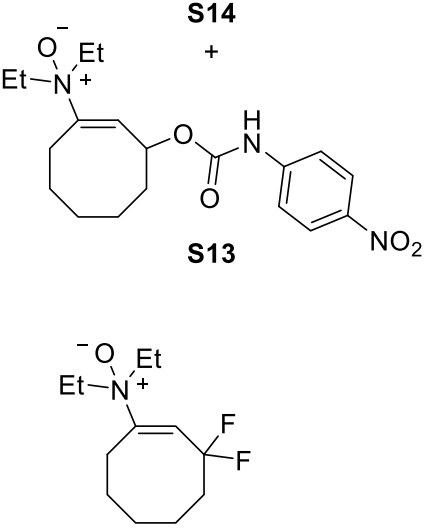

S14

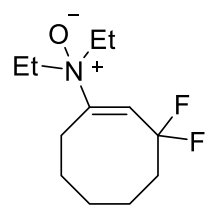

S14

$+$

$\mathrm{O}, \mathrm{Et}$

rt, $10 \mathrm{~min}$

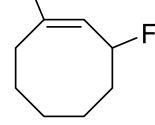

S12

Figure S2. A) Competition experiment performed between a 1:4 ratio of cyclooctyne carbamate 9 and difluorocyclooctyne $\mathbf{1 0}$ to determine the second order rate constant of the latter with $\mathrm{N}, \mathrm{N}$ diethylhydroxylamine in $\mathrm{CD}_{3} \mathrm{CN}$. B) Complete reaction conversion was observed in the presence of difluorocyclooctyne $10(10 \mathrm{mM})$ and $N, N$-diethylhydroxylamine $(10 \mathrm{mM})$ within $5 \mathrm{~min}$ in $50 \%$ $\mathrm{H}_{2} \mathrm{O} / \mathrm{CD}_{3} \mathrm{CN}$. C) Competition experiment performed between a 1:3 ratio of cyclooctyne carbamate 7 and difluorocyclooctyne $\mathbf{1 0}$ to determine the second order rate constant of the latter with $\mathrm{N}, \mathrm{N}$ diethylhydroxylamine in $50 \% \mathrm{H}_{2} \mathrm{O} / \mathrm{CD}_{3} \mathrm{CN}$. 


\section{Protein Labeling Experiments}

Synthesis of lysozyme-COT 15: Lysozyme (CAS 12650-88-3, $50 \mathrm{mg} / \mathrm{mL}$ in deionized $\mathrm{H}_{2} \mathrm{O}$ ) was diluted into phosphate-buffered saline (PBS, $\mathrm{pH} 7.4$ ) to a final concentration of $10 \mathrm{mg} / \mathrm{mL}$. A solution of cyclooctyne NHS-ester $13(65 \mu \mathrm{L}, 8.5 \mathrm{mM}$ in DMSO, 0.50 equiv) and DMSO $(10 \mu \mathrm{L})$ were added to the lysozyme solution $(250 \mu \mathrm{L}, 10 \mathrm{mg} / \mathrm{mL})$. The reaction solution was incubated for $1 \mathrm{~h}$ at room temperature. Excess cyclooctyne NHS-ester 13 was removed by spin filtration $(3 \mathrm{kDa} \mathrm{MWCO}, 5 \times$ 1:5 dilution). The concentration of lysozyme was determined by $\mathrm{A}_{280}$ measurement in denaturing buffer ( $\mathrm{pH}$ 7.0, $6 \mathrm{M}$ guanidinium, $30 \mathrm{mM}$ MOPS) on a UV-vis spectrophotometer. The solution was diluted with PBS ( $\mathrm{pH} 7.4$ ) to a final concentration of $0.15 \mathrm{mg} / \mathrm{mL}$ or $0.60 \mathrm{mg} / \mathrm{mL}$ for labeling experiments. The protein solution were snap frozen under liquid nitrogen and stored at $-20{ }^{\circ} \mathrm{C}$.

Concentration-dependent protein labeling experiments: A solution of lysozyme-COT $15(5.0 \mu \mathrm{L}$, $0.15 \mathrm{mg} / \mathrm{mL}$ ) was aliquoted each into 6 samples. Then, an aqueous solution of hydroxylamine 13 $(0.21 \mu \mathrm{L} ; 0.25,0.625,1.25,2.5$, and $5 \mathrm{mM}$ in deionized water; final concentrations of $10,25,50,100$, and $200 \mu \mathrm{M})$ was added to each of 5 aliquoted samples. Deionized water $(0.21 \mu \mathrm{L})$ was added to one sample instead of hydroxylamine as the vehicle control. Unmodified lysozyme was treated with hydroxylamine $13(0.21 \mu \mathrm{L}, 5 \mathrm{mM}$ in water; $200 \mu \mathrm{M}$ final concentration) or deionized water $(0.21$ $\mu \mathrm{L})$ in control samples requiring conditions with no lysozyme-COT 15. The reaction mixtures were incubated for $2 \mathrm{~h}$ at room temperature in the dark. The reaction mixtures were quenched with $5 \times$ SDS sample loading buffer $(1.30 \mathrm{uL})$. Each solution $(5 \mu \mathrm{L})$ was loaded onto a 15 -well 12\% SDS-PAGE gel. The gel was run at room temperature and at $175 \mathrm{~V}$ for $50 \mathrm{~min}$. In-gel fluorescence was imaged with a Typhoon FLA 9500 (GE) at $532 \mathrm{~nm}$ with a photomultiplier tube (PMT) setting of $500 \mathrm{~V}$. The experiment was carried out in triplicate.

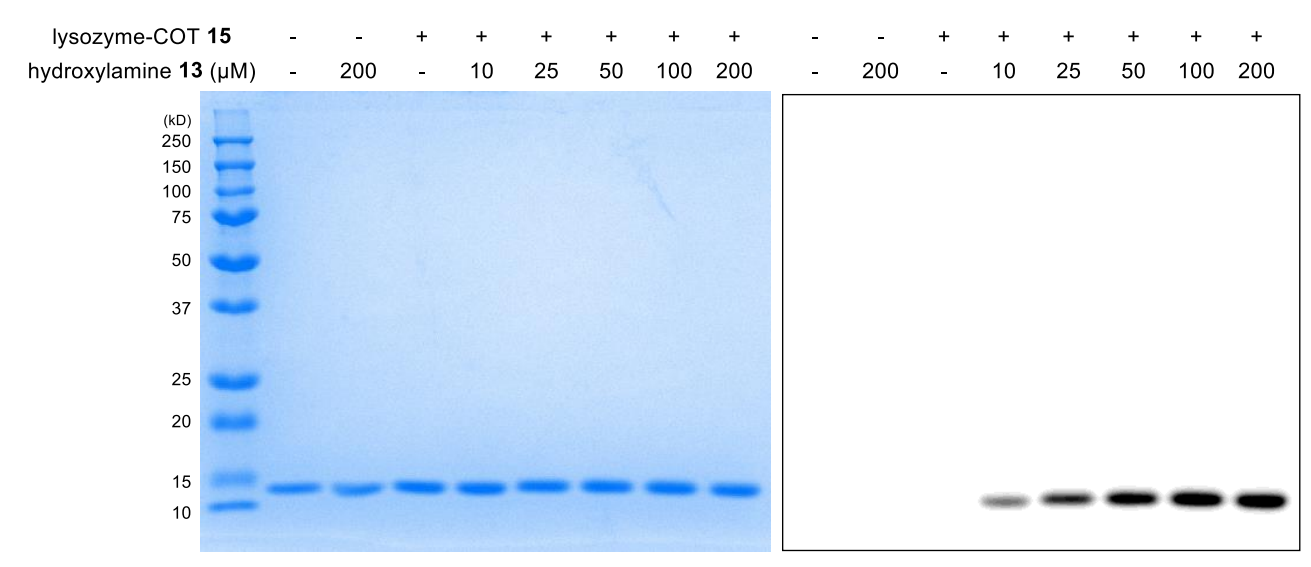

Figure S3. Full coomassie stain (left) and in-gel fluorescence (right) images for concentrationdependent protein labeling experiments. Both images are from the same gel.

Time-dependent protein labeling experiments: A solution of lysozyme-COT $15(5.0 \mu \mathrm{L}, 0.15$ $\mathrm{mg} / \mathrm{mL})$ was aliquoted each into 6 samples. Then, an aqueous solution of hydroxylamine $13(0.21 \mu \mathrm{L}$, $5 \mathrm{mM}$ in deionized water; $200 \mu \mathrm{M}$ final concentration) was added to each of 5 aliquoted samples. Deionized water $(0.21 \mu \mathrm{L})$ was added instead of hydroxylamine $\mathbf{1 3}$ to one sample for the vehicle control. Unmodified lysozyme was treated with hydroxylamine $13(0.21 \mu \mathrm{L}, 5 \mathrm{mM}$ in water; $200 \mu \mathrm{M}$ 
final concentration) or deionized water $(0.21 \mu \mathrm{L})$ in control samples requiring conditions with no lysozyme-COT 15. The reaction mixtures were incubated at room temperature in the dark and quenched by adding $N, N$-diethylhydroxylamine $\left(1.30 \mu \mathrm{L}, 100 \mathrm{mM}\right.$ in deionized $\mathrm{H}_{2} \mathrm{O} ; 20 \mathrm{mM}$ final concentration) followed by $5 \times$ SDS sample loading buffer $(1.63 \mathrm{uL})$ at each indicated time point. Samples were snap frozen under liquid nitrogen until all samples were ready to be loaded on the gel. After $2 \mathrm{~h}$, all reactions had been quenched. All samples were thawed and each solution $(5 \mu \mathrm{L})$ was loaded onto a 15 -well $12 \%$ SDS-PAGE gel. The gel was run at room temperature and at $175 \mathrm{~V}$ for 50 min. In-gel fluorescence was imaged with a Typhoon FLA 9500 (GE) at $532 \mathrm{~nm}$ with a photomultiplier tube (PMT) setting of $500 \mathrm{~V}$. The experiment was carried out in triplicate.

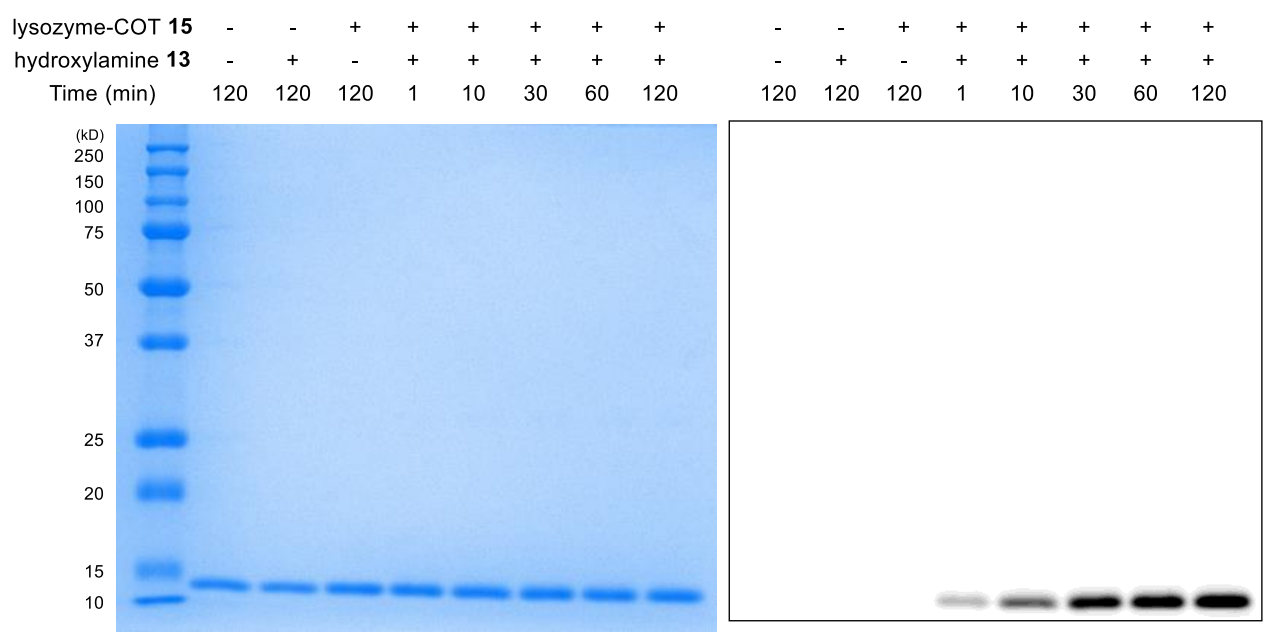

Figure S4. Full coomassie stain (left) and in-gel fluorescence (right) images for concentrationdependent protein labeling experiments. Both images are from the same gel. 


\section{Intact Mass Spectrometry Analysis}

A solution of hydroxylamine $13(0.83 \mu \mathrm{L}, 5 \mathrm{mM}$ in deionized water) was added to a solution of lysozyme-COT $15(20 \mu \mathrm{L}, 0.60 \mathrm{mg} / \mathrm{mL}$ in deionized water) to generate the reaction sample. Deionized $\mathrm{H}_{2} \mathrm{O}(0.83 \mu \mathrm{L})$ was added to lysozyme-COT $15(20 \mu \mathrm{L}, 0.60 \mathrm{mg} / \mathrm{mL}$ in deionized water $)$ to generate the vehicle control. Unmodified lysozyme $(20 \mu \mathrm{L}, 0.60 \mathrm{mg} / \mathrm{mL}$ in deionized water) was added to deionized water $(0.83 \mu \mathrm{L})$ to generate the blank background sample. Reactions were incubated at room temperature for $6 \mathrm{~h}$ in the dark. The samples were snap frozen using liquid nitrogen and stored at $-80{ }^{\circ} \mathrm{C}$ until further analysis. ESI-MS analysis was performed on an LTQ XL ion trap mass spectrometer (ThermoFisher Scientific, San Jose, CA).

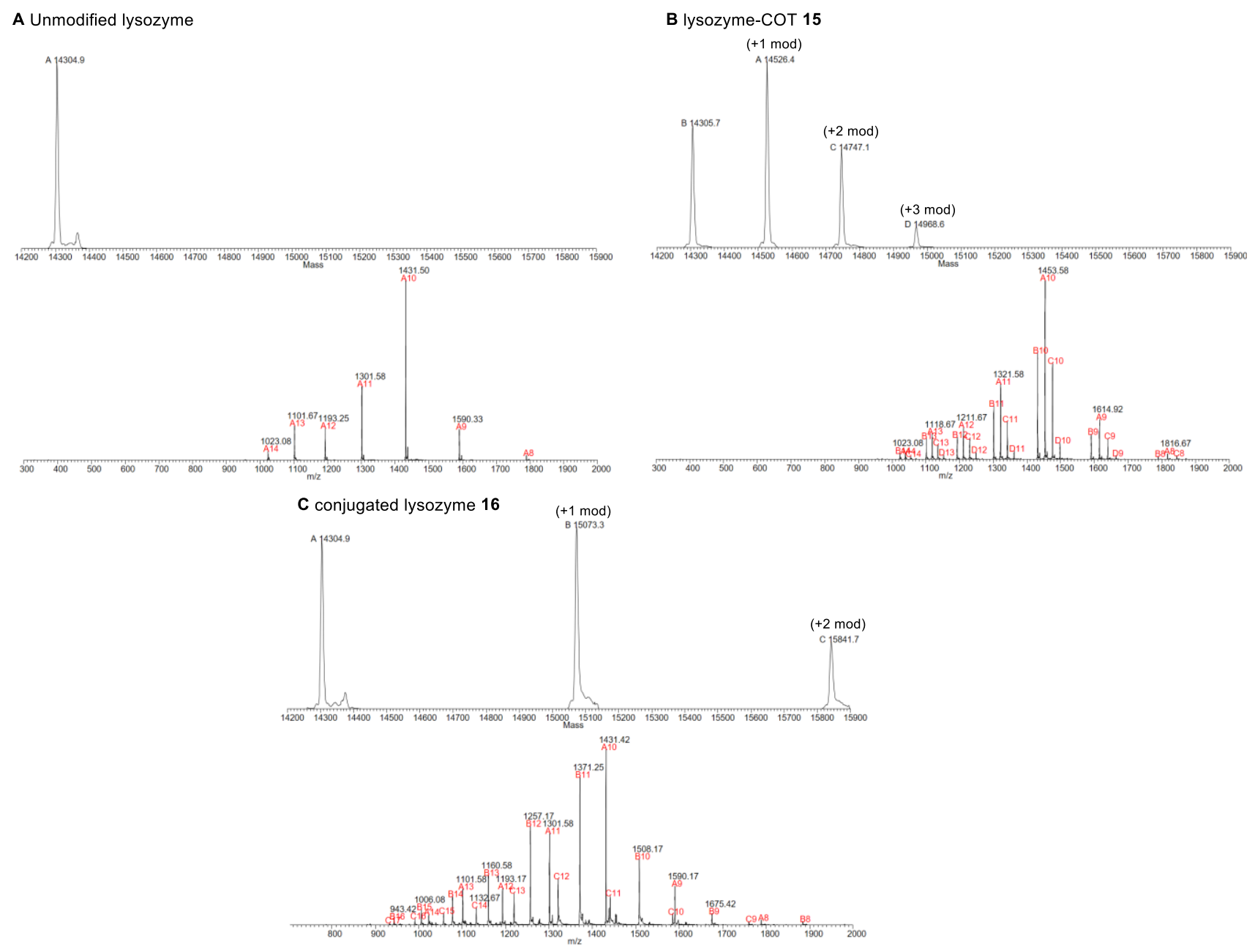

Figure S5. Mass spectrometry confirmation of the bioorthogonal reaction between hydroxylamine $\mathbf{1 3}$ and lysozyme-COT 15. ESI mass spectra of A) unmodified lysozyme, B) lysozyme-cyclooctyne conjugate, and $\mathrm{C}$ ) reaction mixture of hydroamination between hydroxylamine $\mathbf{1 3}$ and lysozymecyclooctyne conjugate 15 (single adduct: expected 15073.3 Da, observed 15073.3 Da; double adduct: expected 15840.6 Da, observed 15841.7 Da). 


\section{Protein Labeling Experiments in the Presence of Cell Lysate}

Cell Culture: HEK-293T cells were cultured in Dulbecco's Modified Eagle Media (DMEM, Corning) containing $10 \%$ fetal bovine serum (FBS, Sigma), 100 units $/ \mathrm{mL}$ penicillin, and $0.1 \mathrm{mg} / \mathrm{mL}$ streptomycin (Sigma) in a humidified chamber at $37{ }^{\circ} \mathrm{C}$ with $5 \% \mathrm{CO}_{2}$. Cells were passaged and dissociated with $0.25 \%$ trypsin, $0.1 \%$ EDTA in HBSS (Corning). Cells tested negative for mycobacteria by the MycoAlert PLUS Mycoplasma Detection Kit (Lonza) following the manufacturer's protocol.

Cell Lysate: Cell culture media was aspirated prior to lysis. Cells (10 cm dish, $\sim 80 \%$ confluency) was lysed by adding lysis buffer $\left(1.0 \mathrm{~mL}, 4{ }^{\circ} \mathrm{C} ; 150 \mathrm{mM} \mathrm{NaCl}, 50 \mathrm{mM}\right.$ Tris $(\mathrm{pH} 8.0), 1 \%$ triton X-100). After centrifugation $(13,000 \times \mathrm{g})$ at $4{ }^{\circ} \mathrm{C}$, the supernatant was transferred to a clean tube, and the protein concentration was determined by BCA (bicinchoninic acid) protein assay (Pierce ${ }^{\mathrm{TM}} \mathrm{BCA}$ Protein Assay Kit). Aliquots of the cell lysate $(7.1 \mathrm{mg} / \mathrm{mL})$ were snap frozen under liquid nitrogen and stored at $-20{ }^{\circ} \mathrm{C}$.

Protein Labeling Experiments: Lysozyme-COT $15(0.75 \mu \mathrm{g}, 5.0 \mu \mathrm{L}, 0.15 \mathrm{mg} / \mathrm{mL}$ in deionized water) was aliquoted to form 4 samples. Cell lysate $(20 \mu \mathrm{g}, 2.83 \mu \mathrm{L}, 7.1 \mathrm{mg} / \mathrm{mL})$ was added to 2 samples, and deionized water $(2.83 \mu \mathrm{L})$ was added to the remaining 2 samples. A fifth control sample lacking lysozyme-COT 15 was prepared by adding cell lysate $(20 \mu \mathrm{g}, 2.83 \mu \mathrm{L}, 7.1 \mathrm{mg} / \mathrm{mL})$ to deionized water $(5.0 \mu \mathrm{L})$. Then, either hydroxylamine $13(0.33 \mu \mathrm{L}, 5 \mathrm{mM}$ in deionized water) or deionized water $(0.33 \mu \mathrm{L})$ were added to the samples according to the conditions laid out in Figure 5B. The reaction mixtures were incubated for $2 \mathrm{~h}$ at room temperature in the dark. The reaction mixtures were quenched with $5 \times$ SDS sample loading buffer $(1.30 \mathrm{uL})$. Each solution $(5 \mu \mathrm{L})$ was loaded onto a 15 well 12\% SDS-PAGE gel. The gel was run at room temperature and at $175 \mathrm{~V}$ for 50 min. In-gel fluorescence was imaged with a Typhoon FLA 9500 (GE) at $532 \mathrm{~nm}$ with a photomultiplier tube (PMT) setting of $500 \mathrm{~V}$. The experiment was carried out in triplicate. 


\section{Stability Studies}

All reactions were monitored by HPLC at $0,1,2,4,8$, and $24 \mathrm{~h}$ time points.

HPLC analysis: Reactions with enamine $N$-oxide 17 were analyzed by HPLC (Pursuit $200 \AA \mathrm{C}_{18}, 4.6$ $\times 150 \mathrm{~mm}, 10 \mu \mathrm{m}$ particles, $1 \mathrm{~mL} / \mathrm{min}$ flow rate, eluent: isocratic $0 \% \mathrm{MeCN} / \mathrm{H}_{2} \mathrm{O}+0.1 \% \mathrm{TFA}(1$ min), gradient $0 \rightarrow 100 \% \mathrm{MeCN} / \mathrm{H}_{2} \mathrm{O}+0.1 \%$ TFA (14 min), isocratic $100 \% \mathrm{MeCN} / \mathrm{H}_{2} \mathrm{O}+0.1 \%$ TFA $(1 \mathrm{~min})$ ) and quantified using its absorbance at $280 \mathrm{~nm}$. Reactions with hydroxylamine 13 were analyzed by HPLC (Pursuit $200 \AA \mathrm{C}_{18}, 4.6 \times 150 \mathrm{~mm}, 10 \mu \mathrm{m}$ particles, $1 \mathrm{~mL} / \mathrm{min}$ flow rate, eluent: isocratic $0 \% \mathrm{MeCN} / \mathrm{H}_{2} \mathrm{O}+0.1 \%$ TFA $(1 \mathrm{~min})$, gradient $0 \rightarrow 20 \% \mathrm{MeCN} / \mathrm{H}_{2} \mathrm{O}+0.1 \% \mathrm{TFA}(1 \mathrm{~min})$, gradient $20 \rightarrow 50 \% \mathrm{MeCN} / \mathrm{H}_{2} \mathrm{O}+0.1 \%$ TFA (16 min), gradient $50 \rightarrow 100 \% \mathrm{MeCN} / \mathrm{H}_{2} \mathrm{O}+0.1 \%$ TFA (2 min), isocratic $100 \% \mathrm{MeCN} / \mathrm{H}_{2} \mathrm{O}+0.1 \%$ TFA $\left.(1 \mathrm{~min})\right)$ and quantified using its absorbance at 254 $\mathrm{nm}$.

Stability in PBS: Enamine $N$-oxide 17 ( $4 \mu \mathrm{L}, 15 \mathrm{mM}$ in 25\% v/v methanol/PBS, $\mathrm{pH} 7.4$ ) was added to PBS $(116 \mu \mathrm{L}, \mathrm{pH} 7.4 ; 500 \mu \mathrm{M}$ final concentration). Separately, hydroxylamine 13 (3 $\mu \mathrm{L}, 20 \mathrm{mM}$ in deionized $\left.\mathrm{H}_{2} \mathrm{O}\right)$ was added to $\mathrm{PBS}(117 \mu \mathrm{L}, \mathrm{pH} 7.4 ; 500 \mu \mathrm{M}$ final concentration). Each solution was monitored by HPLC at each time point .

Stability in the presence of glutathione, sodium ascorbate, and cell lysate: The reactions were conducted as described above in the Stability in PBS section except the PBS solution was first supplemented with glutathione (5 $\mathrm{mM}$ final concentration), sodium ascorbate (5 $\mathrm{mM}$ final concentration), or HEK293T cell lysate (1 mg/mL final concentration) and $\mathrm{pH}$ adjusted to 7.4 before adding enamine $N$-oxide $17(500 \mu \mathrm{M}$ final concentration) or hydroxylamine $13(500 \mu \mathrm{M}$ final concentration).

Microsomal assay I: A solution of human liver microsomes $(8 \mu \mathrm{L}, 20 \mathrm{mg} / \mathrm{mL}$ in phosphate buffer, $\mathrm{pH}$ 7.4, Corning; $200 \mu \mathrm{g} / \mathrm{mL}$ final concentration) and a solution of NADPH (13.4 $\mu \mathrm{L}, 60 \mathrm{mM}$ in $10 \mathrm{mM}$ $\mathrm{NaOH}$ solution) were sequentially added to PBS $(751.9 \mu \mathrm{L}, \mathrm{pH} 7.4)$ in a $2.0 \mathrm{~mL}$ microcentrifuge tube. The solution was incubated for $1 \mathrm{~h}$ at room temperature to provide solution A. A solution of enamine $N$-oxide $17(26.7 \mu \mathrm{L}, 15 \mathrm{mM}$ in 25\% methanol/PBS, pH 7.4; $500 \mu \mathrm{M}$ final concentration) was added to solution A. The cap of the microcentrifuge tube was pierced with a $16 \mathrm{G}$ needle to maintain an aerobic system. The reaction was incubated at room temperature in the dark. At each time point, $100 \mu \mathrm{L}$ of the sample was transferred to a clean $2.0 \mathrm{~mL}$ microcentrifuge tube, and the reaction was quenched with acetonitrile $(100 \mu \mathrm{L})$. The mixture was centrifuged $(13,000 \times \mathrm{g})$ at $4{ }^{\circ} \mathrm{C}$ for $5 \mathrm{~min}$, then the supernatant was transferred to a clean HPLC vial for analysis.

Microsomal assay II: A solution of human liver microsomes $(8 \mu \mathrm{L}, 20 \mathrm{mg} / \mathrm{mL}$ in phosphate buffer, $\mathrm{pH} 7.4$, Corning; $200 \mu \mathrm{g} / \mathrm{mL}$ final concentration) and a solution of NADPH (13.4 $\mu \mathrm{L}, 60 \mathrm{mM}$ in 10 $\mathrm{mM} \mathrm{NaOH}$ solution; $1 \mathrm{mM}$ final concentration) were sequentially added to PBS (358.6 $\mu \mathrm{L}, \mathrm{pH} 7.4$ ) and incubated at room temperature for $1 \mathrm{~h}$ to provide solution B. A solution of sodium ascorbate (400 $\mu \mathrm{L}, 10 \mathrm{mM}$ in PBS, pH 7.4) and a solution of hydroxylamine $\mathbf{1 3}\left(20 \mu \mathrm{L}, 20 \mathrm{mM}\right.$ in deionized $\left.\mathrm{H}_{2} \mathrm{O}\right)$ were added to solution B. The cap of the microcentrifuge tube was pierced with a $16 \mathrm{G}$ needle to maintain an aerobic system. The reaction was incubated at room temperature in the dark. At each time point, $100 \mu \mathrm{L}$ of the sample was transferred to a clean $2.0 \mathrm{~mL}$ microcentrifuge tube, and the reaction was quenched with acetonitrile $(100 \mu \mathrm{L})$. The mixture was centrifuged $(13,000 \times \mathrm{g})$ at $4{ }^{\circ} \mathrm{C}$ for 5 min and the supernatant was transferred to a clean HPLC vial for analysis. 


\section{Cross Reactivity Studies}

Cyclooctynes with Me-tetrazine: A solution of cyclooctyne 5, 9, or $\mathbf{1 0}\left(125 \mu \mathrm{L}, 20 \mathrm{mM}\right.$ in $\mathrm{CD}_{3} \mathrm{CN}, 1$ equiv; $5 \mathrm{mM}$ final concentration) was each added to a separate NMR tube. A solution containing the internal standard 1,3,5-trimethoxybenzene (TMB, $50 \mu \mathrm{L}, 50 \mathrm{mM}$ in $\mathrm{CD}_{3} \mathrm{CN}$; $5 \mathrm{mM}$ final concentration) was then added via syringe to each of the samples. $\mathrm{CD}_{3} \mathrm{CN}(275 \mu \mathrm{L})$ was added to each tube to bring the volume of each solution to $450 \mu \mathrm{L}$. The tubes were inverted three times to mix the solutions, and reference spectra were obtained by ${ }^{1} \mathrm{H}$ NMR spectroscopy. A solution of tert-butyl(4-(6-methyl-1,2,4,5-tetrazin-3-yl)benzyl)carbamate (S18, $50 \mu \mathrm{L}, 50 \mathrm{mM}$ in $\mathrm{CD}_{3} \mathrm{CN}, 2.00$ equiv; 10 $\mathrm{mM}$ final concentration) was added to bring the final volume to $500 \mu \mathrm{L}$. The tubes were immediately inverted three times to mix the reaction solutions then incubated for $1 \mathrm{~h}$ at room temperature. The reaction mixtures were analyzed by ${ }^{1} \mathrm{H}$ NMR spectroscopy. No change in the spectra was observed.
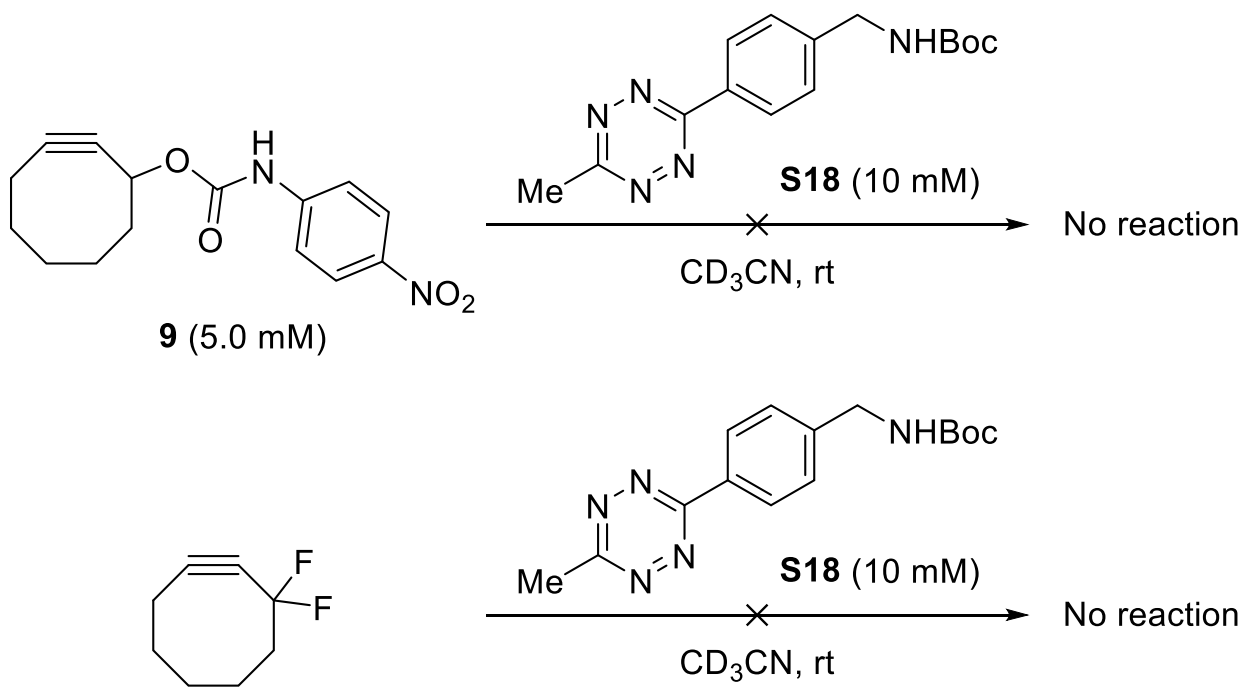

$10(5.0 \mathrm{mM})$
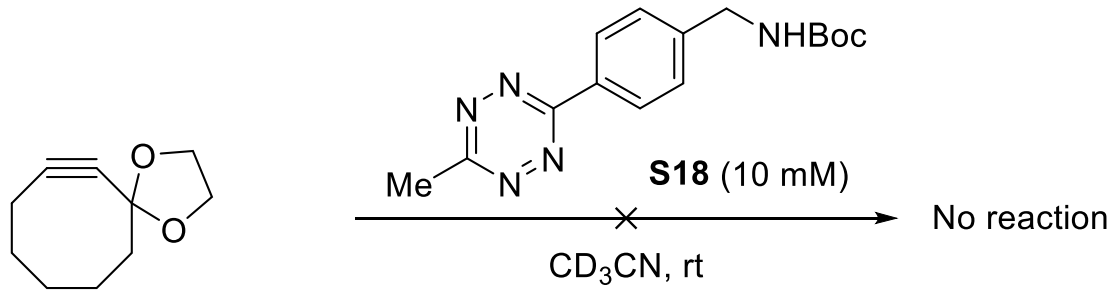

$5(5.0 \mathrm{mM})$

Figure S6. Cross-reactivity between cyclooctynes 5, 9, and $\mathbf{1 0}$ and Me-tetrazine $\mathbf{S 1 8}$ was evaluated in $\mathrm{CD}_{3} \mathrm{CN}$ at room temperature.

Hydroxylamines with various components: A solution of (E)-cyclooct-4-en-1-yl (3aminopropyl)carbamate (S19, $16 \mathrm{mM}$ in $6.2 \% \mathrm{D}_{2} \mathrm{O}$ in $\mathrm{CD}_{3} \mathrm{CN} ; 5 \mathrm{mM}$ final concentration), 4methylbenzaldehyde (S20, $100 \mathrm{mM}$ in $\mathrm{CD}_{3} \mathrm{CN}$; $5 \mathrm{mM}$ final concentration), (2-methylcycloprop-2-en1-yl)methyl isopropylcarbamate (S21, $50 \mathrm{mM}$ in $\mathrm{CD}_{3} \mathrm{CN} ; 5 \mathrm{mM}$ final concentration), or hex-5-ynoic acid (S22, $50 \mathrm{mM}$ in $\mathrm{CD}_{3} \mathrm{CN} ; 5 \mathrm{mM}$ final concentration) was each added to a separate NMR tube. A solution containing the internal standard 1,3,5-trimethoxybenzene (TMB, $50 \mu \mathrm{L}, 50 \mathrm{mM}$ in $\mathrm{CD}_{3} \mathrm{CN}_{\text {; }}$ $5 \mathrm{mM}$ final concentration) was then added via syringe to each of the samples. $\mathrm{CD}_{3} \mathrm{CN}$ was added to each tube to bring the volume of each solution to $475 \mu \mathrm{L}$. The tubes were inverted three times to mix 
the solutions, and reference spectra were obtained by ${ }^{1} \mathrm{H}$ NMR spectroscopy. For each reaction, a solution of $N, N$-diethylhydroxylamine $\left(25 \mu \mathrm{L}, 100 \mathrm{mM}\right.$ in $\mathrm{CD}_{3} \mathrm{CN}, 2.00$ equiv; $10 \mathrm{mM}$ final concentration) was added to bring the final volume to $500 \mu \mathrm{L}$. The tubes were immediately inverted three times to mix the reaction solutions then incubated for $1 \mathrm{~h}$ at room temperature. The reaction mixtures were analyzed by ${ }^{1} \mathrm{H}$ NMR spectroscopy. No change in the spectra was observed.
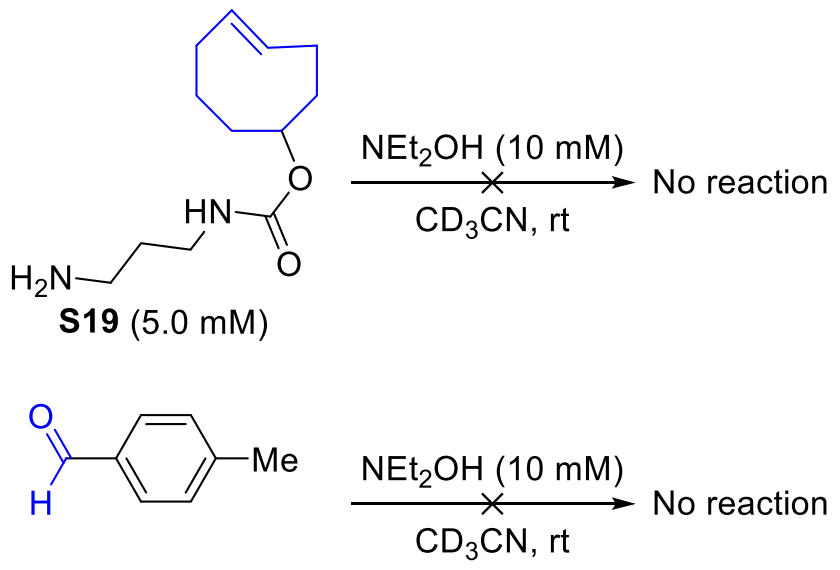

$\mathbf{S 2 0}(5.0 \mathrm{mM})$<smiles>CC(C)NC(=O)OCC1CC1</smiles>

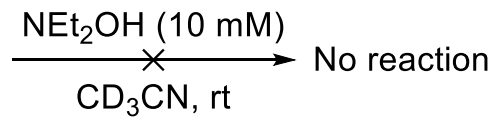

$\mathbf{S 2 1}(5.0 \mathrm{mM})$

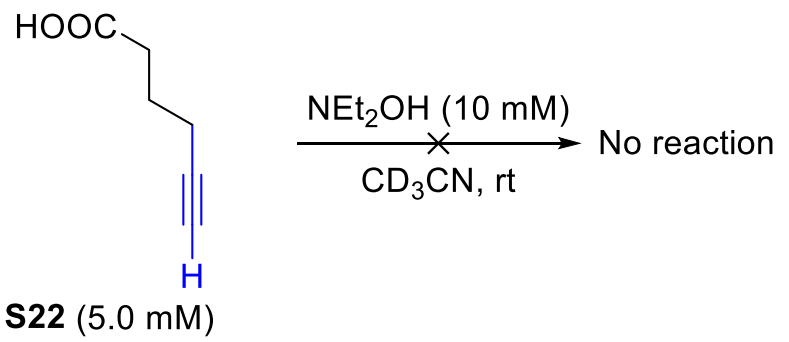

Figure S7. Cross-reactivity between $N, N$-diethylhydroxylamine and bioorthogonal components S19S22 was evaluated in $\mathrm{CD}_{3} \mathrm{CN}$ at room temperature.

Cu-catalyzed azide-alkyne cycloaddition (CuAAC): A $2.0 \mathrm{mM}$ microcentrifuge tube was sequentially charged with solutions of 3',6'-bis(dimethylamino)-3-oxo- $N$-(prop-2-yn-1-yl)-3Hspiro[isobenzofuran-1,9'-xanthene]-5-carboxamide (S23, Click Chemistry Tools 1255-5, $8 \mu \mathrm{L}, 5 \mathrm{mM}$ in DMSO, 1 equiv), 3,3 ',3 ' ' $-\left(4,4^{\prime}, 4^{\prime}{ }^{\prime}\right.$-(nitrilotris(methylene))tris(1H-1,2,3-triazole-4,1diyl))tris(propan-1-ol) (THPTA, $0.6 \mu \mathrm{L}, 100 \mathrm{mM}$ in DMSO, 1.50 equiv), $\mathrm{CuSO}_{4} \cdot \mathrm{H}_{2} \mathrm{O}(0.4 \mu \mathrm{L}, 50$ $\mathrm{mM}$ in PBS, $\mathrm{pH} 7.4,0.500$ equiv), sodium ascorbate $(10 \mu \mathrm{L}, 100 \mathrm{mM}$ in $\mathrm{PBS}, \mathrm{pH} 7.4,25.0$ equiv), and PBS $(180.2 \mu \mathrm{L}, \mathrm{pH} 7.4)$ at room temperature. A solution of $N, N$-diethylhydroxylamine $(0.8 \mu \mathrm{L}$, $100 \mathrm{mM}$ in PBS, pH 7.4, 2.00 equiv) was added to this mixture to bring the total volume to $200 \mu \mathrm{L}$. The solution was immediately transferred to a clean HPLC vial to monitor the reaction progress and incubated at room temperature in the dark. The reaction was analyzed by HPLC (Pursuit $200 \AA \mathrm{C}_{18}$, 
$4.6 \times 150 \mathrm{~mm}, 10 \mu \mathrm{m}$ particles, $1 \mathrm{~mL} / \mathrm{min}$ flow rate, eluent: isocratic $0 \% \mathrm{MeCN} / \mathrm{H}_{2} \mathrm{O}+0.1 \% \mathrm{TFA}(1$ min), gradient $0 \rightarrow 100 \% \mathrm{MeCN} / \mathrm{H}_{2} \mathrm{O}+0.1 \%$ TFA (14 min), isocratic $100 \% \mathrm{MeCN} / \mathrm{H}_{2} \mathrm{O}+0.1 \%$ TFA $(1 \mathrm{~min}))$ at each time point $(0,1,2$, and $4 \mathrm{~h})$ and quantified using its absorbance at $280 \mathrm{~nm}$. No reaction was observed.

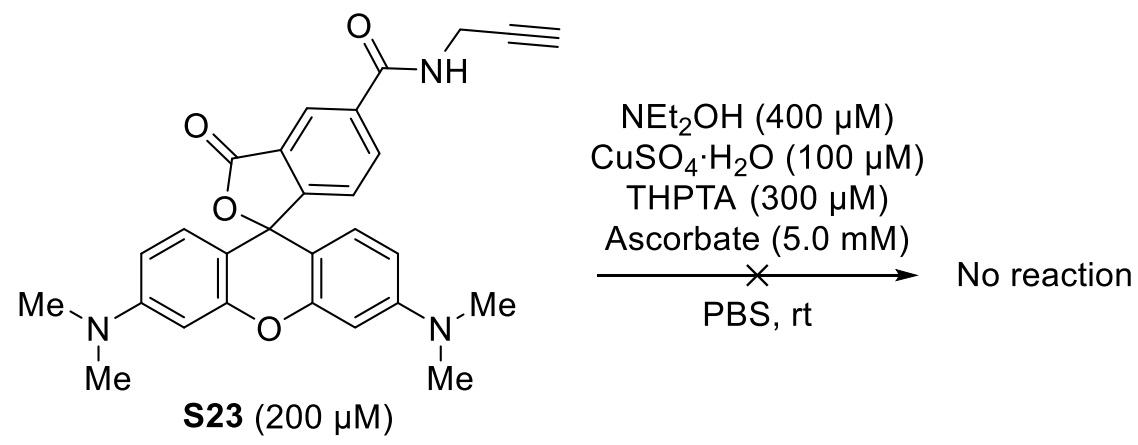

Figure S8. Cross-reactivity between $N, N$-diethylhydroxylamine and alkyne $\mathbf{S 2 3}$ was evaluated in PBS at room temperature. 


\section{Live Cell Dual Labeling Experiment}

pHTC-Igк chain leader seq-HaloTag-PDGFR-sfGFP:

ATGGAGACAGACACACTCCTGCTATGGGTACTGCTGCTCTGGGTTCCAGGTTCCACTGGT GACTATCCATATGATGTTCCAGATTATGCTGGATCCGAAATCGGTACTGGCTTTCCATTC GACCCCCATTATGTGGAAGTCCTGGGCGAGCGCATGCACTACGTCGATGTTGGTCCGCG CGATGGCACCCCTGTGCTGTTCCTGCACGGTAACCCGACCTCCTCCTACGTGTGGCGCAA CATCATCCCGCATGTTGCACCGACCCATCGCTGCATTGCTCCAGACCTGATCGGTATGGG CAAATCCGACAAACCAGACCTGGGTTATTTCTTCGACGACCACGTCCGCTTCATGGATGC CTTCATCGAAGCCCTGGGTCTGGAAGAGGTCGTCCTGGTCATTCACGACTGGGGCTCCGC TCTGGGTTTCCACTGGGCCAAGCGCAATCCAGAGCGCGTCAAAGGTATTGCATTTATGG AGTTCATCCGCCCTATCCCGACCTGGGACGAATGGCCAGAATTTGCCCGCGAGACCTTCC AGGCCTTCCGCACCACCGACGTCGGCCGCAAGCTGATCATCGATCAGAACGTTTTTATCG AGGGTACGCTGCCGATGGGTGTCGTCCGCCCGCTGACTGAAGTCGAGATGGACCATTAC CGCGAGCCGTTCCTGAATCCTGTTGACCGCGAGCCACTGTGGCGCTTCCCAAACGAGCTG CCAATCGCCGGTGAGCCAGCGAACATCGTCGCGCTGGTCGAAGAATACATGGACTGGCT GCACCAGTCCCCTGTCCCGAAGCTGCTGTTCTGGGGCACCCCAGGCGTTCTGATCCCACC GGCCGAAGCCGCTCGCCTGGCCAAAAGCCTGCCTAACTGCAAGGCTGTGGACATCGGCC CGGGTCTGAATCTGCTGCAAGAAGACAACCCGGACCTGATCGGCAGCGAGATCGCGCGC TGGCTGTCTACTCTGGAGATTTCCGGTGAAAACCTGTACTTCCAATCCGCTGTGGGCCAG GACACGCAGGAGGTCATCGTGGTGCCACACTCCTTGCCCTTTAAGGTGGTGGTGATCTCA GCCATCCTGGCCCTGGTGGTGCTCACCATCATCTCCCTTATCATCCTCATCATGCTTTGGC AGAAGAAGCCACGTGGTGGTTCTGGTATGGTTAGCAAAGGTGAAGAACTGTTTACCGGC GTTGTGCCGATTCTGGTGGAACTGGATGGTGATGTGAATGGCCATAAATTTAGCGTTCGT GGCGAAGGCGAAGGTGATGCGACCAACGGTAAACTGACCCTGAAATTTATTTGCACCAC CGGTAAACTGCCGGTTCCGTGGCCGACCCTGGTGACCACCCTGACCTATGGCGTTCAGTG CTTTAGCCGCTATCCGGATCATATGAAACGCCATGATTTCTTTAAAAGCGCGATGCCGGA AGGCTATGTGCAGGAACGTACCATTAGCTTCAAAGATGATGGCACCTATAAAACCCGTG CGGAAGTTAAATTTGAAGGCGATACCCTGGTGAACCGCATTGAACTGAAAGGTATTGAT TTTAAAGAAGATGGCAACATTCTGGGTCATAAACTGGAATATAATTTCAACAGCCATGC GGTGTATATTACCGCCGATAAACAGAAAAATGGCATCAAAGCGAACTTTAAAATCCGTC ACAACGTGGAAGATGGTAGCGTGCAGCTGGCGGATCATTATCAGCAGAATACCCCGATT GGTGATGGCCCGGTGCTGCTGCCGGATAATCATTATCTGAGCACCCAGAGCGTTCTGAG CAAAGATCCGAATGAAAAACGTGATCATATGGTGCTGCTGGAATTTGTTACCGCCGCGG GCATTACCCACGGTATGGATGAACTGTATAAAGGCAGC

pHTC-Igк chain leader seq-SnapTag-PDGFR-TagBFP2:

ATGGAGACAGACACACTCCTGCTATGGGTACTGCTGCTCTGGGTTCCAGGTTCCACTGGT GACTATCCATATGATGTTCCAGATTATGCTATGGACAAAGACTGCGAAATGAAGCGCAC CACCCTGGATAGCCCTCTGGGCAAGCTGGAACTGTCTGGGTGCGAACAGGGCCTGCACC GTATCATCTTCCTGGGCAAAGGAACATCTGCCGCCGACGCCGTGGAAGTGCCTGCCCCA GCCGCCGTGCTGGGCGGACCAGAGCCACTGATGCAGGCCACCGCCTGGCTCAACGCCTA CTTTCACCAGCCTGAGGCCATCGAGGAGTTCCCTGTGCCAGCCCTGCACCACCCAGTGTT CCAGCAGGAGAGCTTTACCCGCCAGGTGCTGTGGAAACTGCTGAAAGTGGTGAAGTTCG GAGAGGTCATCAGCTACAGCCACCTGGCCGCCCTGGCCGGCAATCCCGCCGCCACCGCC GCCGTGAAAACCGCCCTGAGCGGAAATCCCGTGCCCATTCTGATCCCCTGCCACCGGGT GGTGCAGGGCGACCTGGACGTGGGGGGCTACGAGGGCGGGCTCGCCGTGAAAGAGTGG 
CTGCTGGCCCACGAGGGCCACAGACTGGGCAAGCCTGGGCTGGGTGAAAACCTGTACTT CCAATCCGCTGTGGGCCAGGACACGCAGGAGGTCATCGTGGTGCCACACTCCTTGCCCTT TAAGGTGGTGGTGATCTCAGCCATCCTGGCCCTGGTGGTGCTCACCATCATCTCCCTTAT CATCCTCATCATGCTTTGGCAGAAGAAGCCACGTGGTGGTTCTGGTATGAGCGAGCTGAT TAAGGAGAACATGCACATGAAGCTGTACATGGAGGGCACCGTGGACAACCATCACTTCA AGTGCACATCCGAGGGCGAAGGCAAGCCCTACGAGGGCACCCAGACCATGAGAATCAA GGTGGTCGAGGGCGGCCCTCTCCCCTTCGCCTTCGACATCCTGGCTACTAGCTTCCTCTA CGGCAGCAAGACCTTCATCAACCACACCCAGGGCATCCCCGACTTCTTCAAGCAGTCCTT CCCTGAGGGCTTCACATGGGAGAGAGTCACCACATACGAAGACGGGGGCGTGCTGACCG CTACCCAGGACACCAGCCTCCAGGACGGCTGCCTCATCTACAACGTCAAGATCAGAGGG GTGAACTTCACATCCAACGGCCCTGTGATGCAGAAGAAAACACTCGGCTGGGAGGCCTT CACCGAGACGCTGTACCCCGCTGACGGCGGCCTGGAAGGCAGAAACGACATGGCCCTGA AGCTCGTGGGCGGGAGCCATCTGATCGCAAACGCCAAGACCACATATAGATCCAAGAAA CCCGCTAAGAACCTCAAGATGCCTGGCGTCTACTATGTGGACTACAGACTGGAAAGAAT CAAGGAGGCCAACAACGAGACCTACGTCGAGCAGCACGAGGTGGCAGTGGCCAGATAC TGCGACCTCCCTAGCAAACTGGGGCACAAGCTTAAT

Cell culture: HeLa cells were cultured in RPMI 1640 (Corning) containing 10\% fetal bovine serum (FBS, Sigma), 100 units $/ \mathrm{mL}$ penicillin, $0.1 \mathrm{mg} / \mathrm{mL}$ streptomycin (Sigma) in a humidified chamber at $37{ }^{\circ} \mathrm{C}$ with $5 \% \mathrm{CO}_{2}$. Cells were passaged and dissociated with $0.25 \%$ trypsin, $0.1 \%$ EDTA in HBSS (Corning). Cells tested negative for mycobacteria by the MycoAlert PLUS Mycoplasma Detection Kit (Lonza) following the manufacturer's protocol.

Preparation for 12-Well Plate: Coverslips (15 mm circle, thickness $0.13-0.17 \mathrm{~mm}$ ) were acid-washed according to the Cold Spring Harbor protocol. ${ }^{6}$ A coverslip was added to each well in a 12-well plate followed by an aqueous solution of poly-D-lysine (Cultrex, $1 \mathrm{~mL}$ ). The plate was gently shaken to distribute the solution evenly, incubated at room temperature for $1 \mathrm{~h}$, washed using $1 \mathrm{~mL}$ of autoclaved deionized water and PBS (pH 7.4), and dried.

Live cell dual labeling experiment: HeLa cells were seeded at a density of 250,000 cells per well in 1 $\mathrm{mL}$ of RPMI with $10 \%$ FBS (Sigma), 100 units $/ \mathrm{mL}$ penicillin, and $0.1 \mathrm{mg} / \mathrm{mL}$ streptomycin (Sigma). Cells were then grown in a humidified chamber at $37{ }^{\circ} \mathrm{C}$ with $5 \% \mathrm{CO}_{2}$. Upon reaching $\sim 80 \%$ confluency, the cells in each well were transfected with Mirus Bio TransIT-LT1 transfection reagent $(3 \mu \mathrm{L})$ and plasmids $(0.5 \mu \mathrm{g}$ of pHTC-Igא chain leader seq-HaloTag-PDGFR-sfGFP and $0.5 \mu \mathrm{g}$ of pHTC-Igк chain leader seq-SnapTag-PDGFR-TagBFP2) in OptiMEM I reduced serum medium (100 $\mu \mathrm{L})$. After $36 \mathrm{~h}$, cells were washed with PBS supplemented with $\mathrm{Mg}^{2+}$ and $\mathrm{Ca}^{2+}(3 \times 1 \mathrm{~mL})$. Serumfree RPMI $(400 \mu \mathrm{L})$ was added to each well containing compounds $(10 \mu \mathrm{M})$ or vehicle (DMSO) as follows:

Well 1 and 2: COT-chloroalkane $18(2.00 \mu \mathrm{L}, 2 \mathrm{mM}$ stock solution in DMSO; final concentration 10 $\mu \mathrm{M})+$ TCO-benzylguanine $19(2.00 \mu \mathrm{L}, 2 \mathrm{mM}$ stock solution in DMSO; final concentration $10 \mu \mathrm{M})$ in serum-free RPMI $(400 \mu \mathrm{L})$

Well 3: COT-chloroalkane $18(2.00 \mu \mathrm{L}, 2 \mathrm{mM}$ stock solution in DMSO; final concentration $10 \mu \mathrm{M})+$ DMSO $(2.00 \mu \mathrm{L})$ in serum-free RPMI $(400 \mu \mathrm{L})$

Well 4: TCO-benzylguanine $19(2.00 \mu \mathrm{L}, 2 \mathrm{mM}$ stock solution in DMSO; final concentration $10 \mu \mathrm{M})$ + DMSO $(2.00 \mu \mathrm{L})$ in serum-free RPMI $(400 \mu \mathrm{L})$

Well 5: DMSO $(4.00 \mu \mathrm{L})$ in serum-free RMPI $(400 \mu \mathrm{L})$ 
Cells were incubated at $37{ }^{\circ} \mathrm{C}$ for $30 \mathrm{~min}$. After incubation, cells were washed with PBS supplemented with $\mathrm{Mg}^{2+}$ and $\mathrm{Ca}^{2+}(3 \times 1 \mathrm{~mL})$. Then, serum-free RPMI $(400 \mu \mathrm{L})$ was added to each well containing fluorophore ( 1 or $50 \mu \mathrm{M}$ ) or vehicle (autoclaved deionized water) as follows:

Well 1 and 5: TAMRA-hydroxylamine $13\left(2.00 \mu \mathrm{L}, 10 \mathrm{mM}\right.$ stock solution in $\mathrm{H}_{2} \mathrm{O}$; final concentration $50 \mu \mathrm{M}$ ) + Cy5-tetrazine 20 (Click Chemistry Tools 1019-1, $0.40 \mu \mathrm{L}, 1 \mathrm{mM}$ stock solution in $\mathrm{H}_{2} \mathrm{O}$; final concentration $\left.1 \mu \mathrm{M}\right)$ in serum-free RPMI $(400 \mu \mathrm{L})$

Well 2: autoclaved deionized water $(2.40 \mu \mathrm{L})$ in serum-free RPMI $(400 \mu \mathrm{L})$

Well 3: Cy5-tetrazine $20\left(0.40 \mu \mathrm{L}, 1 \mathrm{mM}\right.$ stock solution in $\mathrm{H}_{2} \mathrm{O}$; final concentration $\left.1 \mu \mathrm{M}\right)$ in serumfree RPMI $(400 \mu \mathrm{L})$

Well 4: TAMRA-hydroxylamine $13\left(2.00 \mu \mathrm{L}, 10 \mathrm{mM}\right.$ stock solution in $\mathrm{H}_{2} \mathrm{O}$; final concentration 50 $\mu \mathrm{M})$ in serum-free RPMI $(400 \mu \mathrm{L})$

Cells were incubated at $37{ }^{\circ} \mathrm{C}$ for $1 \mathrm{~h}$. After incubation, cells were washed with PBS supplemented with $\mathrm{Mg}^{2+}$ and $\mathrm{Ca}^{2+}(3 \times 1 \mathrm{~mL})$. Paraformaldehyde solution $\left(4 \% \mathrm{w} / \mathrm{v}\right.$ in $\left.\mathrm{H}_{2} \mathrm{O}, 1 \mathrm{~mL}\right)$ was then added to fix the cells, and the solution was incubated at room temperature for $20 \mathrm{~min}$. After gently washing the fixed cells with PBS supplemented with $\mathrm{Mg}^{2+}$ and $\mathrm{Ca}^{2+}(3 \times 1 \mathrm{~mL})$, each coverslip was lifted, washed by dipping twice into deionized water in a $200 \mathrm{~mL}$ beaker, and mounted on a microscopy slide using aqueous mounting media (20 mM Tris $\mathrm{pH} 8.0,0.5 \% \mathrm{~N}$-propyl gallate, $90 \%$ glycerol).

Fluorescence microscopy: Slides were imaged at the Confocal and Light Microscopy Core at DanaFarber Cancer Institute using a Nikon Ti Eclipse. Images were acquired with a $40 \times$ objective at 0.16 micron/pixel using a Hamamatsu camera. BFP was imaged with a 405/20 filter (excitation) and a 460/50 filter (emission) and false-colored blue; GFP was imaged with a 482/35 filter (excitation) and a 536/75 filter (emission) and false-colored green; TAMRA was imaged with a 560/40 filter (excitation) and a 630/75 filter (emission) and false-colored red; and Cy5 was imaged with a 620/60 filter (excitation) and a 700/75 filter (emission) and false-colored cyan. Images were processed with Fiji ImageJ software. 


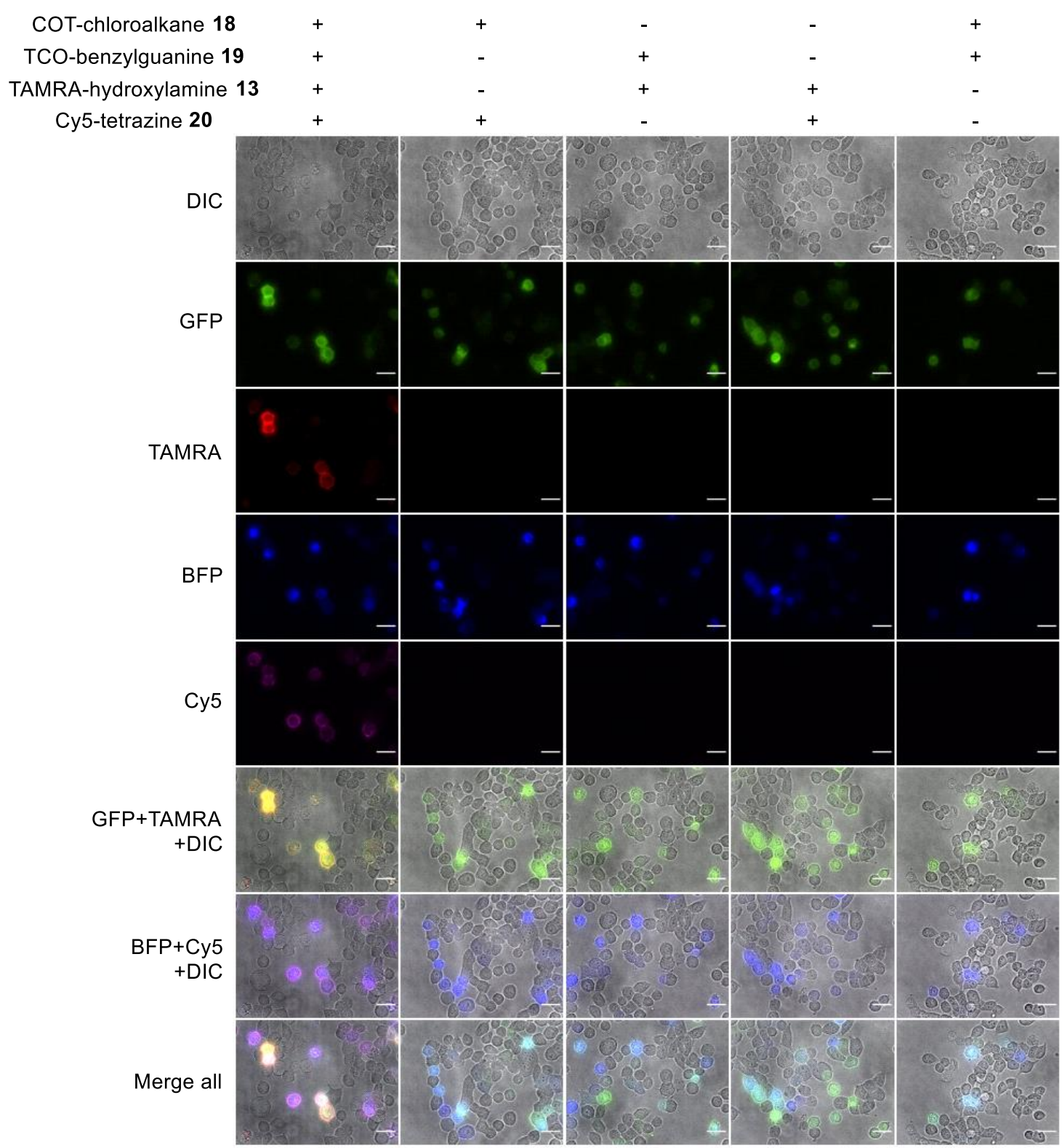

Figure S9. Live cell dual labeling with orthogonal cyclooctyne-hydroxylamine and transcyclooctenetetrazine ligations. Scale bar $=25 \mu \mathrm{m}$. 


\section{Intracellular Labeling Experiment}

pHTC-GFP-HaloTag-His6:

ATGGTTAGCAAAGGTGAAGAACTGTTTACCGGCGTTGTGCCGATTCTGGTGGAACTGGA TGGTGATGTGAATGGCCATAAATTTAGCGTTCGTGGCGAAGGCGAAGGTGATGCGACCA ACGGTAAACTGACCCTGAAATTTATTTGCACCACCGGTAAACTGCCGGTTCCGTGGCCGA CCCTGGTGACCACCCTGACCTATGGCGTTCAGTGCTTTAGCCGCTATCCGGATCATATGA AACGCCATGATTTCTTTAAAAGCGCGATGCCGGAAGGCTATGTGCAGGAACGTACCATT AGCTTCAAAGATGATGGCACCTATAAAACCCGTGCGGAAGTTAAATTTGAAGGCGATAC CCTGGTGAACCGCATTGAACTGAAAGGTATTGATTTTAAAGAAGATGGCAACATTCTGG GTCATAAACTGGAATATAATTTCAACAGCCATGCGGTGTATATTACCGCCGATAAACAG AAAAATGGCATCAAAGCGAACTTTAAAATCCGTCACAACGTGGAAGATGGTAGCGTGCA GCTGGCGGATCATTATCAGCAGAATACCCCGATTGGTGATGGCCCGGTGCTGCTGCCGG ATAATCATTATCTGAGCACCCAGAGCGTTCTGAGCAAAGATCCGAATGAAAAACGTGAT CATATGGTGCTGCTGGAATTTGTTACCGCCGCGGGCATTACCCACGGTATGGATGAACTG TATAAAGGCAGCCACCATCATCATCACCATCTCGAGCCAACCACTGAGGATCTGTACTTT CAGAGCGATAACGATGGATCCGAAATCGGTACTGGCTTTCCATTCGACCCCCATTATGTG GAAGTCCTGGGCGAGCGCATGCACTACGTCGATGTTGGTCCGCGCGATGGCACCCCTGT GCTGTTCCTGCACGGTAACCCGACCTCCTCCTACGTGTGGCGCAACATCATCCCGCATGT TGCACCGACCCATCGCTGCATTGCTCCAGACCTGATCGGTATGGGCAAATCCGACAAAC CAGACCTGGGTTATTTCTTCGACGACCACGTCCGCTTCATGGATGCCTTCATCGAAGCCC TGGGTCTGGAAGAGGTCGTCCTGGTCATTCACGACTGGGGCTCCGCTCTGGGTTTCCACT GGGCCAAGCGCAATCCAGAGCGCGTCAAAGGTATTGCATTTATGGAGTTCATCCGCCCT ATCCCGACCTGGGACGAATGGCCAGAATTTGCCCGCGAGACCTTCCAGGCCTTCCGCAC CACCGACGTCGGCCGCAAGCTGATCATCGATCAGAACGTTTTTATCGAGGGTACGCTGC CGATGGGTGTCGTCCGCCCGCTGACTGAAGTCGAGATGGACCATTACCGCGAGCCGTTC CTGAATCCTGTTGACCGCGAGCCACTGTGGCGCTTCCCAAACGAGCTGCCAATCGCCGGT GAGCCAGCGAACATCGTCGCGCTGGTCGAAGAATACATGGACTGGCTGCACCAGTCCCC TGTCCCGAAGCTGCTGTTCTGGGGCACCCCAGGCGTTCTGATCCCACCGGCCGAAGCCGC TCGCCTGGCCAAAAGCCTGCCTAACTGCAAGGCTGTGGACATCGGCCCGGGTCTGAATC TGCTGCAAGAAGACAACCCGGACCTGATCGGCAGCGAGATCGCGCGCTGGCTGTCTACT CTGGAGATTTCCGGTCATCATCATCACCACCAC

Preparation for 12-Well Plate: Coverslips (15 mm circle, thickness $0.13-0.17 \mathrm{~mm}$ ) were acid-washed according to the Cold Spring Harbor protocol. ${ }^{6}$ A coverslip was added to each well in a 12-well plate followed by an aqueous solution of poly-D-lysine $(0.1 \mathrm{mg} / \mathrm{mL}, 30-70 \mathrm{kDa}, 800 \mu \mathrm{L})$. The plate was gently shaken to distribute the solution evenly, incubated at room temperature for $1 \mathrm{~h}$, washed using 1 $\mathrm{mL}$ of autoclaved deionized water $(1 \mathrm{~mL})$ and $\mathrm{PBS}(\mathrm{pH} 7.4,1 \mathrm{~mL})$, and dried.

Intracellular labeling experiment: HeLa cells were seeded at a density of 200,000 cells per well in 1 $\mathrm{mL}$ of RPMI with $10 \%$ FBS (Sigma), 100 units $/ \mathrm{mL}$ penicillin, and $0.1 \mathrm{mg} / \mathrm{mL}$ streptomycin (Sigma). Cells were then grown in a humidified chamber at $37{ }^{\circ} \mathrm{C}$ with $5 \% \mathrm{CO}_{2}$. Upon reaching $\sim 80 \%$ confluency, the cells in each well were transfected with Mirus Bio TransIT-LT1 transfection reagent $(3 \mu \mathrm{L})$ and plasmid $(1.0 \mu \mathrm{g}$ of pHTC-GFP-HaloTag-His6) in OptiMEM I reduced serum medium $(100 \mu \mathrm{L})$. After $36 \mathrm{~h}$, cells were washed with PBS $(3 \times 1 \mathrm{~mL})$. Serum-free RPMI $(400 \mu \mathrm{L})$ was added to each well containing compounds $(10 \mu \mathrm{M})$ or vehicle (DMSO) as follows: 
Well 1 and 2: COT-chloroalkane $18(2.00 \mu \mathrm{L}, 2 \mathrm{mM}$ stock solution in DMSO; final concentration 10 $\mu \mathrm{M})$ in serum-free RPMI $(400 \mu \mathrm{L})$

Well 3 and 4: DMSO $(2.00 \mu \mathrm{L})$ in serum-free RMPI $(400 \mu \mathrm{L})$

Cells were incubated at $37{ }^{\circ} \mathrm{C}$ for $30 \mathrm{~min}$. After incubation, cells were washed with PBS $(3 \times 1 \mathrm{~mL})$. Then, serum-free RPMI $(400 \mu \mathrm{L})$ was added to each well containing fluorophore $(50 \mu \mathrm{M})$ or vehicle (autoclaved deionized water) as follows:

Well 1 and 3: TAMRA-hydroxylamine $13\left(2.00 \mu \mathrm{L}, 10 \mathrm{mM}\right.$ stock solution in $\mathrm{H}_{2} \mathrm{O}$; final concentration $50 \mu \mathrm{M})$ in serum-free RPMI $(400 \mu \mathrm{L})$

Well 2 and 4: autoclaved deionized water $(2.00 \mu \mathrm{L})$ in serum-free RPMI $(400 \mu \mathrm{L})$

Cells were incubated at $37{ }^{\circ} \mathrm{C}$ for $1 \mathrm{~h}$. After incubation, cells were washed with PBS. Paraformaldehyde solution ( $4 \% \mathrm{w} / \mathrm{v}$ in $\mathrm{H}_{2} \mathrm{O}, 1 \mathrm{~mL}$ ) was then added to fix the cells, and the solution was incubated at room temperature for $20 \mathrm{~min}$. After gently washing the cells with PBS $(3 \times 1 \mathrm{~mL})$, each coverslip was lifted, washed by dipping twice into deionized water in a $200 \mathrm{~mL}$ beaker, and mounted on a microscopy slide using aqueous mounting media (20 mM Tris pH 8.0, 0.5\% N-propyl gallate, $90 \%$ glycerol).

Fluorescence microscopy: Slides were imaged at the Confocal and Light Microscopy Core at DanaFarber Cancer Institute using a Nikon Ti Eclipse. Images were acquired with a 40× objective at 0.16 micron/pixel using a Hamamatsu camera. GFP was imaged with a 482/35 filter (excitation) and a 536/75 filter (emission) and false-colored green and TAMRA was imaged with a 560/40 filter (excitation) and a 630/75 filter (emission) and false-colored red. Images were processed with Fiji ImageJ software.

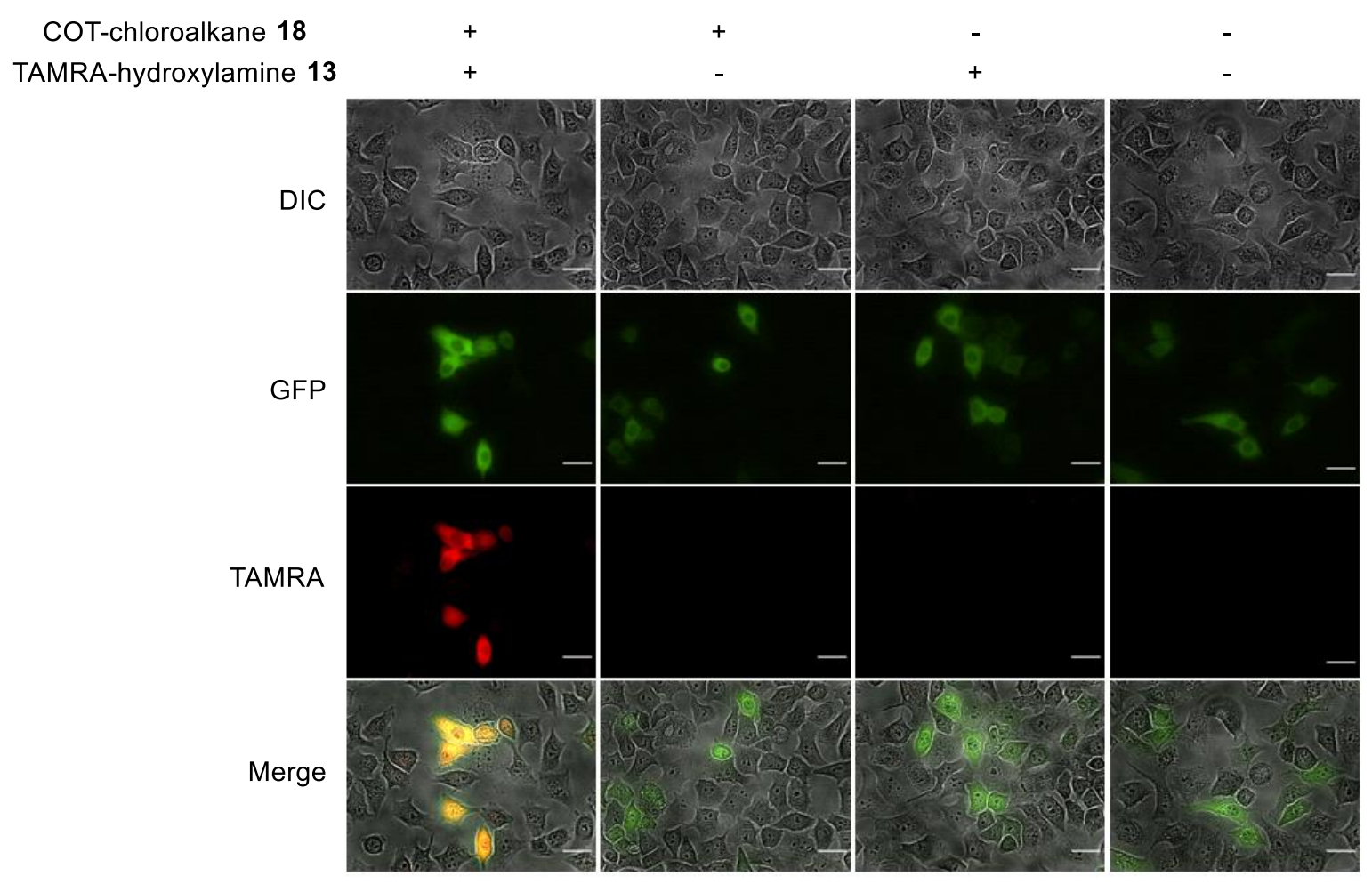

Figure S10. Intracellular protein labeling with cyclooctyne 18 and TAMRA-hydroxylamine 13. Scale bar $=25 \mu \mathrm{m}$. 


\section{Cell Viability Assay}

HeLa cells were seeded in an opaque 96-well plate at a density of 12,000 cells per well in media (100 $\mu \mathrm{L}$, PRMI containing $10 \% \mathrm{FBS}, 100$ units/mL penicillin, $0.1 \mathrm{mg} / \mathrm{mL}$ streptomycin). PBS $(100 \mu \mathrm{L})$ was added to the edge wells. The cells were incubated in a humidified chamber at $37{ }^{\circ} \mathrm{C}$ with $5 \%$ $\mathrm{CO}_{2}$. After $24 \mathrm{~h}$, the media was aspirated and replaced with media $(50 \mu \mathrm{L}$, RPMI supplemented with $10 \% \mathrm{FBS}, 100$ units/mL penicillin, $0.1 \mathrm{mg} / \mathrm{mL}$ streptomycin) containing hydroxylamine 13.

Treatment concentrations started at $50 \mu \mathrm{M}$ and was serially diluted 3-fold across nine wells. The plate was incubated in a humidified chamber at $37{ }^{\circ} \mathrm{C}$ with $5 \% \mathrm{CO}_{2}$. After $24 \mathrm{~h}$, the plate was equilibrated to room temperature. CellTiter-Glo reagent ( $50 \mu \mathrm{L}$, Promega, prepared by manufacturer's protocol) was added to each well and mixed gently. The plate was incubated at room temperature for $10 \mathrm{~min}$ to stabilize the luminescence signal. The absorbance at $570 \mathrm{~nm}$ was measured using a microplate reader (Clariostar Plus, BMG Labtech) to give $\mathrm{IC}_{50}$ value $>50 \mu \mathrm{M}$.

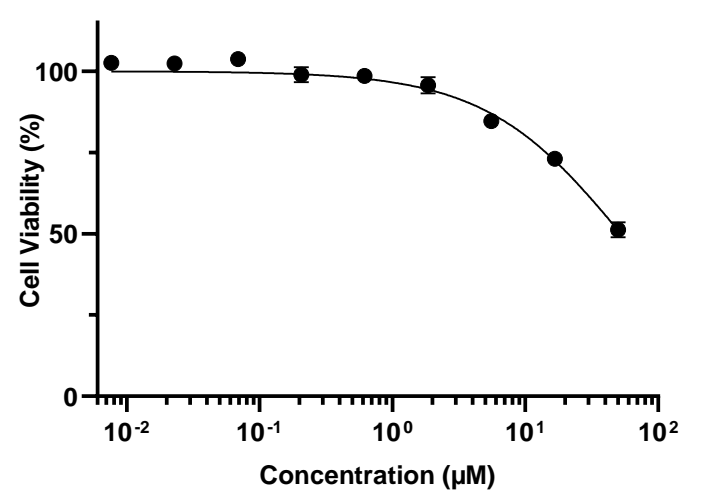

Figure S11. Dose response cell viability curves for HeLa cells. Cells were treated with hydroxylamine 13 for $24 \mathrm{~h}$. Error bars represent mean \pm SEM of data from biological replicates $(n=$ 4). 


\section{Computational Details}

All calculations were conducted with Gaussian 09 software. ${ }^{7}$ Geometry optimization of all species was performed using the M06-2X functional ${ }^{8}$ with the 6-31G(d) basis set. Frequency analysis was carried out to ensure the stationary point was either a minimum or a transition state. Intrinsic reaction coordinates were computed for all transition states. Single-point calculations were carried out using the M06-2X functional with the 6-311G(2d,p) basis set. For each cyclooctyne, at least three different conformers were analyzed via geometry optimization and the most stable conformation was adapted to locate the transition states. The 3D image in Figure $2 \mathrm{~B}$ was generated by using CYLview. ${ }^{9}$

- Cartesian coordinates of optimized structures $(\AA)$

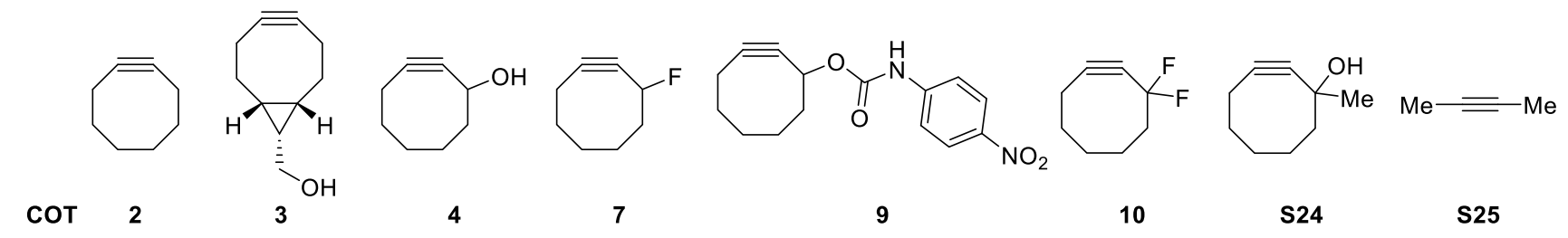

2

Lowest three frequencies $\left(\mathrm{cm}^{-1}\right): 110.03,162.71,241.84$

$\mathrm{E}(\mathrm{RM} 062 \mathrm{X})=-311.941223533$

$\begin{array}{lrrr}\mathrm{C} & -0.68292200 & 1.34395100 & -0.37416000 \\ \mathrm{C} & 0.68329400 & 1.34396600 & 0.37395400 \\ \mathrm{C} & -0.60303500 & -1.45391500 & -0.03049400 \\ \mathrm{C} & 1.85549200 & 0.58004700 & -0.28167000 \\ \mathrm{C} & 0.60265200 & -1.45397600 & 0.02990500 \\ \mathrm{C} & 1.95689300 & -0.90982700 & 0.12276300 \\ \mathrm{H} & -0.54219700 & 0.96955700 & -1.39589000 \\ \mathrm{H} & 0.54250900 & 0.96977100 & 1.39573900 \\ \mathrm{H} & 1.75673900 & 0.64014800 & -1.37149500 \\ \mathrm{H} & -1.00069900 & 2.38614400 & -0.48058600 \\ \mathrm{H} & 1.00126400 & 2.38613400 & 0.48016800 \\ \mathrm{H} & 2.80150000 & 1.06939900 & -0.02463200 \\ \mathrm{H} & 2.32183800 & -0.99477600 & 1.15300500 \\ \mathrm{C} & -1.85519100 & 0.58044700 & 0.28178400 \\ \mathrm{H} & -2.80118600 & 1.06994000 & 0.02495600 \\ \mathrm{H} & -1.75621700 & 0.64055900 & 1.37159300 \\ \mathrm{C} & -1.95726000 & -0.90951700 & -0.12239500 \\ \mathrm{H} & -2.32324900 & -0.99453800 & -1.15225700 \\ \mathrm{H} & -2.67291700 & -1.43432800 & 0.51762800 \\ \mathrm{H} & 2.67307900 & -1.43506700 & -0.51634600\end{array}$

\section{2-TS}

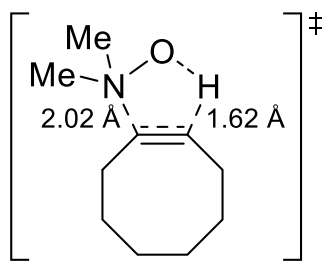


Lowest three frequencies $\left(\mathrm{cm}^{-1}\right):-210.84,54.59,63.58$ $\mathrm{E}(\mathrm{RM} 062 \mathrm{X})=-597.454128075$

$\begin{array}{lrrr}\mathrm{C} & -2.66445400 & -1.33159700 & 0.25384500 \\ \mathrm{C} & -2.70860600 & 0.11134700 & 0.77854500 \\ \mathrm{C} & 0.23405900 & -0.01687700 & -0.10192300 \\ \mathrm{C} & -2.62269200 & 1.20627700 & -0.30455500 \\ \mathrm{C} & -0.10056100 & 1.18988900 & -0.16109100 \\ \mathrm{C} & -1.32770700 & 2.03024300 & -0.20765300 \\ \mathrm{H} & -3.59000700 & -1.53272000 & -0.30043300 \\ \mathrm{H} & -1.89403400 & 0.25541200 & 1.49723700 \\ \mathrm{H} & -2.67496500 & 0.75304900 & -1.30180100 \\ \mathrm{H} & -2.67237900 & -2.01222300 & 1.11501700 \\ \mathrm{H} & -3.63774000 & 0.22238000 & 1.34753100 \\ \mathrm{H} & -3.48153200 & 1.88284300 & -0.23173900 \\ \mathrm{H} & -1.35646000 & 2.64314400 & 0.70289600 \\ \mathrm{C} & -1.49155500 & -1.71260800 & -0.65786900 \\ \mathrm{H} & -1.56283600 & -1.17719800 & -1.61096600 \\ \mathrm{H} & -1.58407100 & -2.77893300 & -0.89383500 \\ \mathrm{C} & -0.08708600 & -1.46293400 & -0.08011000 \\ \mathrm{H} & 0.64290000 & -2.03036700 & -0.66907100 \\ \mathrm{H} & -0.03127100 & -1.85344000 & 0.94630500 \\ \mathrm{H} & -1.26789200 & 2.74388800 & -1.03715400 \\ \mathrm{H} & 1.42967300 & 1.69564600 & -0.00213800 \\ \mathrm{~N} & 2.24740000 & 0.04409500 & 0.06739800 \\ \mathrm{C} & 2.67445500 & -0.55729400 & 1.32517900 \\ \mathrm{H} & 2.47108700 & -1.63102300 & 1.29984500 \\ \mathrm{H} & 3.74417800 & -0.38817100 & 1.49393400 \\ \mathrm{H} & 2.10026100 & -0.09070700 & 2.12625200 \\ \mathrm{C} & 2.91198200 & -0.49989900 & -1.11211100 \\ \mathrm{H} & 3.98469800 & -0.27849500 & -1.08307300 \\ \mathrm{H} & 2.76495000 & -1.58165900 & -1.15333500 \\ \mathrm{H} & 2.46586200 & -0.03045300 & -1.98961400 \\ \mathrm{O} & 2.43884600 & 1.39263400 & 0.10985100\end{array}$

3

Lowest three frequencies $\left(\mathrm{cm}^{-1}\right): 76.84,119.98,150.60$ $\mathrm{E}(\mathrm{RM} 062 \mathrm{X})=-464.533804304$

$\begin{array}{lrrr}\mathrm{C} & -0.01283500 & -1.00254400 & -0.80085300 \\ \mathrm{C} & 0.37899600 & 0.45714600 & -0.91815300 \\ \mathrm{C} & -2.50601300 & -0.06311900 & 0.26412800 \\ \mathrm{C} & 0.17822400 & 1.53774600 & 0.13058700 \\ \mathrm{C} & -2.17464500 & 1.09620100 & 0.18957400 \\ \mathrm{C} & -1.22089500 & 2.19913000 & 0.04245500 \\ \mathrm{H} & 0.29307700 & 1.12838000 & 1.13911500 \\ \mathrm{H} & 0.96102000 & 2.29404100 & 0.00591900 \\ \mathrm{H} & -1.33393400 & 2.96063000 & 0.82004300 \\ \mathrm{H} & -1.34715300 & 2.70086200 & -0.92366200 \\ \mathrm{C} & -0.72754500 & -1.66251700 & 0.36754800 \\ \mathrm{H} & -0.40894300 & -1.23089700 & 1.32086800\end{array}$




$\begin{array}{lrrr}\mathrm{H} & -0.46644200 & -2.72809700 & 0.39078900 \\ \mathrm{C} & -2.26809700 & -1.50908400 & 0.28252000 \\ \mathrm{H} & -2.74729900 & -2.00550200 & 1.13185500 \\ \mathrm{H} & -2.65066000 & -1.98299900 & -0.62864600 \\ \mathrm{C} & 1.44406000 & -0.60117600 & -0.78352100 \\ \mathrm{H} & 2.02067800 & -0.81919500 & -1.67846400 \\ \mathrm{H} & -0.33231800 & -1.42929600 & -1.75073000 \\ \mathrm{H} & 0.26603600 & 0.84783700 & -1.92828500 \\ \mathrm{C} & 2.27399100 & -0.66775500 & 0.46982800 \\ \mathrm{H} & 2.81936200 & -1.62295200 & 0.49704300 \\ \mathrm{H} & 1.63764000 & -0.63371900 & 1.36438300 \\ \mathrm{O} & 3.16866000 & 0.43112800 & 0.44543400 \\ \mathrm{H} & 3.74821600 & 0.36770100 & 1.21162500\end{array}$

\section{3-TS}

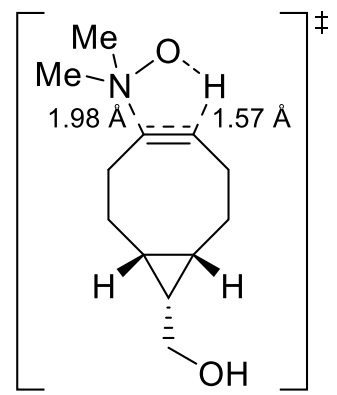

Lowest three frequencies $\left(\mathrm{cm}^{-1}\right)$ : $-258.31,39.54,58.07$ $\mathrm{E}(\mathrm{RM} 062 \mathrm{X})=-674.832028183$

$\begin{array}{lrrr}\mathrm{C} & 1.85584600 & 1.06409700 & 0.78212800 \\ \mathrm{C} & 1.62694400 & -0.41961300 & 0.91973700 \\ \mathrm{C} & -1.04885100 & 1.27280900 & -0.19321700 \\ \mathrm{C} & 0.98383100 & -1.26256600 & -0.15990500 \\ \mathrm{C} & -1.14074600 & 0.02091800 & -0.10622200 \\ \mathrm{C} & -0.54380500 & -1.33232900 & -0.00964000 \\ \mathrm{H} & 1.22044700 & -0.87404600 & -1.15497900 \\ \mathrm{H} & 1.40065900 & -2.27447500 & -0.11594200 \\ \mathrm{H} & -0.95309500 & -1.99803500 & -0.77924300 \\ \mathrm{H} & -0.78749500 & -1.78685000 & 0.96036800 \\ \mathrm{C} & 1.41279300 & 1.86504500 & -0.42404200 \\ \mathrm{H} & 1.48590100 & 1.27450200 & -1.34237400 \\ \mathrm{H} & 2.08124200 & 2.72700200 & -0.54972500 \\ \mathrm{C} & -0.03588500 & 2.35807800 & -0.28617200 \\ \mathrm{H} & -0.27924100 & 3.01464000 & -1.12973000 \\ \mathrm{H} & -0.12165500 & 2.99189500 & 0.60659000 \\ \mathrm{C} & 3.02875000 & 0.11200100 & 0.77360300 \\ \mathrm{H} & 3.63480200 & 0.09044100 & 1.67524700 \\ \mathrm{H} & 1.74518500 & 1.62357000 & 1.70963600 \\ \mathrm{H} & 1.36736400 & -0.75253000 & 1.92312200 \\ \mathrm{C} & 3.82436500 & -0.17914900 & -0.46937700 \\ \mathrm{H} & 4.72265600 & 0.45584100 & -0.48417000 \\ \mathrm{H} & 3.24564900 & 0.06069500 & -1.37137600\end{array}$




$\begin{array}{lrrr}\mathrm{O} & 4.17246200 & -1.55384200 & -0.44345500 \\ \mathrm{H} & 4.74174300 & -1.73555900 & -1.19835200 \\ \mathrm{H} & -2.60870700 & 1.47160700 & -0.14425100 \\ \mathrm{~N} & -3.09562800 & -0.30309800 & -0.01817100 \\ \mathrm{C} & -3.56241300 & -1.04259200 & -1.18780100 \\ \mathrm{H} & -3.19063300 & -2.06896000 & -1.14779700 \\ \mathrm{H} & -4.65713500 & -1.04908900 & -1.22039300 \\ \mathrm{H} & -3.17289900 & -0.53892700 & -2.07311300 \\ \mathrm{C} & -3.47059100 & -0.90818400 & 1.25669400 \\ \mathrm{H} & -4.56019000 & -0.91712300 & 1.36816900 \\ \mathrm{H} & -3.08753900 & -1.93024900 & 1.30381600 \\ \mathrm{H} & -3.02642200 & -0.30672500 & 2.05058300 \\ \mathrm{O} & -3.56154500 & 0.97296200 & -0.07324700\end{array}$

\section{4}

Lowest three frequencies $\left(\mathrm{cm}^{-1}\right): 88.35,145.59,170.29$ $\mathrm{E}(\mathrm{RM} 062 \mathrm{X})=-387.155738469$

$\begin{array}{lrrr}\mathrm{C} & -1.40444800 & 1.16077100 & -0.45525300 \\ \mathrm{C} & -0.15369900 & 1.58367700 & 0.37115600 \\ \mathrm{C} & -0.55528900 & -1.47041500 & 0.07093300 \\ \mathrm{C} & 1.22920300 & 1.15210200 & -0.15836200 \\ \mathrm{C} & 0.60308800 & -1.14617500 & 0.18041400 \\ \mathrm{C} & 1.72348700 & -0.20838500 & 0.36079200 \\ \mathrm{H} & -1.08845000 & 0.80277200 & -1.44282500 \\ \mathrm{H} & -0.26482200 & 1.23800300 & 1.40698100 \\ \mathrm{H} & 1.21662800 & 1.08668700 & -1.25184400 \\ \mathrm{H} & -1.99693200 & 2.06216200 & -0.64089700 \\ \mathrm{H} & -0.14809000 & 2.67654700 & 0.42585000 \\ \mathrm{H} & 1.98863200 & 1.89489700 & 0.10777200 \\ \mathrm{H} & 1.93435700 & -0.12372800 & 1.43908100 \\ \mathrm{C} & -2.35600600 & 0.12050400 & 0.17627500 \\ \mathrm{H} & -3.37963800 & 0.29968800 & -0.17009600 \\ \mathrm{H} & -2.36437800 & 0.25488400 & 1.26382800 \\ \mathrm{C} & -1.99639300 & -1.35254800 & -0.13627700 \\ \mathrm{H} & -2.23863400 & -1.58396400 & -1.17979900 \\ \mathrm{O} & 2.90095600 & -0.52443600 & -0.35070800 \\ \mathrm{H} & -2.57669200 & -2.03460500 & 0.49195500 \\ \mathrm{H} & 3.17071800 & -1.41504200 & -0.10241500\end{array}$

\section{4-TS}

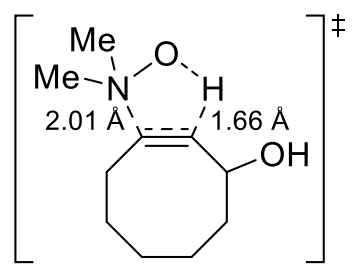

Lowest three frequencies $\left(\mathrm{cm}^{-1}\right):-182.60,48.39,53.20$ $\mathrm{E}(\mathrm{RM} 062 \mathrm{X})=-597.454857190$ C $\quad 2.08255600 \quad-1.79648500 \quad 0.50416900$ 


$\begin{array}{lrrr}\mathrm{C} & 3.00686600 & -0.60652000 & 0.17522800 \\ \mathrm{C} & -0.39646100 & -0.30639400 & -0.25881200 \\ \mathrm{C} & 2.53293300 & 0.81360200 & 0.50680400 \\ \mathrm{C} & 0.17386700 & 0.80649400 & -0.30114300 \\ \mathrm{C} & 1.50508500 & 1.44094700 & -0.44640700 \\ \mathrm{H} & 1.54248400 & -1.58854500 & 1.43749800 \\ \mathrm{H} & 3.29050300 & -0.65447100 & -0.88525800 \\ \mathrm{H} & 2.12658000 & 0.84187300 & 1.52703400 \\ \mathrm{H} & 2.73013100 & -2.65278000 & 0.71845400 \\ \mathrm{H} & 3.93735500 & -0.75727000 & 0.73362400 \\ \mathrm{H} & 3.40093100 & 1.48157800 & 0.49285600 \\ \mathrm{H} & 1.85519100 & 1.29840300 & -1.47741300 \\ \mathrm{C} & 1.08112500 & -2.23005700 & -0.58633000 \\ \mathrm{H} & 1.07999600 & -3.32178900 & -0.67386800 \\ \mathrm{H} & 1.40009400 & -1.83936800 & -1.55966400 \\ \mathrm{C} & -0.36287800 & -1.77946800 & -0.32834500 \\ \mathrm{H} & -0.71887500 & -2.22049600 & 0.61249200 \\ \mathrm{O} & 1.47488800 & 2.85025700 & -0.26426300 \\ \mathrm{H} & -1.01750800 & -2.15439800 & -1.12448300 \\ \mathrm{H} & 0.92877100 & 3.01758800 & 0.51218300 \\ \mathrm{H} & -1.25869700 & 1.61496200 & -0.05276500 \\ \mathrm{~N} & -2.33096700 & 0.12430200 & 0.11897700 \\ \mathrm{C} & -2.73729100 & -0.36679100 & 1.43113600 \\ \mathrm{H} & -3.71547700 & 0.04094000 & 1.70898000 \\ \mathrm{H} & -2.78670300 & -1.45794900 & 1.41167400 \\ \mathrm{H} & -1.98711300 & -0.04247600 & 2.15367600 \\ \mathrm{C} & -3.19730300 & -0.30361200 & -0.97438400 \\ \mathrm{H} & -3.23588700 & -1.39513400 & -1.00105200 \\ \mathrm{H} & -4.20854100 & 0.09728100 & -0.84384400 \\ \mathrm{H} & -2.77091100 & 0.07593100 & -1.90368900 \\ \mathrm{O} & -2.27570700 & 1.48920700 & 0.14541600\end{array}$

\section{7}

Lowest three frequencies $\left(\mathrm{cm}^{-1}\right): 88.56,145.94,170.90$ $\mathrm{E}(\mathrm{RM} 062 \mathrm{X})=-411.178599922$

$\begin{array}{lrrr}\mathrm{C} & -1.38416000 & 1.17714000 & -0.44941600 \\ \mathrm{C} & -0.12115600 & 1.58443800 & 0.36548100 \\ \mathrm{C} & -0.56314300 & -1.46906000 & 0.06275800 \\ \mathrm{C} & 1.25205200 & 1.12418600 & -0.16592300 \\ \mathrm{C} & 0.60291400 & -1.17376500 & 0.16709000 \\ \mathrm{C} & 1.71425700 & -0.24071200 & 0.36059400 \\ \mathrm{H} & -1.08281100 & 0.82163800 & -1.44247400 \\ \mathrm{H} & -0.23343700 & 1.25285000 & 1.40583200 \\ \mathrm{H} & 1.23256600 & 1.05238500 & -1.25883900 \\ \mathrm{H} & -1.96802900 & 2.08619500 & -0.62363400 \\ \mathrm{H} & -0.09415300 & 2.67726600 & 0.40855400 \\ \mathrm{H} & 2.02438100 & 1.85539600 & 0.09452300 \\ \mathrm{H} & 1.96673200 & -0.17605900 & 1.42663400 \\ \mathrm{C} & -2.34093300 & 0.14522000 & 0.18740100\end{array}$




$\begin{array}{lrrr}H & -3.36537300 & 0.33795000 & -0.14849400 \\ \mathrm{H} & -2.33777300 & 0.27564000 & 1.27541600 \\ \mathrm{C} & -2.00307400 & -1.33077300 & -0.13423800 \\ \mathrm{H} & -2.25340600 & -1.55450000 & -1.17742400 \\ \mathrm{H} & -2.58721000 & -2.00872000 & 0.49456300 \\ \mathrm{~F} & 2.86199800 & -0.61334300 & -0.31301600\end{array}$

\section{7-TS}

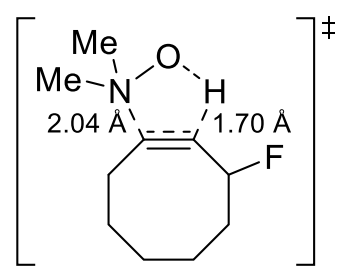

Lowest three frequencies $\left(\mathrm{cm}^{-1}\right):-148.74,47.84,53.77$ $\mathrm{E}(\mathrm{RM} 062 \mathrm{X})=-621.4807673$

$\begin{array}{lrrr}\mathrm{C} & 2.09957800 & -1.77336700 & 0.50460200 \\ \mathrm{C} & 3.01413400 & -0.58224700 & 0.14693100 \\ \mathrm{C} & -0.38454600 & -0.30961600 & -0.25940900 \\ \mathrm{C} & 2.54526100 & 0.83632400 & 0.49176100 \\ \mathrm{C} & 0.18142700 & 0.80059500 & -0.29535300 \\ \mathrm{C} & 1.49617300 & 1.43441900 & -0.44107000 \\ \mathrm{H} & 1.56875600 & -1.55399600 & 1.44030900 \\ \mathrm{H} & 3.27029300 & -0.63251200 & -0.92038700 \\ \mathrm{H} & 2.15375100 & 0.86941500 & 1.51495300 \\ \mathrm{H} & 2.75601900 & -2.62099800 & 0.72545600 \\ \mathrm{H} & 3.95914000 & -0.72898600 & 0.68068600 \\ \mathrm{H} & 3.40828000 & 1.51022900 & 0.45742300 \\ \mathrm{H} & 1.83260100 & 1.34662500 & -1.48390800 \\ \mathrm{C} & 1.08899200 & -2.23525000 & -0.56665100 \\ \mathrm{H} & 1.09042100 & -3.32878100 & -0.62654600 \\ \mathrm{H} & 1.39955200 & -1.86880400 & -1.55210600 \\ \mathrm{C} & -0.35524900 & -1.78211700 & -0.30890700 \\ \mathrm{H} & -0.70688800 & -2.20615300 & 0.64103300 \\ \mathrm{H} & -1.01480600 & -2.16802100 & -1.09549000 \\ \mathrm{~F} & 1.42927700 & 2.79903800 & -0.16126700 \\ \mathrm{H} & -1.27552600 & 1.62894100 & -0.00695500 \\ \mathrm{~N} & -2.34687500 & 0.12478000 & 0.11474500 \\ \mathrm{C} & -2.77268100 & -0.38105100 & 1.41444700 \\ \mathrm{H} & -3.75140100 & 0.02912900 & 1.68799100 \\ \mathrm{H} & -2.82990700 & -1.47157600 & 1.37792800 \\ \mathrm{H} & -2.02964300 & -0.07311500 & 2.15154900 \\ \mathrm{C} & -3.20662000 & -0.27556600 & -0.99287400 \\ \mathrm{H} & -3.25266800 & -1.36634100 & -1.03924600 \\ \mathrm{H} & -4.21700100 & 0.12969700 & -0.86620700 \\ \mathrm{H} & -2.76937400 & 0.11616800 & -1.91213100 \\ \mathrm{O} & -2.28322100 & 1.49144100 & 0.16412200 \\ & & & \\ & & & \end{array}$


9

Lowest three frequencies $\left(\mathrm{cm}^{-1}\right): 18.78,27.04,38.19$

$\mathrm{E}(\mathrm{RM} 062 \mathrm{X})=-991.375222368$

$\begin{array}{lrrr}\mathrm{C} & -5.69733200 & -0.78406700 & 1.08409800 \\ \mathrm{C} & -4.49468400 & -1.57956700 & 0.49576700 \\ \mathrm{C} & -4.90559600 & 1.25176100 & -0.68990800 \\ \mathrm{C} & -3.08468900 & -0.97571000 & 0.66702000 \\ \mathrm{C} & -3.75135300 & 0.89924200 & -0.70423300 \\ \mathrm{C} & -2.65813600 & -0.04137300 & -0.47976300 \\ \mathrm{H} & -5.32793700 & -0.00240700 & 1.75949200 \\ \mathrm{H} & -4.67113200 & -1.78095800 & -0.56835000 \\ \mathrm{H} & -3.03321400 & -0.39610900 & 1.59560800 \\ \mathrm{H} & -6.26973200 & -1.47314900 & 1.71251300 \\ \mathrm{H} & -4.47688100 & -2.56057400 & 0.97935300 \\ \mathrm{H} & -2.33689600 & -1.77207600 & 0.73007000 \\ \mathrm{H} & -2.45311400 & -0.62634500 & -1.38224900 \\ \mathrm{C} & -6.69320500 & -0.15646100 & 0.08371400 \\ \mathrm{H} & -7.69278000 & -0.12580200 & 0.52990600 \\ \mathrm{H} & -6.76450600 & -0.79655900 & -0.80266900 \\ \mathrm{C} & -6.33006000 & 1.27840900 & -0.37078700 \\ \mathrm{H} & -6.50965600 & 1.98750400 & 0.44513800 \\ \mathrm{O} & -1.45648400 & 0.66859500 & -0.12703200 \\ \mathrm{C} & -0.31126200 & -0.01446800 & -0.31068200 \\ \mathrm{O} & -0.23782400 & -1.14473000 & -0.73518700 \\ \mathrm{~N} & 0.73863700 & 0.78883300 & 0.06172300 \\ \mathrm{H} & 0.47560600 & 1.70972100 & 0.38282900 \\ \mathrm{C} & 2.09962300 & 0.47965600 & 0.03985300 \\ \mathrm{C} & 2.98476800 & 1.47608600 & 0.48070200 \\ \mathrm{C} & 2.59995000 & -0.75667900 & -0.39452300 \\ \mathrm{C} & 4.34940400 & 1.25241800 & 0.49287500 \\ \mathrm{H} & 2.59305700 & 2.43196600 & 0.81553600 \\ \mathrm{C} & 3.96933600 & -0.97923300 & -0.38116000 \\ \mathrm{H} & 1.92005900 & -1.52329900 & -0.73570100 \\ \mathrm{C} & 4.82453600 & 0.02090500 & 0.05969100 \\ \mathrm{H} & 5.04783300 & 2.00812600 & 0.82919500 \\ \mathrm{H} & 4.38446200 & -1.92356700 & -0.71065800 \\ \mathrm{~N} & 6.26742300 & -0.22515200 & 0.06949500 \\ \mathrm{O} & 6.65732000 & -1.31206600 & -0.31685700 \\ \mathrm{O} & 6.98895800 & 0.67374800 & 0.46426200 \\ \mathrm{H} & -6.95114700 & 1.58788100 & -1.21601000\end{array}$

\section{9-TS}

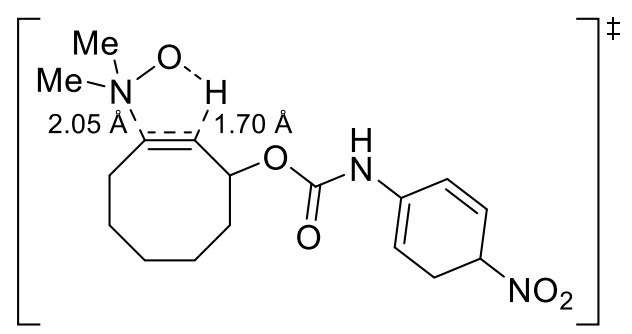


Lowest three frequencies $\left(\mathrm{cm}^{-1}\right):-136.00,17.92,22.89$ $\mathrm{E}(\mathrm{RM} 062 \mathrm{X})=-1201.67730053$

$\begin{array}{lrrr}\mathrm{C} & -4.38614700 & 2.68512900 & -0.67086600 \\ \mathrm{C} & -2.88110800 & 2.98010600 & -0.49513100 \\ \mathrm{C} & -4.09571100 & -0.12491600 & 0.27992600 \\ \mathrm{C} & -1.86372600 & 1.90456800 & -0.89809500 \\ \mathrm{C} & -2.85392700 & -0.12269900 & 0.17417500 \\ \mathrm{C} & -1.69063300 & 0.76355400 & 0.10627800 \\ \mathrm{H} & -4.53137800 & 2.04868700 & -1.55375500 \\ \mathrm{H} & -2.68832400 & 3.28969400 & 0.54075400 \\ \mathrm{H} & -2.12962700 & 1.47280000 & -1.86988900 \\ \mathrm{H} & -4.86909900 & 3.63810800 & -0.90835000 \\ \mathrm{H} & -2.64975400 & 3.85708500 & -1.10865800 \\ \mathrm{H} & -0.88620600 & 2.38615700 & -1.00372100 \\ \mathrm{H} & -1.46421700 & 1.17942000 & 1.09441900 \\ \mathrm{C} & -5.13253100 & 2.07538200 & 0.53407600 \\ \mathrm{H} & -6.10218100 & 2.56965900 & 0.65462900 \\ \mathrm{H} & -4.56881100 & 2.26626000 & 1.45456200 \\ \mathrm{C} & -5.38960300 & 0.56596900 & 0.41967200 \\ \mathrm{H} & -6.02529900 & 0.36844200 & -0.45350400 \\ \mathrm{O} & -0.53538500 & -0.03216900 & -0.29095400 \\ \mathrm{C} & 0.65991600 & 0.45066300 & 0.07262600 \\ \mathrm{O} & 0.84589000 & 1.48127600 & 0.68100800 \\ \mathrm{~N} & 1.63495200 & -0.42194700 & -0.35835600 \\ \mathrm{H} & 1.28657500 & -1.24012000 & -0.83732900 \\ \mathrm{C} & 3.01459300 & -0.30970000 & -0.19798900 \\ \mathrm{C} & 3.80378600 & -1.34559900 & -0.72429700 \\ \mathrm{C} & 3.62871900 & 0.77246800 & 0.45118000 \\ \mathrm{C} & 5.18136100 & -1.31190400 & -0.61096800 \\ \mathrm{H} & 3.32526600 & -2.18188600 & -1.22518500 \\ \mathrm{C} & 5.01089500 & 0.80452700 & 0.56335000 \\ \mathrm{H} & 3.02282800 & 1.56854200 & 0.85795300 \\ \mathrm{C} & 5.76921400 & -0.23101600 & 0.03456900 \\ \mathrm{H} & 5.80525900 & -2.10147300 & -1.01020400 \\ \mathrm{H} & 5.51122800 & 1.62714100 & 1.05896000 \\ \mathrm{~N} & 7.22602500 & -0.18622400 & 0.15859800 \\ \mathrm{O} & 7.71587600 & 0.77341500 & 0.72662900 \\ \mathrm{O} & 7.86089200 & -1.11260300 & -0.31478100 \\ \mathrm{H} & -5.93643400 & 0.21108500 & 1.30129300 \\ \mathrm{H} & -2.79706000 & -1.81377500 & 0.01691000 \\ \mathrm{~N} & -4.61898000 & -2.10080200 & 0.13768600 \\ \mathrm{O} & -3.38016400 & -2.66315800 & -0.01822100 \\ \mathrm{C} & -5.41715200 & -2.36268800 & -1.05441900 \\ \mathrm{H} & -5.51837400 & -3.44057600 & -1.22410100 \\ \mathrm{H} & -6.40710800 & -1.91697500 & -0.93208200 \\ \mathrm{H} & -4.90784700 & -1.90597200 & -1.90426600 \\ \mathrm{C} & -5.21534100 & -2.59001600 & 1.37553900 \\ \mathrm{H} & -6.20194700 & -2.13879000 & 1.50480600 \\ \mathrm{H} & -5.31082500 & -3.68152000 & 1.35510500\end{array}$




\begin{tabular}{|c|c|c|c|}
\hline H & -4.56313600 & -2.29623800 & 2.19895800 \\
\hline \multicolumn{4}{|l|}{10} \\
\hline \multirow{2}{*}{\multicolumn{4}{|c|}{$\begin{array}{l}\text { Lowest three frequencies }\left(\mathrm{cm}^{-1}\right): 80.24,118.25,176.11 \\
\mathrm{E}(\mathrm{RM} 062 \mathrm{X})=-510.431597287\end{array}$}} \\
\hline & & & \\
\hline $\mathrm{C}$ & -1.67436000 & 1.18196600 & -0.40845200 \\
\hline $\mathrm{C}$ & -0.32963800 & 1.58007400 & 0.26692400 \\
\hline $\mathrm{C}$ & -0.76385600 & -1.45652300 & -0.06369900 \\
\hline $\mathrm{C}$ & 0.97268800 & 1.16247200 & -0.44240900 \\
\hline $\mathrm{C}$ & 0.40989900 & -1.17745400 & -0.07532500 \\
\hline $\mathrm{C}$ & 1.50492800 & -0.20197000 & -0.01613700 \\
\hline $\mathrm{H}$ & -1.48927800 & 0.85541900 & -1.43969700 \\
\hline $\mathrm{H}$ & -0.29796000 & 1.20361600 & 1.29604200 \\
\hline $\mathrm{H}$ & 0.83584900 & 1.13304100 & -1.52780100 \\
\hline $\mathrm{H}$ & -2.28308900 & 2.08742900 & -0.48968400 \\
\hline $\mathrm{H}$ & -0.30818000 & 2.67019500 & 0.35093800 \\
\hline $\mathrm{H}$ & $1.7^{\prime}$ & 1.87555500 & -0.22891000 \\
\hline $\mathrm{C}$ & -2.53922700 & 0.12193600 & 0.30852200 \\
\hline $\mathrm{H}$ & -3.59733800 & 0.30229800 & 0.09245900 \\
\hline $\mathrm{H}$ & -2.41556400 & 0.22915700 & 1.39161500 \\
\hline $\mathrm{C}$ & -2.21744000 & -1.34098700 & -0.08372900 \\
\hline $\mathrm{H}$ & -2.58554300 & -1.55164200 & -1.09394800 \\
\hline $\mathrm{H}$ & -2.70466700 & -2.04469400 & 0.59673400 \\
\hline $\mathrm{F}$ & 2.56186500 & -0.54323100 & -0.79918400 \\
\hline $\mathrm{F}$ & 1.98169000 & -0.12093100 & 1.25897000 \\
\hline
\end{tabular}

\section{0-TS}

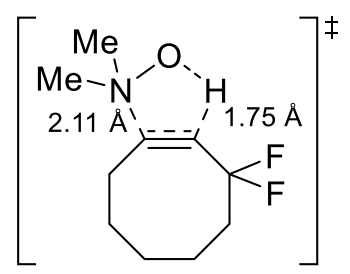

Lowest three frequencies $\left(\mathrm{cm}^{-1}\right)$ : $-118.45,41.71,43.87$ $\mathrm{E}(\mathrm{RM} 062 \mathrm{X})=-720.735442633$

$\begin{array}{lrrr}\mathrm{C} & 1.78908300 & 2.06449700 & -0.55671000 \\ \mathrm{C} & 2.80860800 & 0.92348400 & -0.35069600 \\ \mathrm{C} & -0.50780500 & 0.35230800 & 0.19602900 \\ \mathrm{C} & 2.43944300 & -0.48862700 & -0.81765600 \\ \mathrm{C} & 0.11225500 & -0.71562400 & 0.08518500 \\ \mathrm{C} & 1.48323600 & -1.24875200 & 0.08740400 \\ \mathrm{H} & 1.21974200 & 1.88579300 & -1.47867600 \\ \mathrm{H} & 3.10090400 & 0.87636100 & 0.70444100 \\ \mathrm{H} & 2.00889400 & -0.47065700 & -1.82418900 \\ \mathrm{H} & 2.36682200 & 2.97628200 & -0.73679600 \\ \mathrm{H} & 3.71590500 & 1.19352500 & -0.90078000 \\ \mathrm{H} & 3.34797400 & -1.09898300 & -0.86117500 \\ \mathrm{C} & 0.81552500 & 2.35434000 & 0.60613300 \\ \mathrm{H} & 0.73506000 & 3.43529200 & 0.76082500\end{array}$




$\begin{array}{lrrc}\mathrm{H} & 1.21375400 & 1.93061600 & 1.53481800 \\ \mathrm{C} & -0.60349100 & 1.80550400 & 0.38972900 \\ \mathrm{H} & -1.05521300 & 2.27659000 & -0.49268600 \\ \mathrm{H} & -1.24088300 & 2.04720600 & 1.24793800 \\ \mathrm{~F} & 1.50294900 & -2.56302200 & -0.29587800 \\ \mathrm{~F} & 1.97960900 & -1.23328700 & 1.36473800 \\ \mathrm{H} & -1.35352700 & -1.62930600 & -0.20414600 \\ \mathrm{~N} & -2.52977300 & -0.19823900 & -0.10633400 \\ \mathrm{C} & -3.09078800 & 0.39299700 & -1.31369900 \\ \mathrm{H} & -4.05539200 & -0.06475100 & -1.56362100 \\ \mathrm{H} & -3.22560600 & 1.46665000 & -1.16027000 \\ \mathrm{H} & -2.38702600 & 0.22259600 & -2.13003100 \\ \mathrm{C} & -3.33511700 & 0.00951500 & 1.09001700 \\ \mathrm{H} & -3.46900600 & 1.08199900 & 1.25228800 \\ \mathrm{H} & -4.31576800 & -0.47100900 & 0.99103200 \\ \mathrm{H} & -2.80052700 & -0.42733400 & 1.93478700 \\ \mathrm{O} & -2.36330300 & -1.54678400 & -0.30544600\end{array}$

\section{S24}

Lowest three frequencies $\left(\mathrm{cm}^{-1}\right): 84.47,121.18,175.30$ $\mathrm{E}(\mathrm{RM} 062 \mathrm{X})=-426.4666974$

$\begin{array}{lrrr}\mathrm{C} & -1.73300600 & 1.15677900 & -0.40840600 \\ \mathrm{C} & -0.39525900 & 1.58806600 & 0.26177300 \\ \mathrm{C} & -0.77146900 & -1.46130700 & -0.02376100 \\ \mathrm{C} & 0.91711500 & 1.18749600 & -0.44448100 \\ \mathrm{C} & 0.38566000 & -1.11568600 & -0.05106700 \\ \mathrm{C} & 1.52888700 & -0.18167200 & -0.04273400 \\ \mathrm{H} & -1.53921300 & 0.81449200 & -1.43285300 \\ \mathrm{H} & -0.37469900 & 1.22600200 & 1.29685900 \\ \mathrm{H} & 0.74712800 & 1.16148600 & -1.52885700 \\ \mathrm{H} & -2.35709700 & 2.05052600 & -0.50681200 \\ \mathrm{H} & -0.39754400 & 2.68012500 & 0.33422100 \\ \mathrm{H} & 1.69150100 & 1.94323700 & -0.26824300 \\ \mathrm{C} & -2.58026300 & 0.09325400 & 0.32395600 \\ \mathrm{H} & -3.64180700 & 0.25361900 & 0.10641900 \\ \mathrm{H} & -2.45875700 & 0.22077400 & 1.40552800 \\ \mathrm{C} & -2.22969500 & -1.36821100 & -0.04388700 \\ \mathrm{H} & -2.59858000 & -1.59961000 & -1.04984000 \\ \mathrm{O} & 2.56155000 & -0.53459400 & -0.94648900 \\ \mathrm{H} & -2.71061100 & -2.06817000 & 0.64567400 \\ \mathrm{H} & 2.15405200 & -0.70694600 & -1.80337500 \\ \mathrm{C} & 2.19440900 & -0.12560300 & 1.32862000 \\ \mathrm{H} & 2.57399000 & -1.11348200 & 1.59485200 \\ \mathrm{H} & 3.03266300 & 0.57451000 & 1.28390600 \\ \mathrm{H} & 1.48830100 & 0.20148200 & 2.09435500\end{array}$

\section{S24-TS}




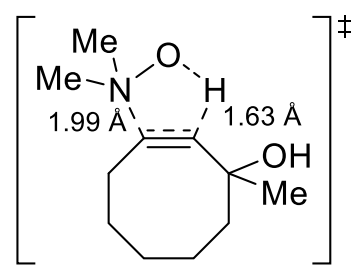

Lowest three frequencies $\left(\mathrm{cm}^{-1}\right):-187.48,46.07,52.59$ $\mathrm{E}(\mathrm{RM} 062 \mathrm{X})=-636.7643389$

$\begin{array}{lrrr}\mathrm{C} & -1.68999200 & 2.12745800 & 0.56967000 \\ \mathrm{C} & -2.76121700 & 1.02803400 & 0.43710300 \\ \mathrm{C} & 0.57426400 & 0.33503100 & -0.19723100 \\ \mathrm{C} & -2.40082400 & -0.40642100 & 0.84145200 \\ \mathrm{C} & -0.09183900 & -0.71852300 & -0.05941600 \\ \mathrm{C} & -1.48388400 & -1.24394500 & -0.07345400 \\ \mathrm{H} & -1.08494100 & 1.93637800 & 1.46608800 \\ \mathrm{H} & -3.16652600 & 1.03940200 & -0.58272300 \\ \mathrm{H} & -1.94869800 & -0.39256900 & 1.84298000 \\ \mathrm{H} & -2.21837700 & 3.06736000 & 0.75927000 \\ \mathrm{H} & -3.59875000 & 1.31587700 & 1.08282000 \\ \mathrm{H} & -3.32944800 & -0.98282100 & 0.92764200 \\ \mathrm{C} & -0.76060900 & 2.34614900 & -0.64197000 \\ \mathrm{H} & -0.67171200 & 3.41719000 & -0.85285800 \\ \mathrm{H} & -1.20070000 & 1.89040700 & -1.53654500 \\ \mathrm{C} & 0.65516700 & 1.78760700 & -0.45027300 \\ \mathrm{H} & 1.13354100 & 2.30102300 & 0.39472000 \\ \mathrm{O} & -1.53729100 & -2.59462700 & 0.39416400 \\ \mathrm{H} & 1.26309400 & 2.00192000 & -1.33781200 \\ \mathrm{H} & -0.99826600 & -2.63590400 & 1.19205000 \\ \mathrm{H} & 1.24989700 & -1.61226700 & 0.19765200 \\ \mathrm{~N} & 2.46289100 & -0.22552300 & 0.10719100 \\ \mathrm{C} & 3.00775000 & 0.38523900 & 1.31591200 \\ \mathrm{H} & 3.96444100 & -0.07785400 & 1.58021200 \\ \mathrm{H} & 3.15075200 & 1.45568700 & 1.15187600 \\ \mathrm{H} & 2.28880300 & 0.22519700 & 2.12049300 \\ \mathrm{C} & 3.27620300 & -0.01588000 & -1.08692000 \\ \mathrm{H} & 3.40465800 & 1.05565200 & -1.25646700 \\ \mathrm{H} & 4.25628400 & -0.49082000 & -0.97042500 \\ \mathrm{H} & 2.74939000 & -0.46587000 & -1.92912400 \\ \mathrm{O} & 2.29614000 & -1.56352200 & 0.31329600 \\ \mathrm{C} & -2.01316100 & -1.31791900 & -1.50474700 \\ \mathrm{H} & -1.37752400 & -1.98744400 & -2.08708300 \\ \mathrm{H} & -3.02967900 & -1.72302000 & -1.49520100 \\ \mathrm{H} & -2.01841000 & -0.33464900 & -1.97834500\end{array}$

\section{S25}

Lowest three frequencies $\left(\mathrm{cm}^{-1}\right): 29.25,209.73,209.75$

$\mathrm{E}(\mathrm{RM} 062 \mathrm{X})=-155.9439042$

$\begin{array}{llll}\mathrm{C} & 0.00000000 & 0.00000000 & 0.60299000\end{array}$

$\begin{array}{lllll}\mathrm{C} & 0.00000000 & 0.00000000 & -0.60299000\end{array}$ 


$\begin{array}{lrcc}\mathrm{C} & 0.00000000 & 0.00000000 & 2.06570800 \\ \mathrm{H} & -0.51002300 & 0.88438900 & 2.45675800 \\ \mathrm{H} & -0.51089200 & -0.88388800 & 2.45675800 \\ \mathrm{H} & 1.02091500 & -0.00050100 & 2.45675800 \\ \mathrm{C} & 0.00000000 & 0.00000000 & -2.06570800 \\ \mathrm{H} & -1.02091500 & -0.00050100 & -2.45675800 \\ \mathrm{H} & 0.51002300 & 0.88438900 & -2.45675800 \\ \mathrm{H} & 0.51089200 & -0.88388800 & -2.45675800\end{array}$

\section{S25-TS}

Lowest three frequencies $\left(\mathrm{cm}^{-1}\right):-356.05,62.60,95.08$

$\mathrm{E}(\mathrm{RM} 062 \mathrm{X})=-366.217519$

$\begin{array}{lrrr}\mathrm{C} & 1.55364700 & -0.29356100 & -0.00038600 \\ \mathrm{C} & 0.70913100 & 0.63801200 & -0.00196200 \\ \mathrm{C} & 3.01745300 & -0.55067300 & 0.00020500 \\ \mathrm{~N} & -1.02763500 & -0.30894300 & 0.00011900 \\ \mathrm{C} & -1.77383500 & -0.03174000 & 1.22452700 \\ \mathrm{H} & -2.10624700 & 1.00897600 & 1.22606200 \\ \mathrm{H} & -2.64230900 & -0.69483200 & 1.29783000 \\ \mathrm{C} & -1.77405100 & -0.03744700 & -1.22548900 \\ \mathrm{H} & -2.10383100 & 1.00408500 & -1.23355100 \\ \mathrm{H} & -2.64433000 & -0.69869700 & -1.29412600 \\ \mathrm{O} & -0.60849600 & -1.60041300 & 0.00312600 \\ \mathrm{H} & 0.45876100 & -1.40079900 & 0.00222100 \\ \mathrm{H} & -1.10531100 & -0.22433000 & -2.06630500 \\ \mathrm{H} & -1.10413800 & -0.21152800 & 2.06617700 \\ \mathrm{H} & 3.30073400 & -1.13740500 & 0.87915300 \\ \mathrm{H} & 3.61902700 & 0.36485400 & -0.00495500 \\ \mathrm{H} & 3.30037700 & -1.14716800 & -0.87224100 \\ \mathrm{C} & 0.32711400 & 2.06639100 & -0.00094900 \\ \mathrm{H} & 1.23858300 & 2.67128300 & -0.00490500 \\ \mathrm{H} & -0.25716800 & 2.34274900 & -0.88438300 \\ \mathrm{H} & -0.24949300 & 2.34282600 & 0.88750400\end{array}$

\section{$\mathrm{NMe}_{2} \mathrm{OH}$}

Lowest three frequencies $\left(\mathrm{cm}^{-1}\right): 251.20,290.61,315.61$ $\mathrm{E}(\mathrm{RM} 062 \mathrm{X})=-210.302452064$

$\begin{array}{lrrc}\mathrm{N} & 0.00000000 & 0.02490000 & -0.41697400 \\ \mathrm{C} & -1.19858000 & -0.64238700 & 0.06738400 \\ \mathrm{H} & -1.22931400 & -1.65128600 & -0.35177800 \\ \mathrm{H} & -1.22122900 & -0.70365200 & 1.16601500 \\ \mathrm{C} & 1.19858300 & -0.64238200 & 0.06738400 \\ \mathrm{H} & 1.22932400 & -1.65127900 & -0.35178200 \\ \mathrm{H} & 1.22123100 & -0.70365100 & 1.16601500 \\ \mathrm{O} & -0.00000300 & 1.31390800 & 0.19839700 \\ \mathrm{H} & -0.00000300 & 1.91418700 & -0.55581600 \\ \mathrm{H} & 2.07548200 & -0.09062800 & -0.27480500 \\ \mathrm{H} & -2.07548200 & -0.09063900 & -0.27480900\end{array}$




\section{References}

(1) Hagendorn, T.; Bräse, S., A Route to Cyclooct-2-ynol and Its Functionalization by Mitsunobu Chemistry. Eur. J. Org. Chem. 2014, 2014, 1280.

(2) Fairbanks, B. D.; Sims, E. A.; Anseth, K. S.; Bowman, C. N., Reaction Rates and Mechanisms for Radical, Photoinitated Addition of Thiols to Alkynes, and Implications for Thiol-Yne Photopolymerizations and Click Reactions. Macromolecules 2010, 43, 4113.

(3) Madea, D.; Slanina, T.; Klán, P., A 'photorelease, catch and photorelease' strategy for bioconjugation utilizing a p-hydroxyphenacyl group. Chem. Commun. (Cambridge, U. K.) 2016, 52 , 12901.

(4) Heller, K.; Ochtrop, P.; Albers, M. F.; Zauner, F. B.; Itzen, A.; Hedberg, C., Covalent Protein Labeling by Enzymatic Phosphocholination. Angew. Chem., Int. Ed. 2015, 54, 10327.

(5) Plass, T.; Milles, S.; Koehler, C.; Schultz, C.; Lemke, E. A., Genetically Encoded CopperFree Click Chemistry. Angew. Chem., Int. Ed. 2011, 50, 3878.

(6) Fischer, A. H.; Jacobson, K. A.; Rose, J.; Zeller, R., Preparation of slides and coverslips for microscopy. CSH Protoc 2008, 2008, pdb.prot4988.

(7) Gaussian 16, Revision C.01, M. J. Frisch, G. W. Trucks, H. B. Schlegel, G. E. Scuseria, M. A. Robb, J. R. Cheeseman, G. Scalmani, V. Barone, G. A. Petersson, H. Nakatsuji, X. Li, M. Caricato, A. V. Marenich, J. Bloino, B. G. Janesko, R. Gomperts, B. Mennucci, H. P. Hratchian, J. V. Ortiz, A. F. Izmaylov, J. L. Sonnenberg, D. Williams-Young, F. Ding, F. Lipparini, F. Egidi, J. Goings, B. Peng, A. Petrone, T. Henderson, D. Ranasinghe, V. G. Zakrzewski, J. Gao, N. Rega, G. Zheng, W. Liang, M. Hada, M. Ehara, K. Toyota, R. Fukuda, J. Hasegawa, M. Ishida, T. Nakajima, Y. Honda, O. Kitao, H. Nakai, T. Vreven, K. Throssell, J. A. Montgomery, Jr., J. E. Peralta, F. Ogliaro, M. J. Bearpark, J. J. Heyd, E. N. Brothers, K. N. Kudin, V. N. Staroverov, T. A. Keith, R. Kobayashi, J. Normand, K. Raghavachari, A. P. Rendell, J. C. Burant, S. S. Iyengar, J. Tomasi, M. Cossi, J. M. Millam, M. Klene, C. Adamo, R. Cammi, J. W. Ochterski, R. L. Martin, K. Morokuma, O. Farkas, J. B. Foresman, and D. J. Fox, Gaussian, Inc., Wallingford CT, 2019.

(8) Zhao, Y.; Truhlar, D. G., The M06 suite of density functionals for main group thermochemistry, thermochemical kinetics, noncovalent interactions, excited states, and transition elements: two new functionals and systematic testing of four M06-class functionals and 12 other functionals. Theor. Chem. Acc. 2008, 120, 215.

(9) CYLview, 1.0b; Legault, C. Y., Université de Sherbrooke, 2009 (http://www.cylview.org). 


\section{NMR Spectra}




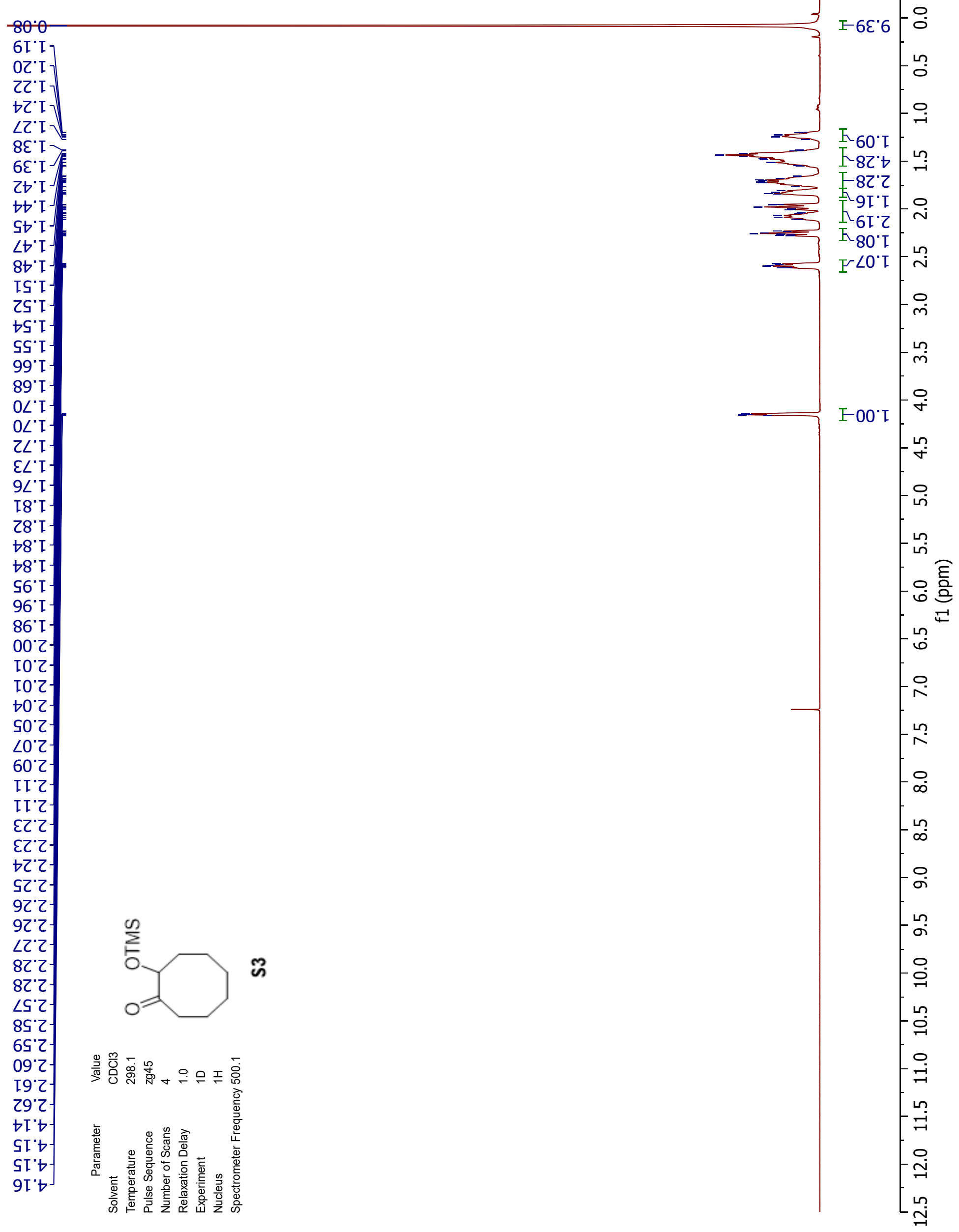


Bioorthogonal retro-Cope elimination reaction of N,N-dialkylhydroxylamines and strained alkynes Dahye Kang and Justin Kim*

$$
\text { 乙"0- }
$$

†'IZ$\varepsilon \cdot s Z-$ I.92 $9 \tau^{-}$ 乙'L乙

$\angle " t \varepsilon^{-}$

I.6ع-
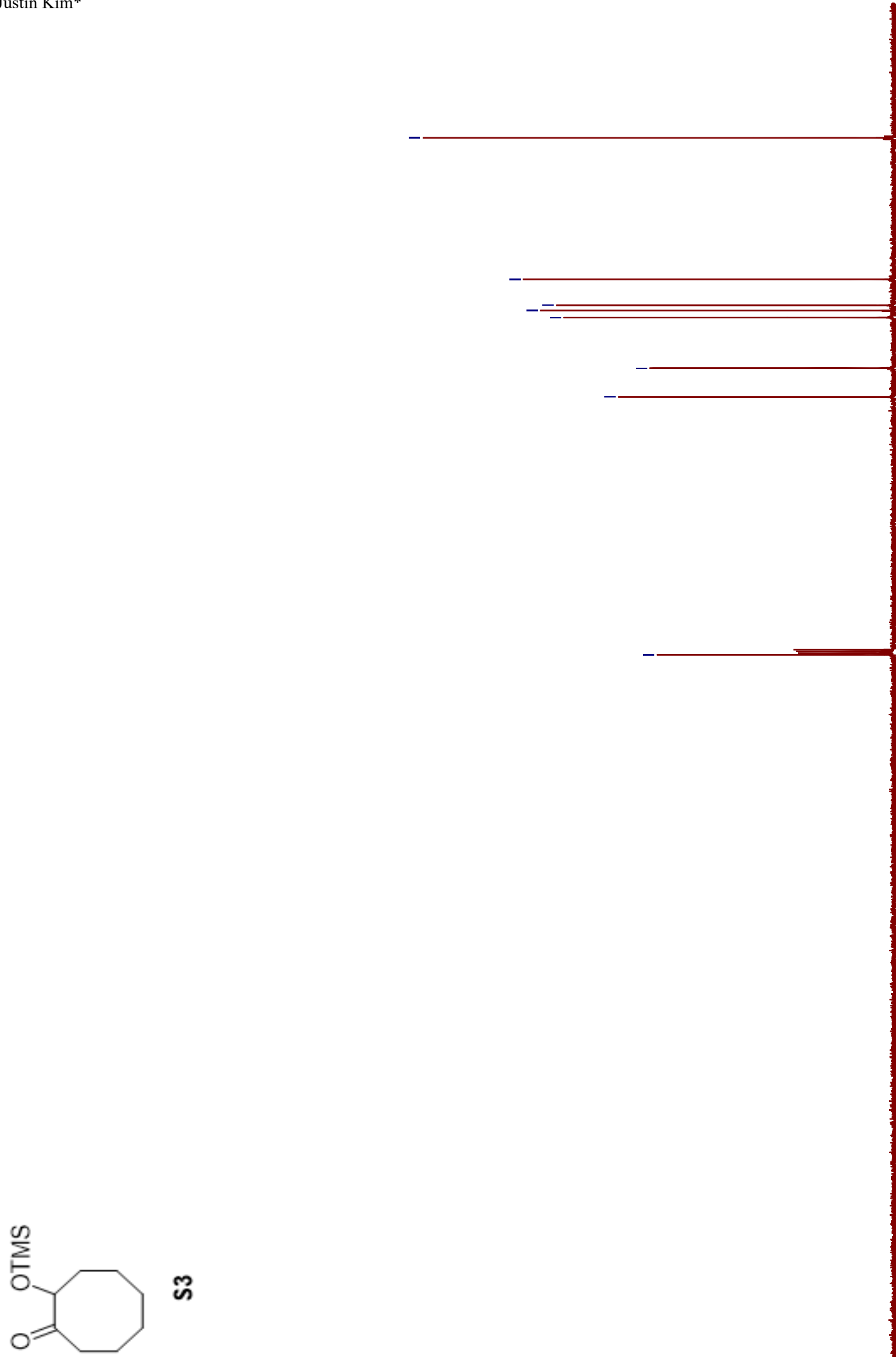

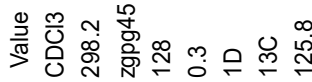


OS' $I$

OS' I -

9S. I -

$\angle S^{\prime} I$

$8 S^{\prime} \mathrm{I}$

6S' I

09. I

I9' I

$0 \angle$ '

$\mathrm{I} L \cdot \mathrm{I}$

$\mathrm{L} L \cdot \mathrm{T}$

$\varepsilon L$ ' I

$S L ' I$

$\angle L^{\prime} I$ -

$\angle L ' I-$

08 ' I -

I8. I-

90 '

$60^{\prime} \textrm{ }$

OI'乙-

$\angle Z^{\prime} 乙$

$\angle Z \cdot Z$

$6 \sigma^{\prime} 乙-$

乙๕'

เด

s9"

$99^{\circ} t$

$\angle 9^{\circ} \triangleright$

99'S

89's

69.

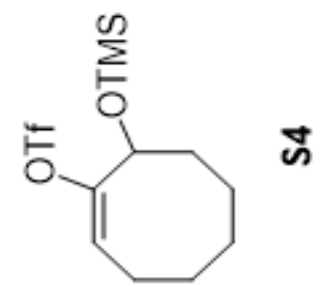

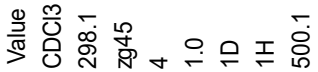
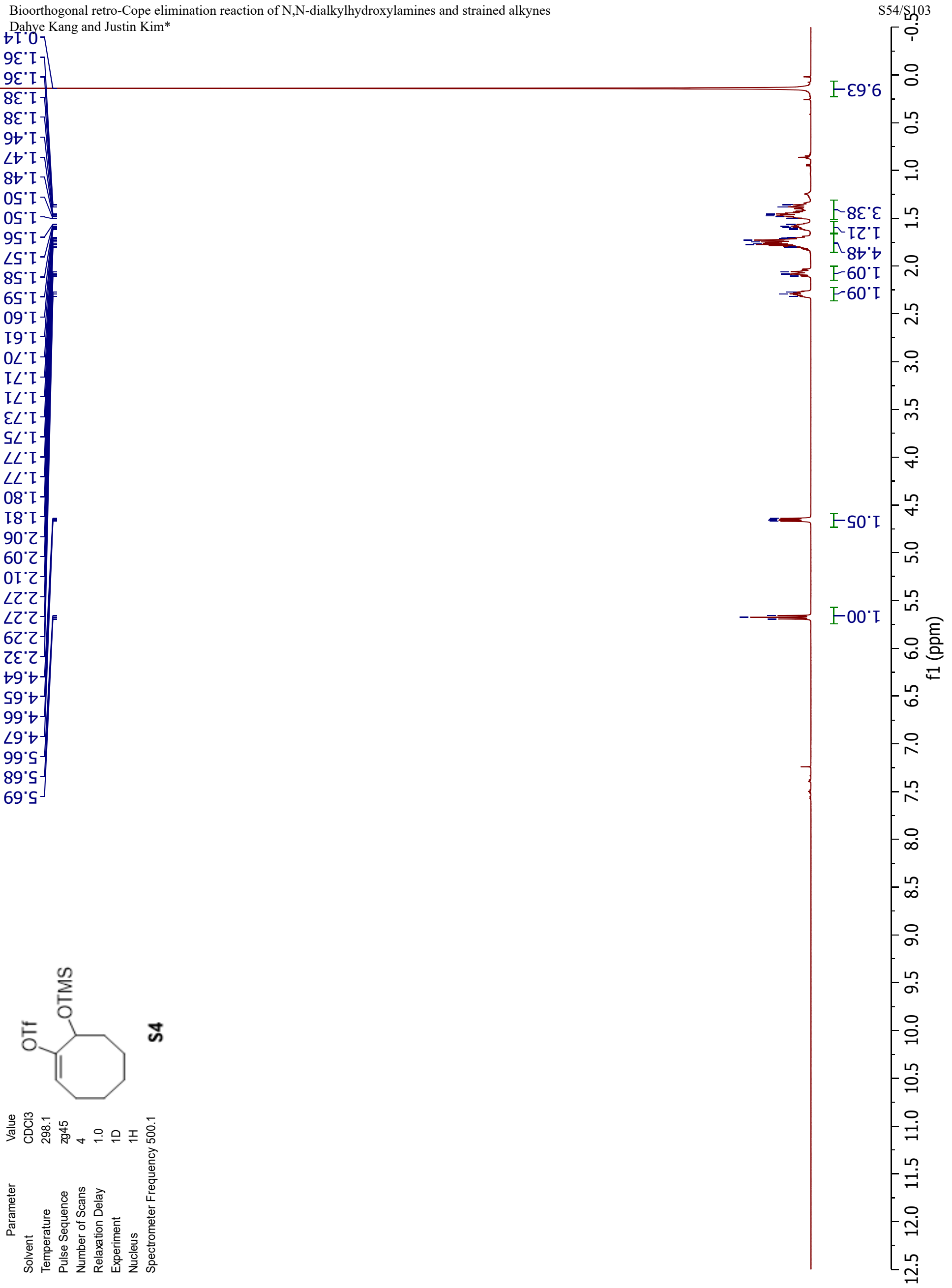
Bioorthogonal retro-Cope elimination reaction of N,N-dialkylhydroxylamines and strained alkynes Dahye Kang and Justin Kim*

I'0- -

9.ยน-

$8 . t 25$

$2.97 /$

$0 . \angle \varepsilon^{-}$

$\varepsilon: \angle 9-$

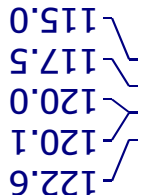

9.0SI -

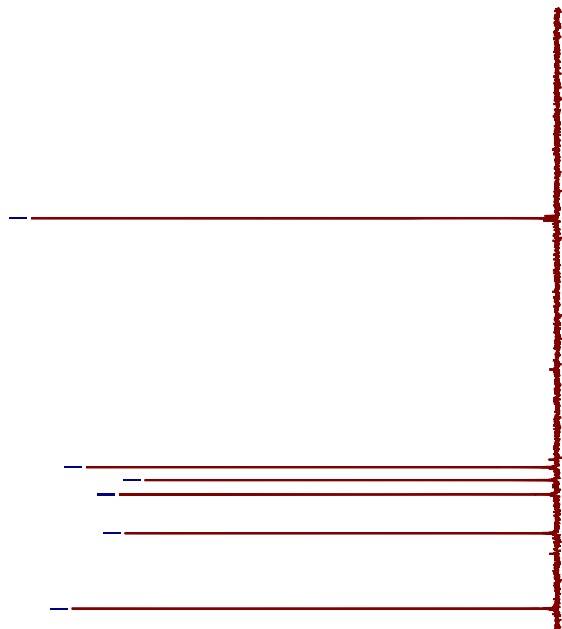

$-$

$-0$

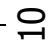

으

- 요

우

으

$-8$

R

$-\infty$

8

- 응 흘

윽

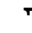

우

음

욱

윰

$-8$

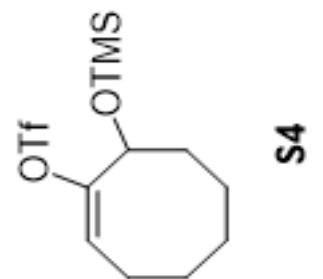

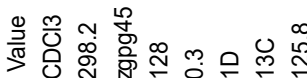

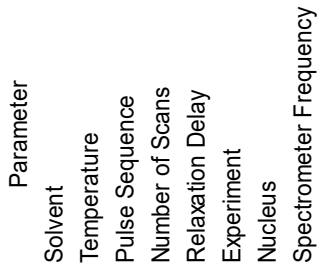


Bioorthogonal retro-Cope elimination reaction of N,N-dialkylhydroxylamines and strained alkynes Dahye Kang and Justin Kim*

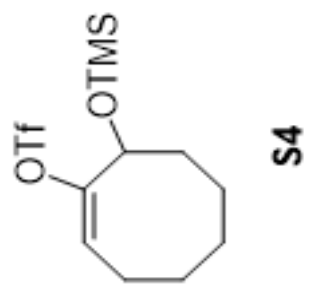




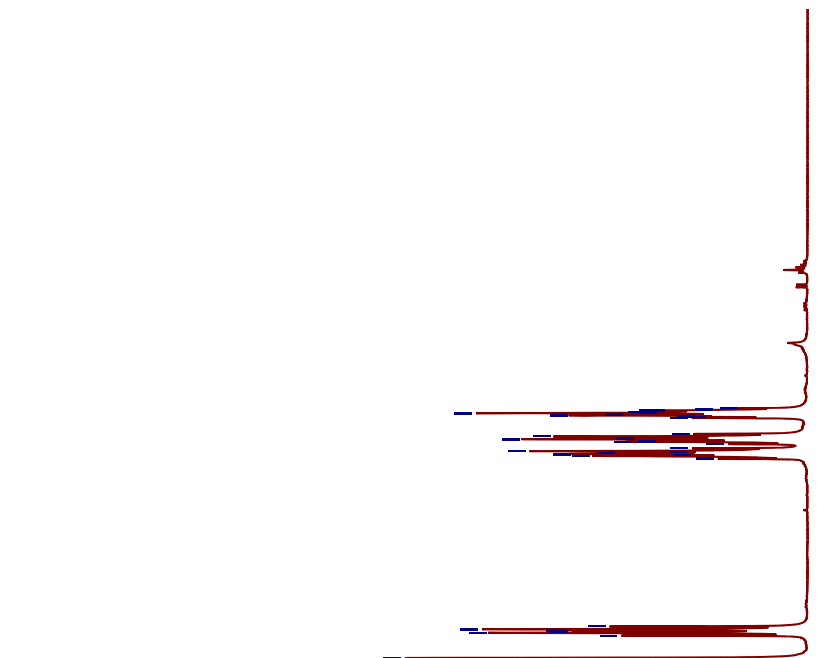

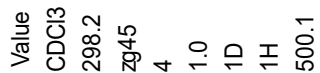


Bioorthogonal retro-Cope elimination reaction of N,N-dialkylhydroxylamines and strained alkynes
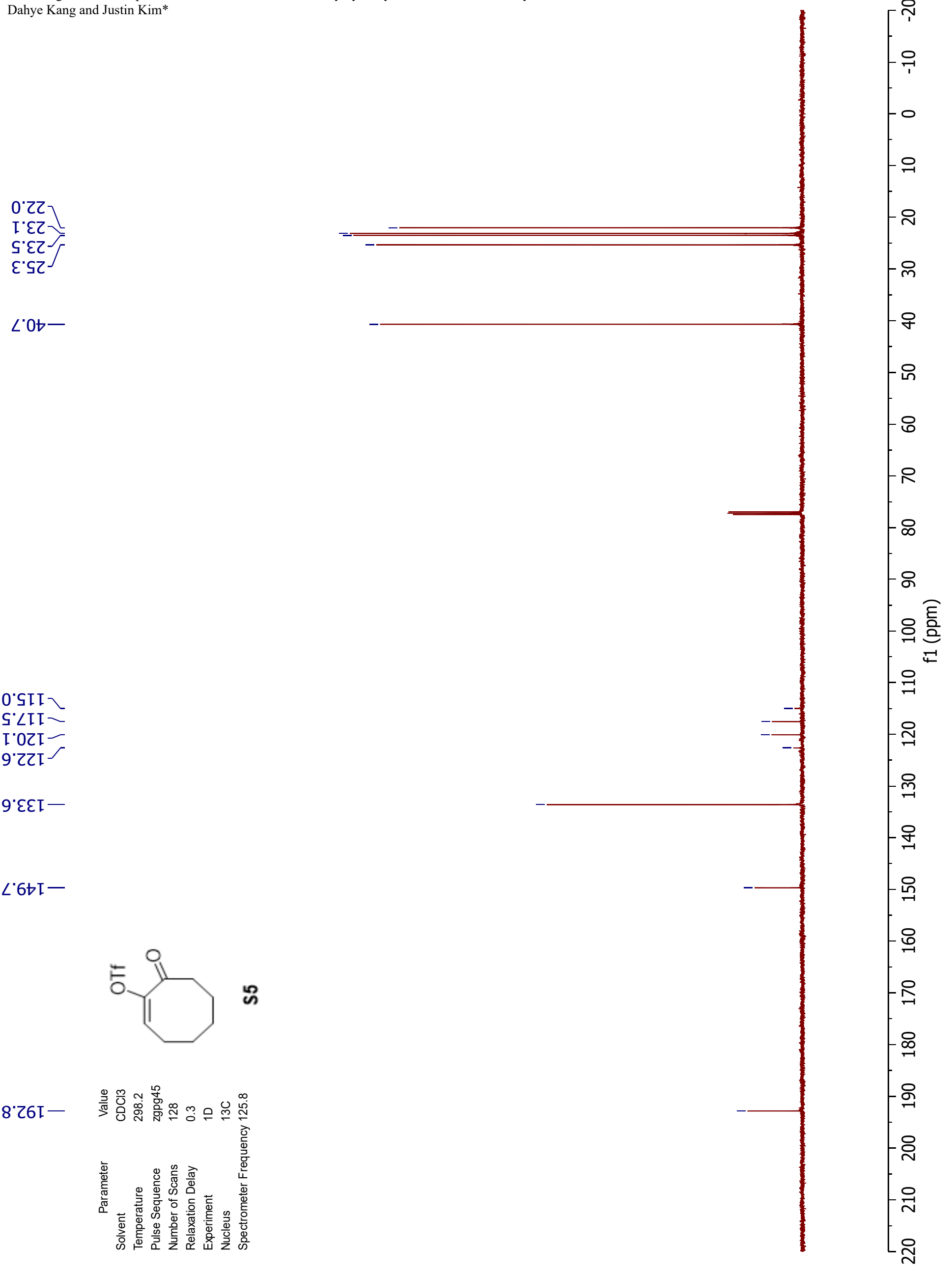

9 CZL

$9^{*} \varepsilon \varepsilon I-$

$\angle \cdot 6 t I-$ 
Bioorthogonal retro-Cope elimination reaction of N,N-dialkylhydroxylamines and strained alkynes Dahye Kang and Justin Kim*

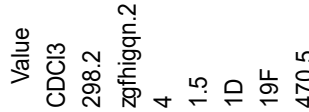


Bioorthogonal retro-Cope elimination reaction of N,N-dialkylhydroxylamines and strained alkynes 6sahve Kang and Justin Kim*

$6 S^{\prime} \mathrm{T}$

$09^{\circ} \mathrm{I}$

โร' $\mathrm{T}$

Z9. I -

Z9. I

$\varepsilon 9^{*} \mathrm{I}$

$\varepsilon 9^{\prime} \mathrm{T}$

เ9. $\mathrm{s} \cdot \mathrm{T}$

I0'Z]

$20 \cdot 2]$

$\varepsilon 0^{\circ} Z-$

$\varepsilon t^{\prime} Z$

$\varepsilon t^{\prime} z-$

t大'

st'

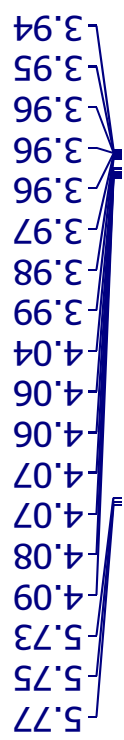

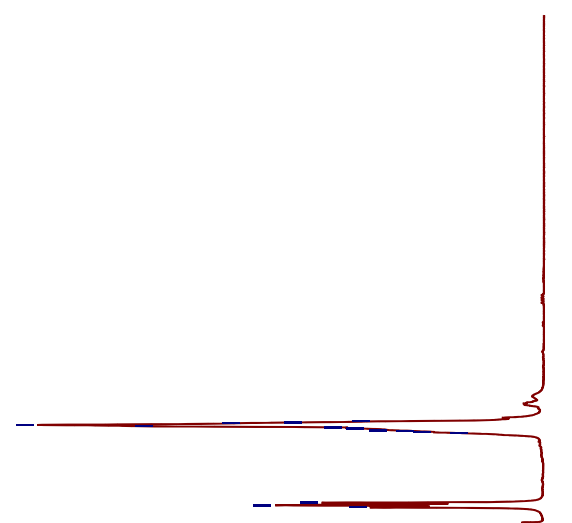

$\mathrm{S} 60 / \mathrm{Sn}^{03}$

$\AA_{6 I} \cdot 9$

$6 I .9$

F $00.2-\stackrel{\circ}{\sim}$ $\tau^{00} \mathrm{C}$

$-$

ก

m.

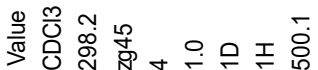
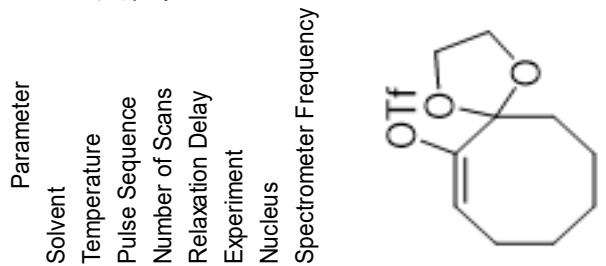

เก

웅

4

ซ்

$ㅇ$

신

눈

r

$\infty$

$\infty$

웅

กั

웅

음

웅

을

음

$\begin{array}{r}0 \\ -1 \\ \hline\end{array}$

근

$\stackrel{1}{\sim}$ 
Bioorthogonal retro-Cope elimination reaction of N,N-dialkylhydroxylamines and strained alkynes Dahye Kang and Justin Kim*

6. 12

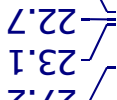

$\tau^{\prime}\left\llcorner\tau^{\top}\right.$

$\angle \cdot L \varepsilon-$

L’s9-

$\varepsilon^{\prime} \angle O T$

$6 \circ I I$

$\left.t^{\circ} \angle I T\right]$

6.6II

S'ZZI

0 'ยนโ

I'OSI -

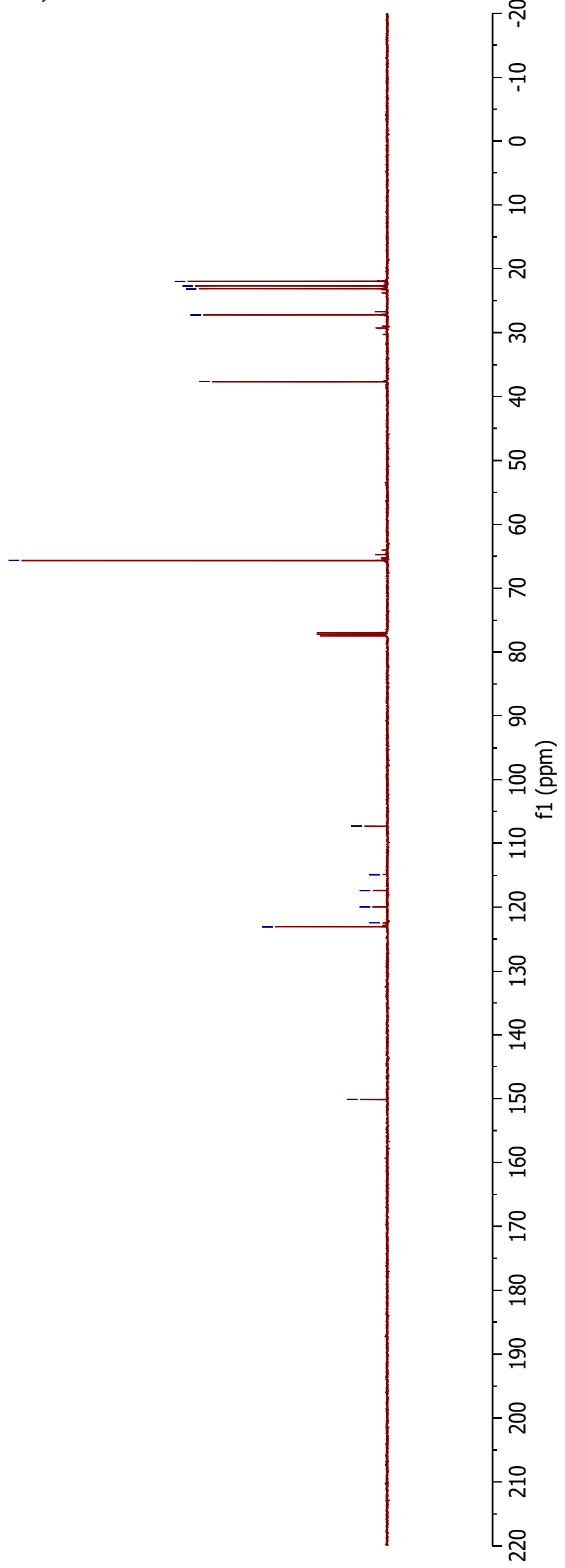

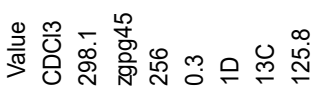
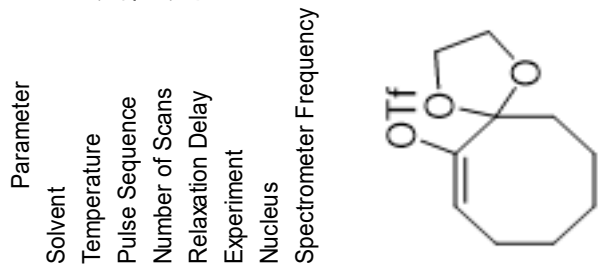

is 
Bioorthogonal retro-Cope elimination reaction of N,N-dialkylhydroxylamines and strained alkynes Dahye Kang and Justin Kim*

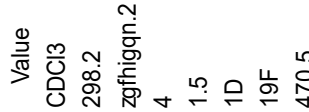
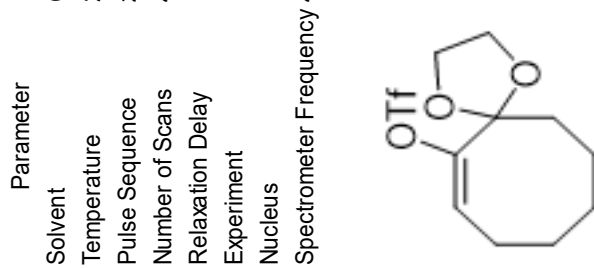
Bioorthogonal retro-Cope elimination reaction of N,N-dialkylhydroxylamines and strained alkynes Dahye Kang and Justin Kim*
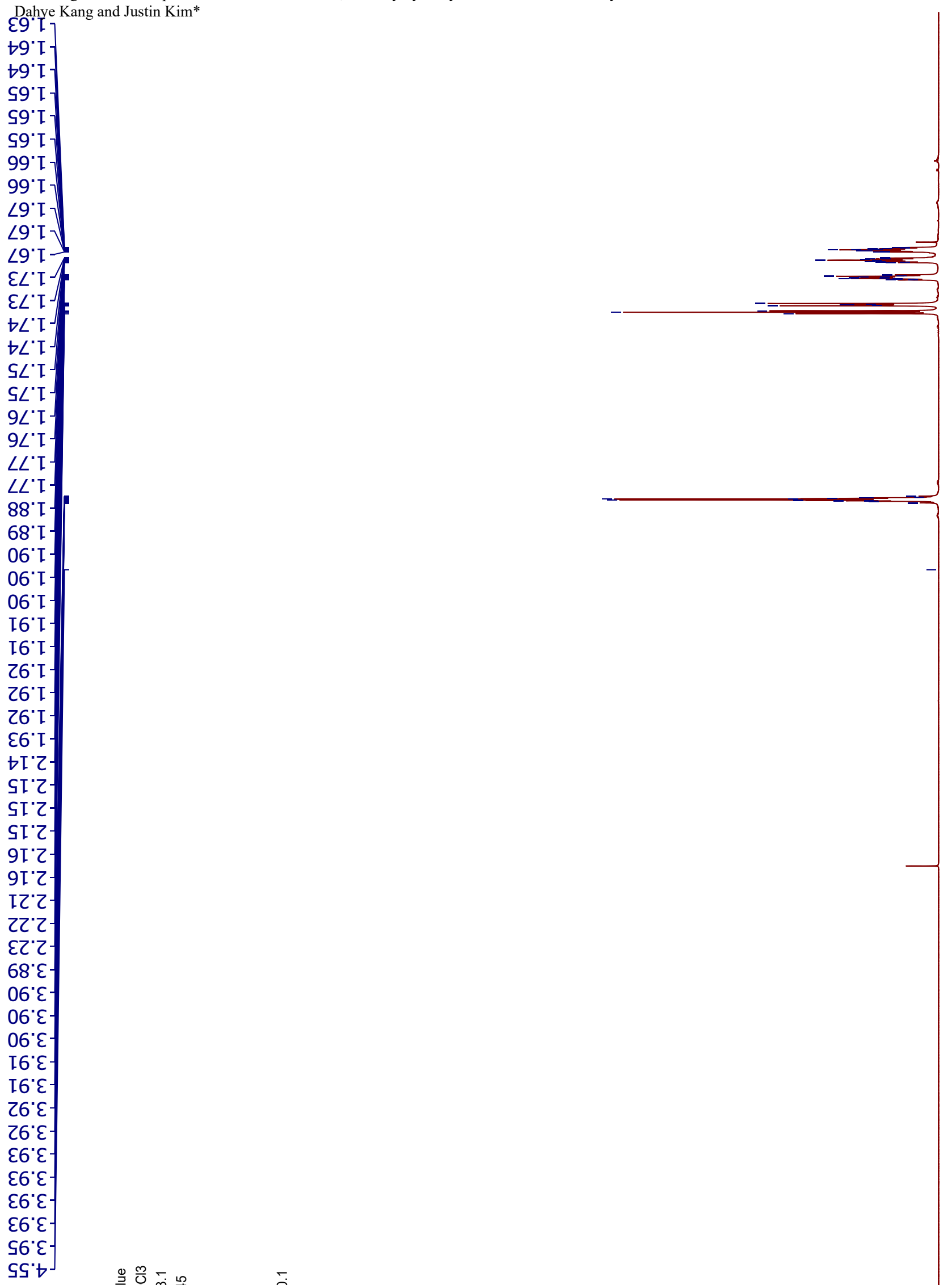

$\succ 9^{\circ} \mathrm{I}$

S9. I

S9' 1

99. I

99.

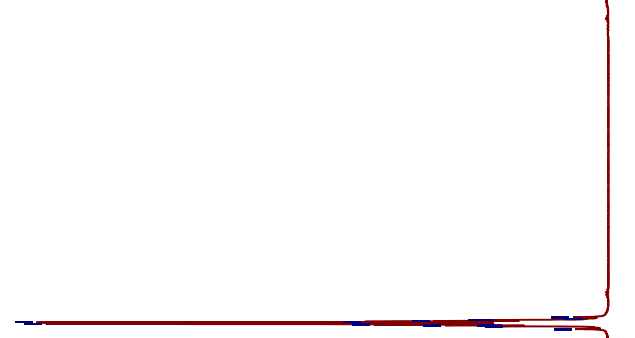

$=-600^{\circ} 2$

К $50 \cdot 2$ 每90'乙

lo0?

$\stackrel{\sim}{\sim}$

-

ํㅜㅁ

mก

Fโ6 ${ }^{\circ} \varepsilon$

-

$-2$

$\sigma$

-웅

- ᄂ?

- ํㅡㅇ

느

ขอ

$-0$

$-1$

는

잉

$\infty$

농

$-0$

$\sigma$

ตั

응

응

윽

눅
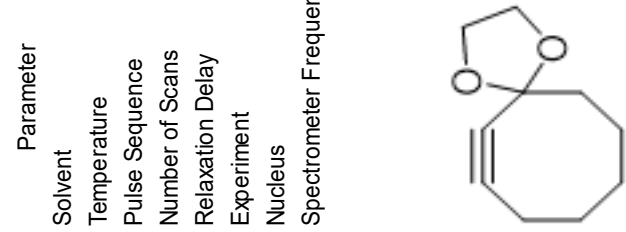
Bioorthogonal retro-Cope elimination reaction of N,N-dialkylhydroxylamines and strained alkynes Dahye Kang and Justin Kim*

$9^{\circ} 02^{-}$

$0 . \angle Z \backslash$

$8.62-$

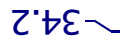

$b^{\prime} \angle b-$

$\angle ゙ \circ 9-$

$8.68-$

E'SOL

๖'LOI -

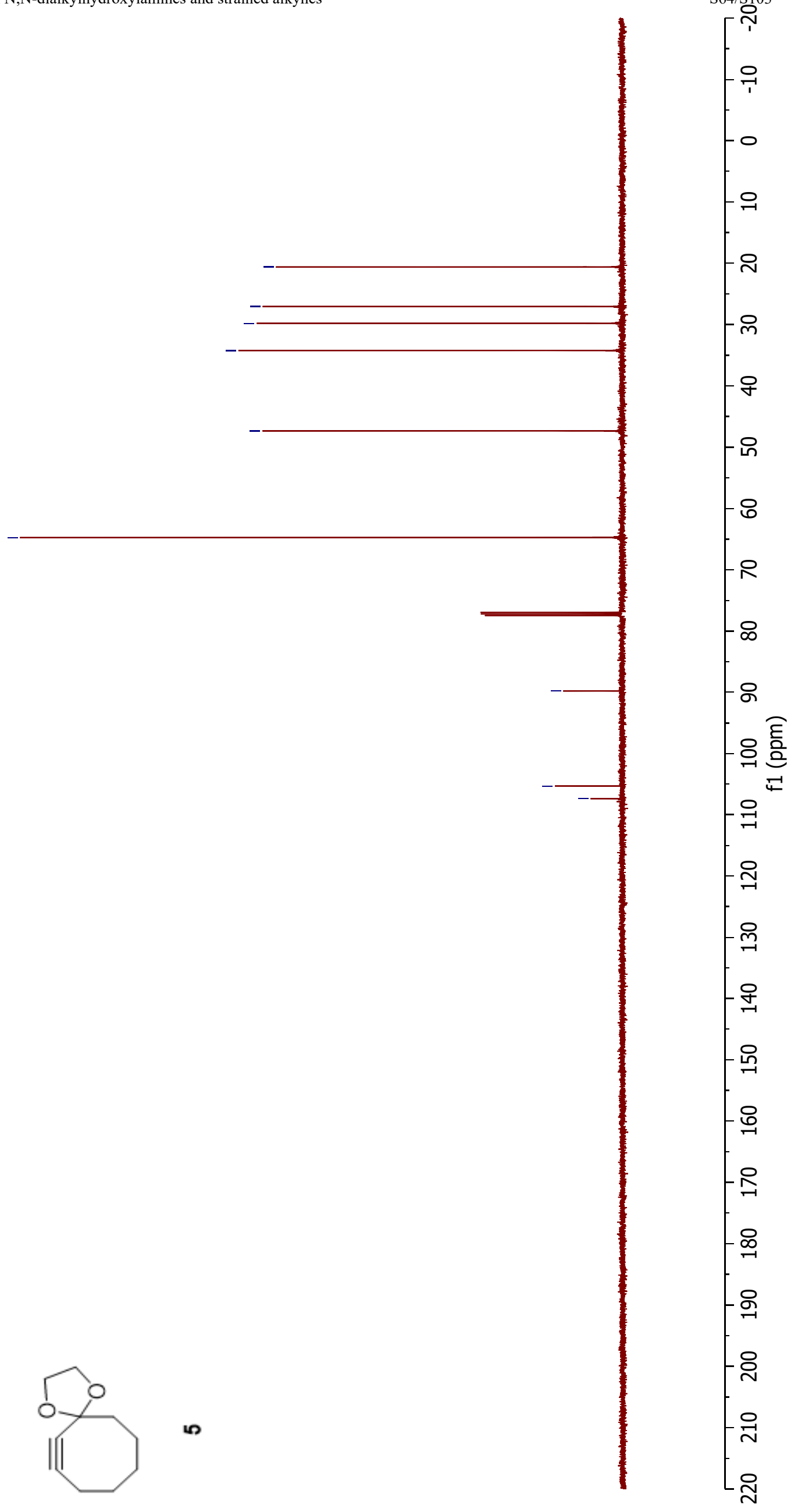

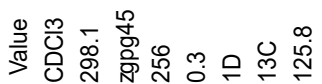

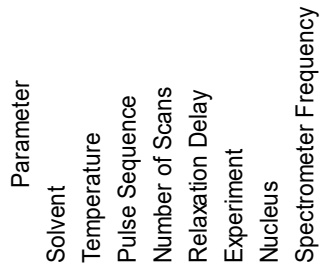




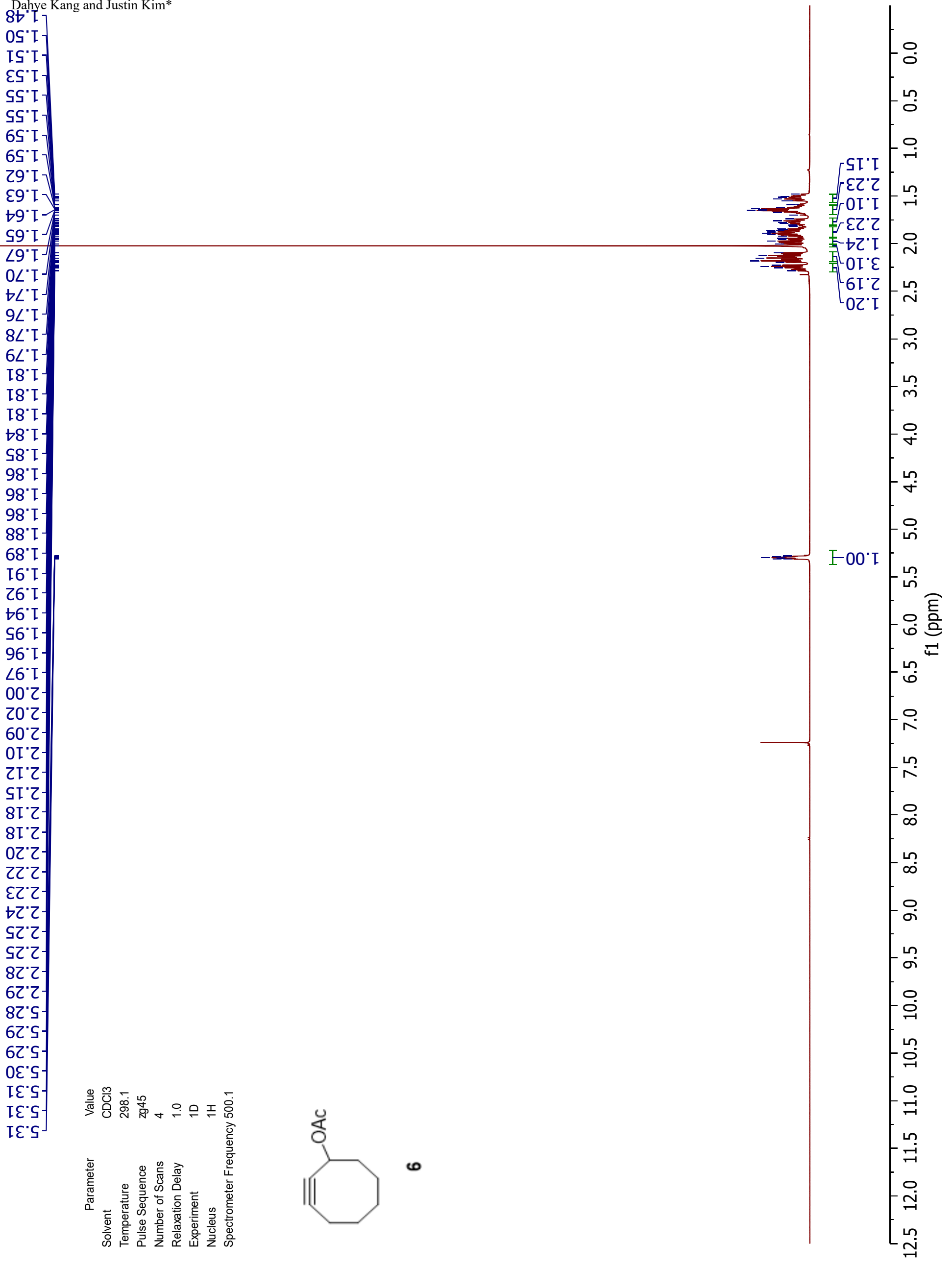




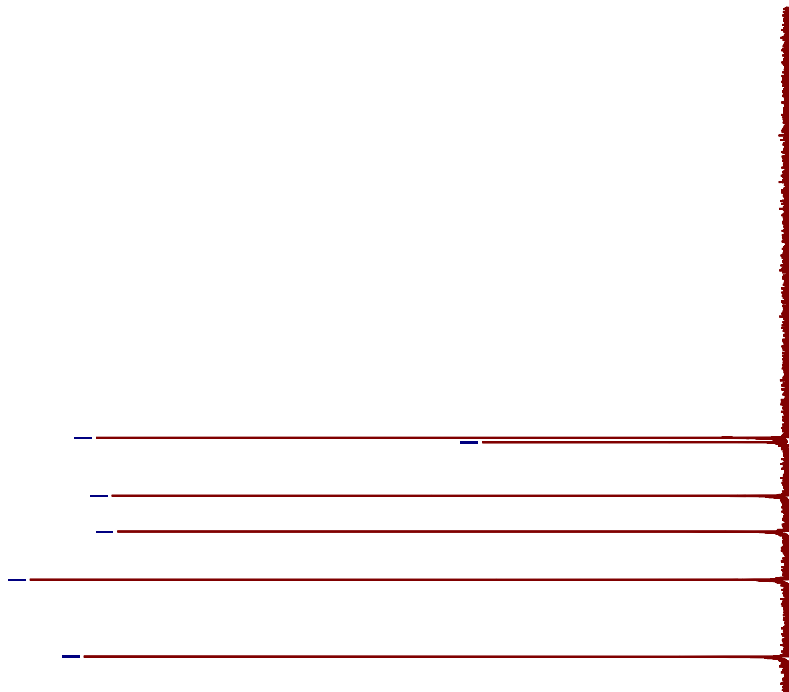

$\angle \cdot 99-$

$8 \cdot 06-$

0`ZOI -

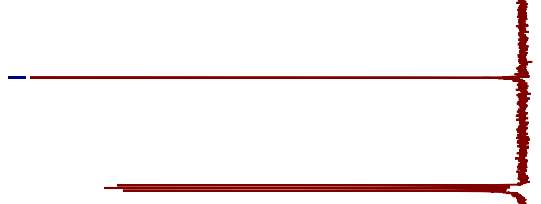

R

$\infty$

8

- 응 흘

윽

음

고

요

온

윰

$-8$

웅

옥

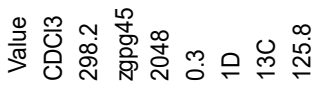
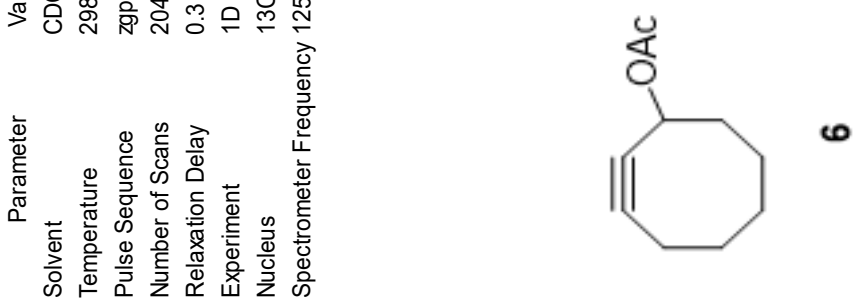

유

윽

ํำ 
$6 t^{\circ}$

OS

ZS'

$\downarrow S^{\prime}$

85

ZL'T]

$t L \cdot I$

$9 \angle$ ' I

68

06

$26^{\circ}$

90'

80 '

$60 \mathrm{c}$

9I' 2

$8 \mathrm{I} \cdot \mathrm{Z}$

IZ'Z

ZZ' 2

๑ै'

90 '

8 I'S $^{\prime}$

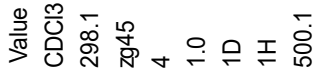
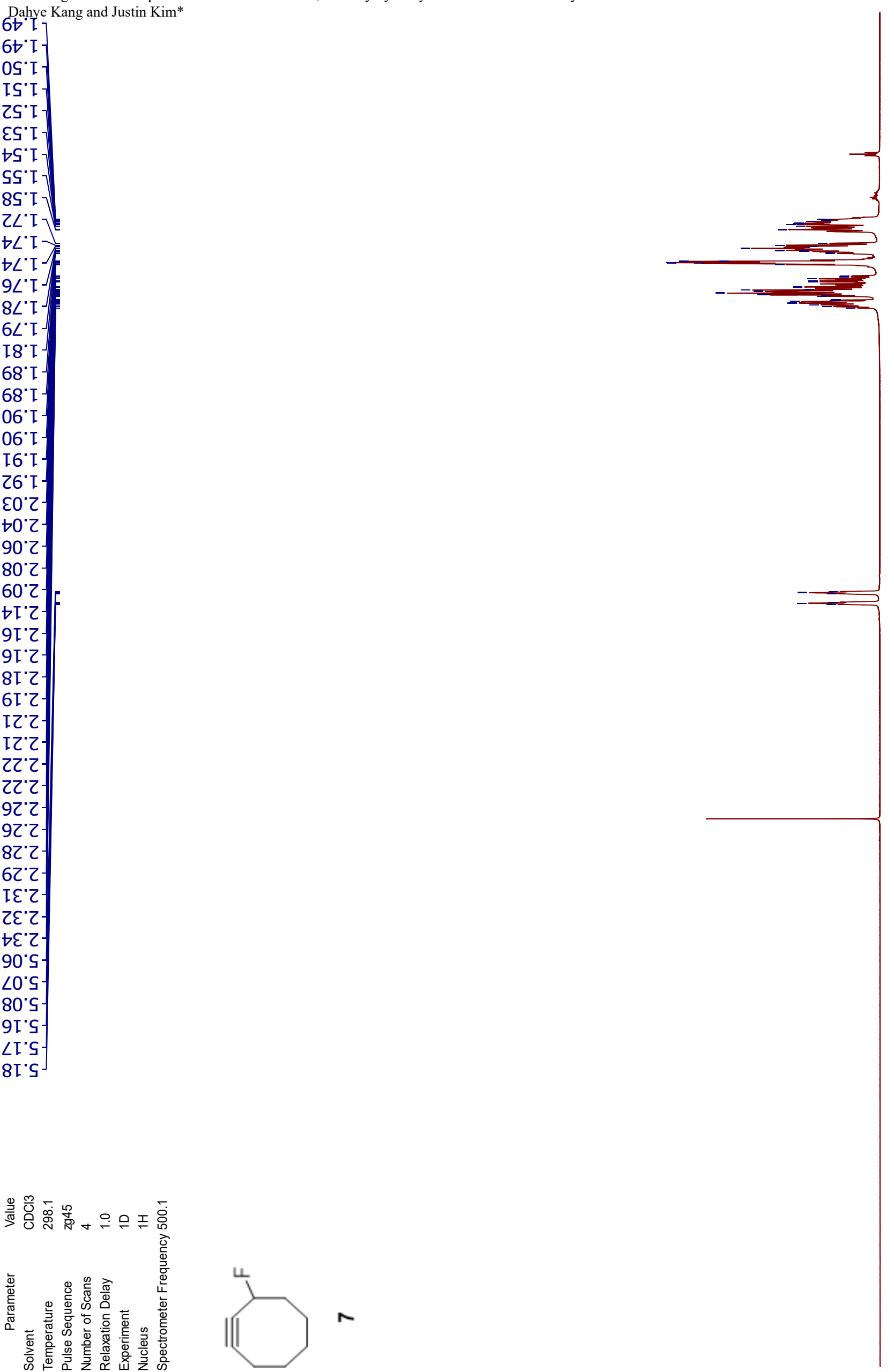

厂โE

五 $12 \cdot 2$

F 82"
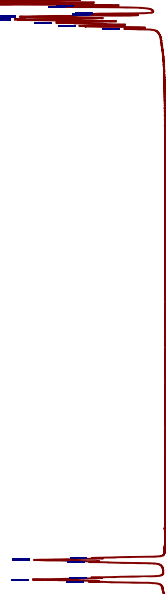


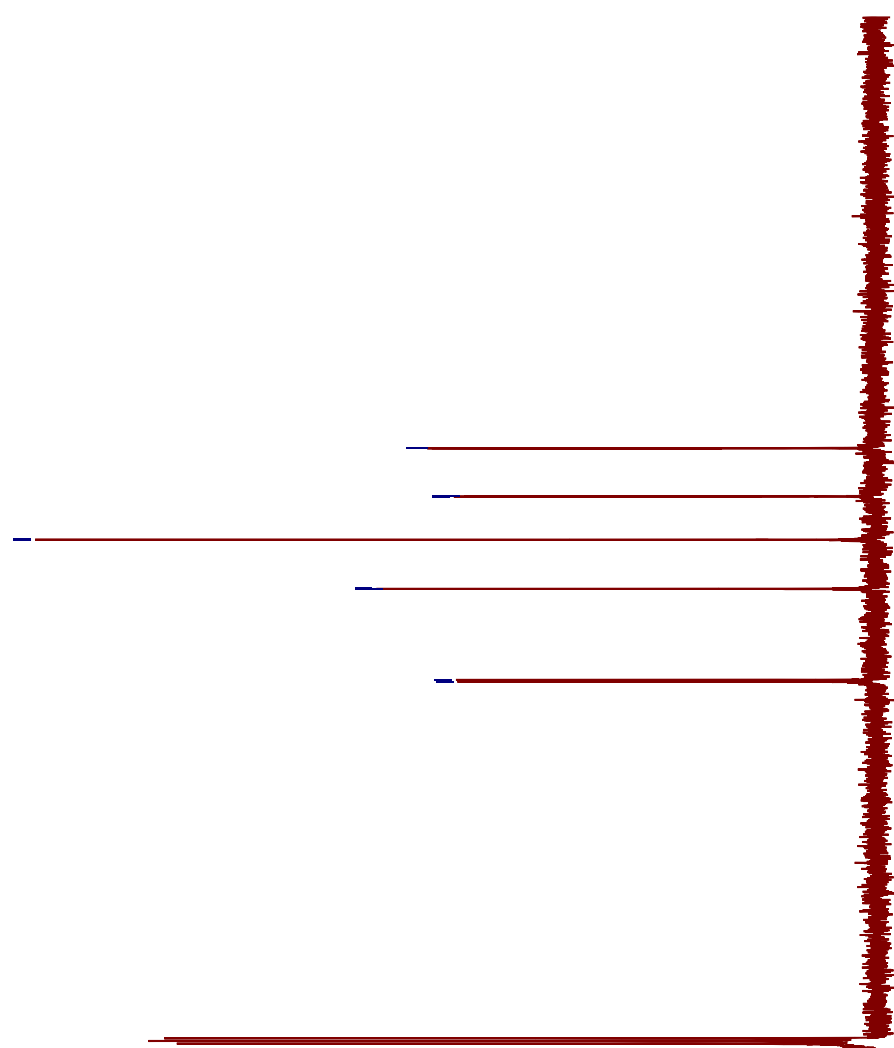


Bioorthogonal retro-Cope elimination reaction of N,N-dialkylhydroxylamines and strained alkynes Dahye Kang and Justin Kim*

乙'ZLI--

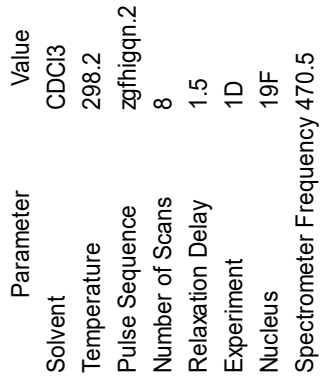

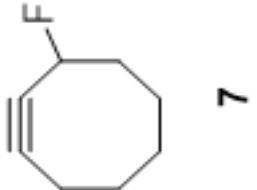


Bioorthogonal retro-Cope elimination reaction of N,N-dialkylhydroxylamines and strained alkynes Dahye Kang and Justin Kim*
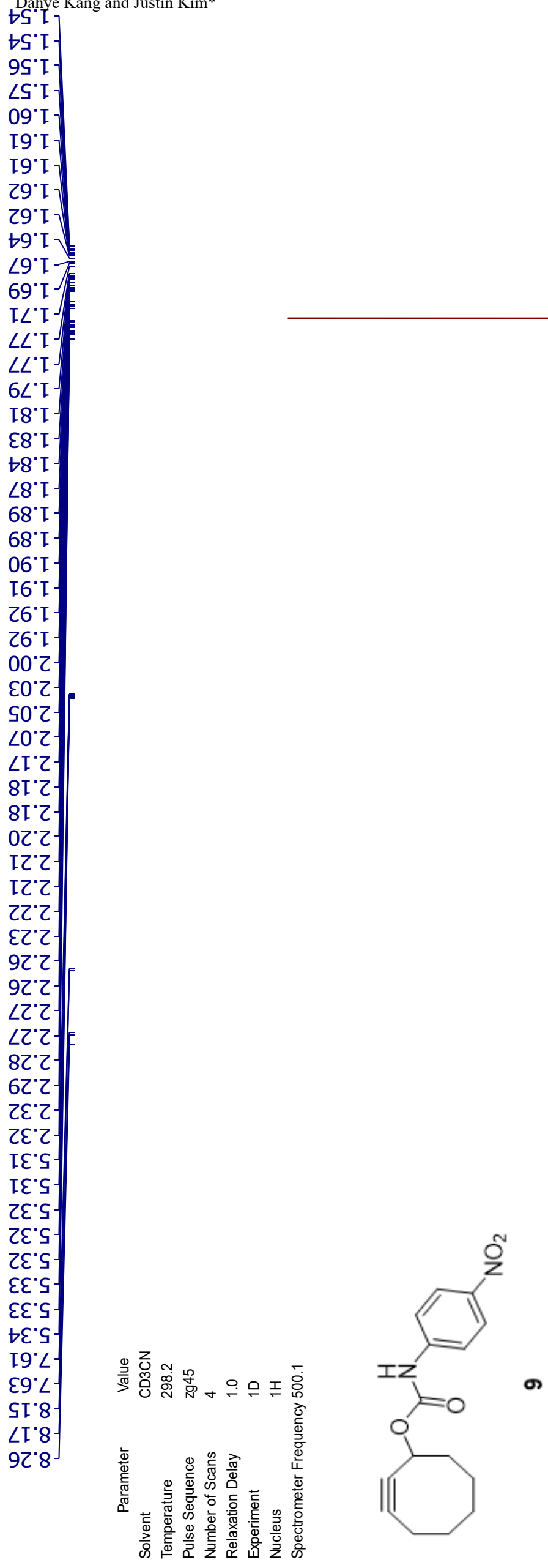

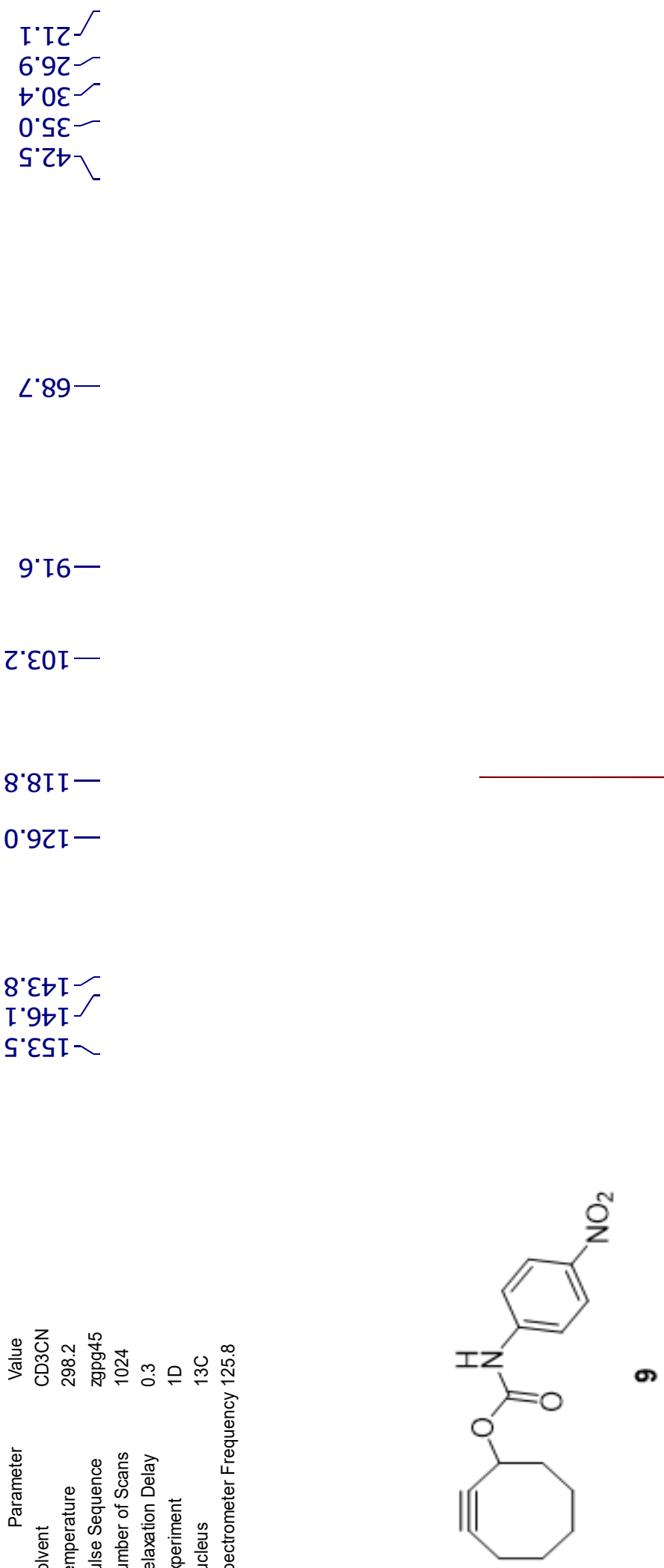

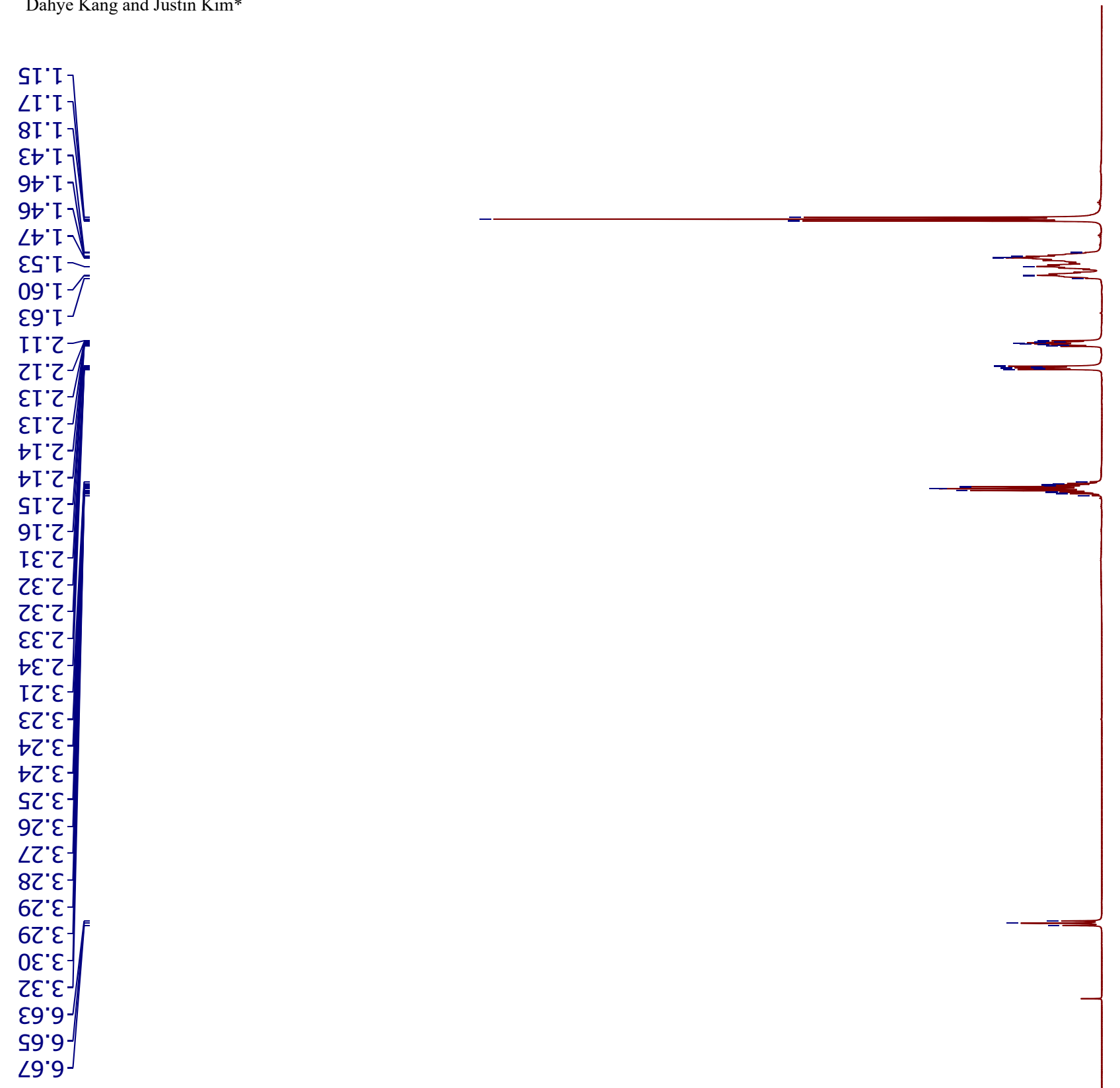

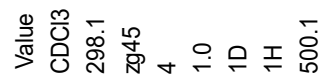

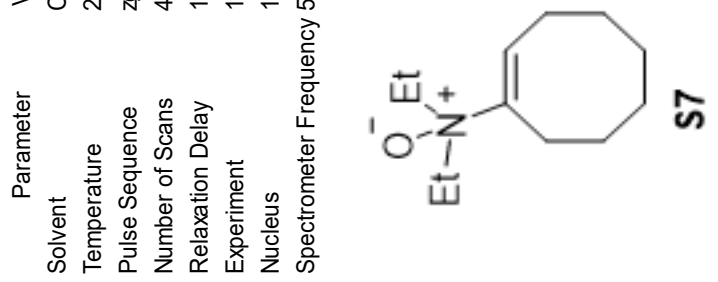




\section{$8 \cdot 8-$ \\ $\varepsilon^{\prime} S Z_{7}$ \\ $9.9 Z$ \\ $0.92 \div$ \\ I.92] \\ $\varepsilon^{\cdot} 8 \tau^{-}$ \\ $\angle \cdot 6 \tau^{\prime}$}

8. $19-$

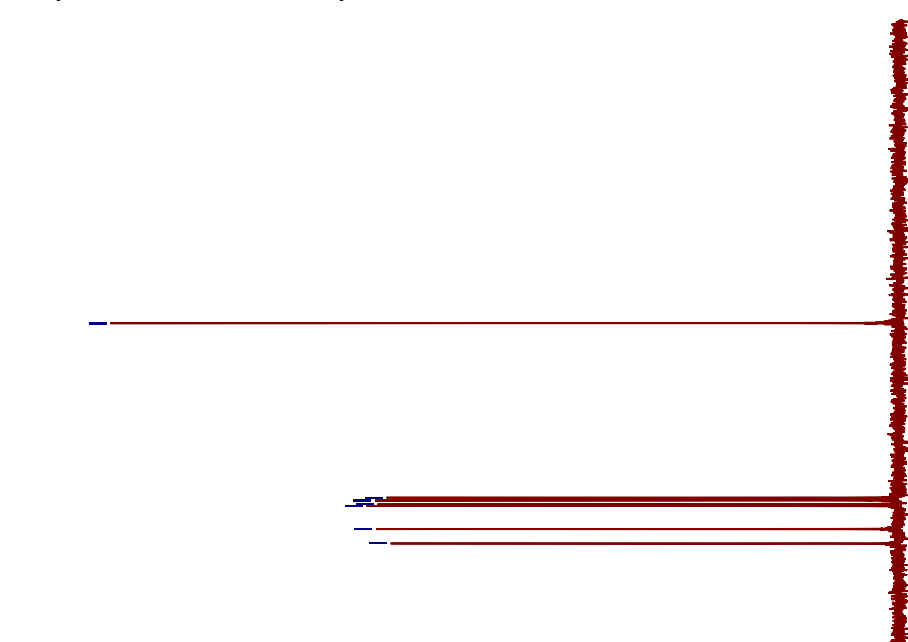

产 旁

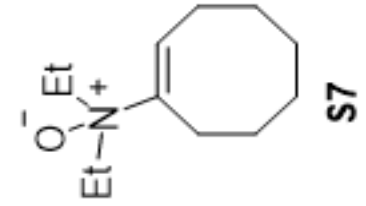


EO" $\mathrm{L}$

$90^{\circ} \mathrm{I}$

$90 . \mathrm{T}$

$60 . \mathrm{T}$

ZI' I

$\varepsilon I^{\cdot} \mathrm{I}$

$\left.\angle I^{\prime} \mathrm{T}\right]$

$6 I^{\prime} \cdot \mathrm{I}$

$0 Z \cdot \tau-j$

$0 \mathrm{Z}^{\prime} \mathrm{I}$

IZ' [ ]

ZZ' I

ZZ' I

$8 S^{\prime} \mathrm{I}$

โ9. I

ह9. I

เ9. I

99. I

69' I

ZL' I

SO $\mathrm{C}$

$80^{\circ} \mathrm{Z}$

OI' $\mathrm{Z}$

$\varepsilon I^{\prime} Z$

tI' $Z$ -

$\angle I ' Z$ -

6I' 2

$0 Z$ '

IZ'Z

$t z^{\prime} z$

$t t^{\circ} \mathrm{C}$

St 2

$\angle t \cdot Z$

$8 b^{\circ} 2$

$0 S^{\prime} \mathrm{Z}$

हS' 2 -

09' 2

โด' 2

$+9{ }^{\circ} \mathrm{Z}$

$99^{\circ} \mathrm{Z}$

$\varepsilon \varepsilon^{\prime} \varepsilon-$

ऽ $\varepsilon^{\prime} \varepsilon-$

$6 \varepsilon^{\prime} \varepsilon-$

$\downarrow t^{\circ} \varepsilon$

$9 t^{\circ} \varepsilon$

$\angle \nabla^{\circ} \varepsilon$

S ${ }^{\circ} \varepsilon$

$9 S^{\circ} \varepsilon$

$65^{\circ} \varepsilon$

$09^{\circ} \varepsilon$

โ9' $\varepsilon$

$\downarrow 9^{\circ} \varepsilon$

$\succ 9^{\circ} \varepsilon-$

ธو' $\varepsilon$

$99^{\circ} \varepsilon$

$89^{\circ} \varepsilon$

ع9.9-

ร9.9

$\angle 9^{\circ} 9$

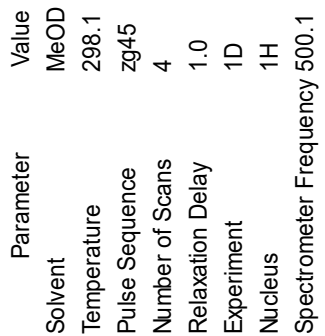

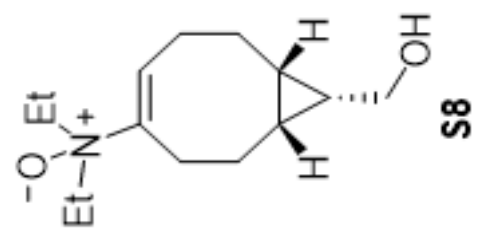

展

产 $\varepsilon 0^{\circ}$

r

뭉

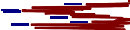

†I:9

- 눈

$-80.2$

-TIOI

${ }^{50} \mathrm{C}$

F-90'z- 눈

Ћ ع0. I
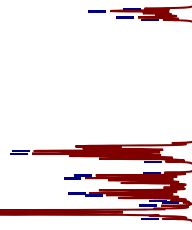
Bioorthogonal retro-Cope elimination reaction of N,N-dialkylhydroxylamines and strained alkynes

Dahye Kang and Justin Kim*
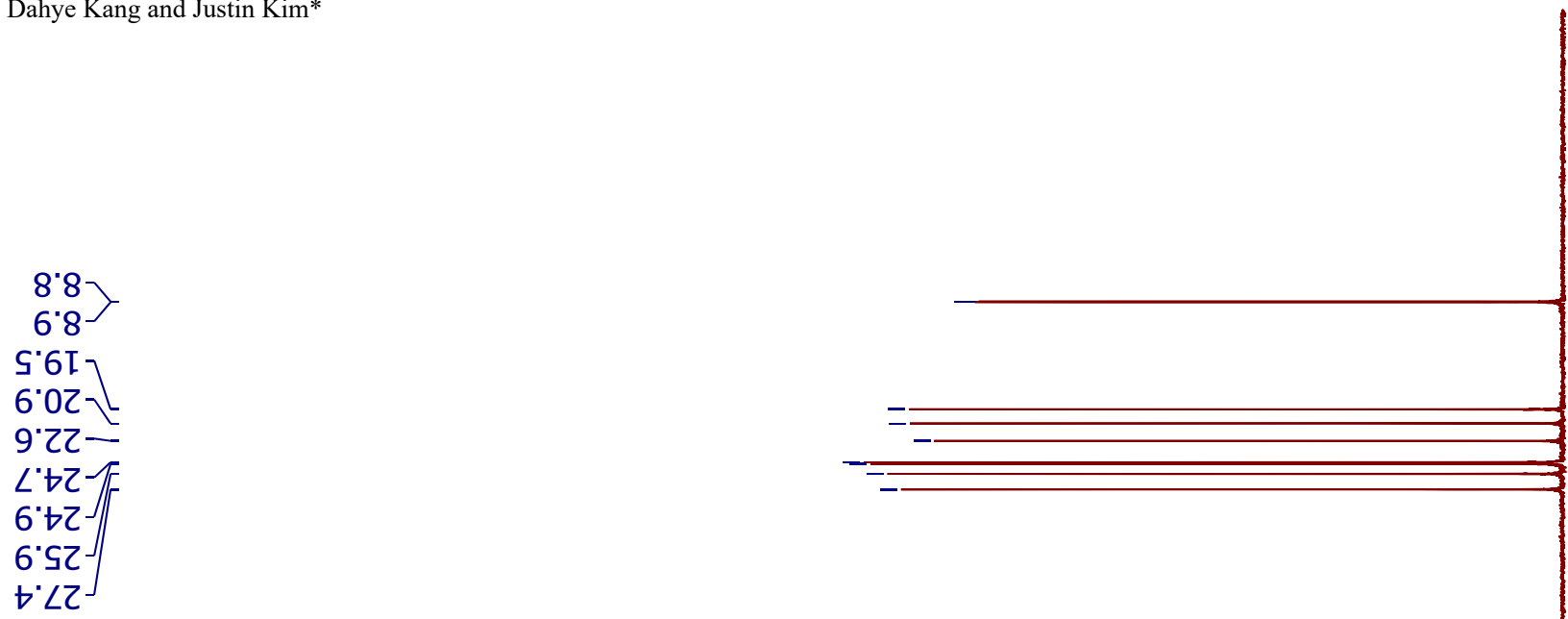

$\angle \cdot 6 S$ ר

$\left.\begin{array}{l}\angle 2 \\ 8.29\end{array}\right\rangle$

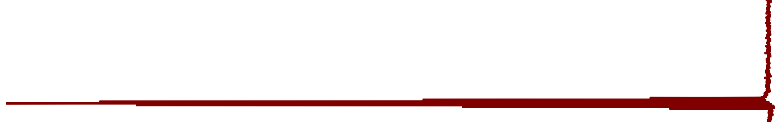

$829^{-1}$

$S^{\prime} \angle Z I-$

$t^{\circ} 8 t I-$

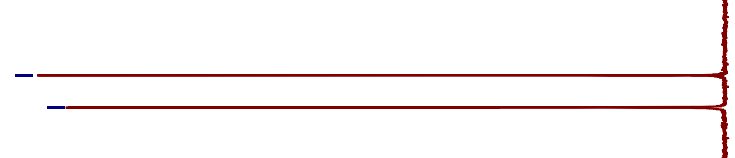

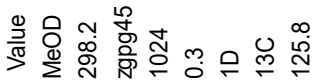
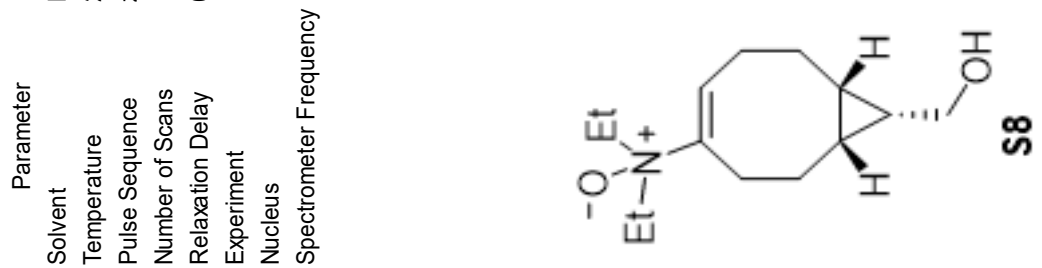

유 


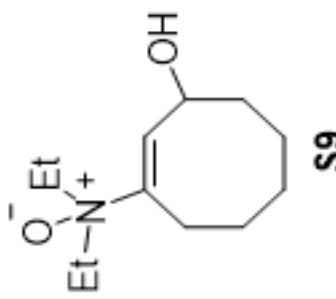



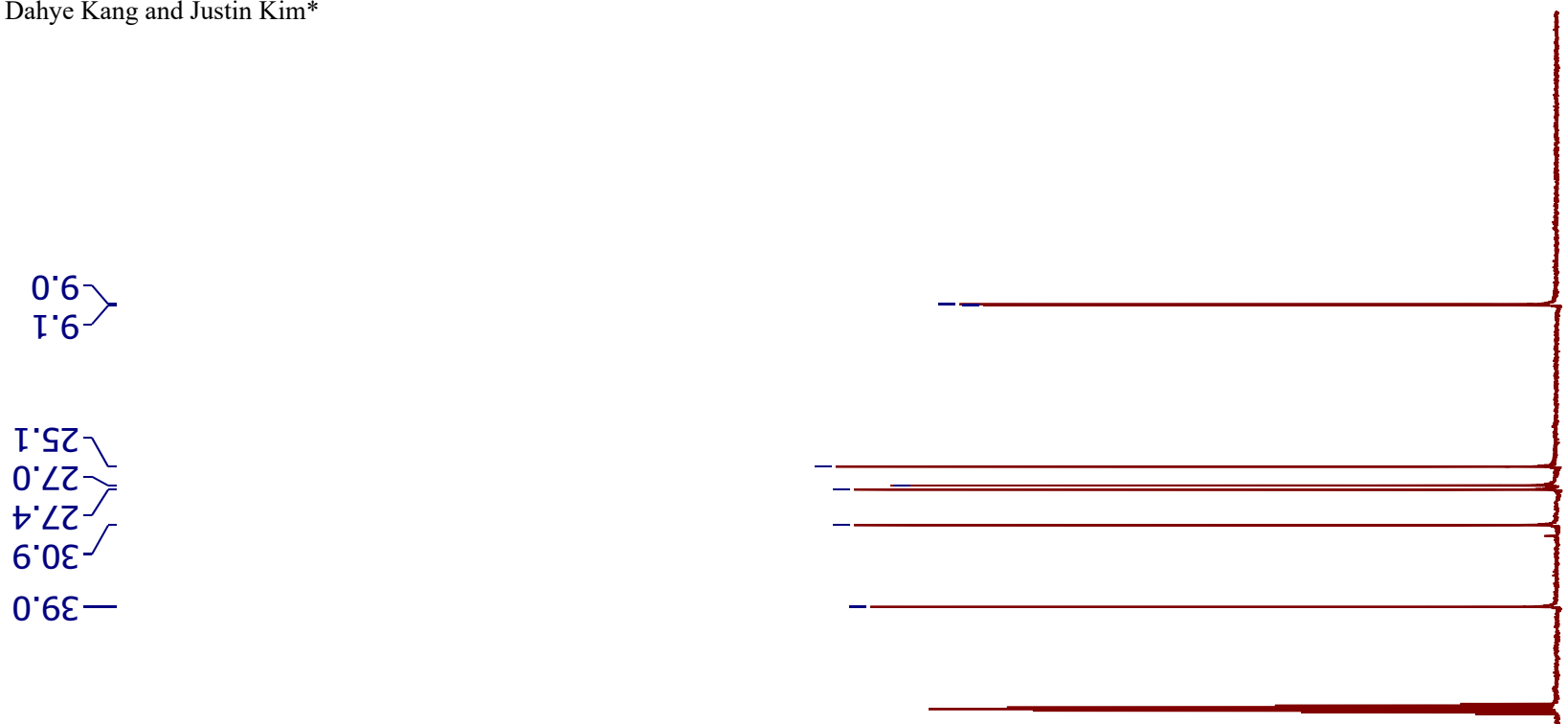

8. โ9-

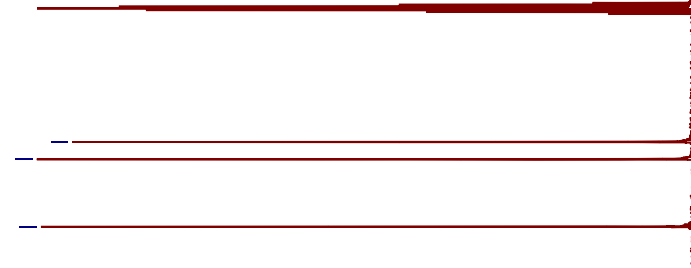


$6 I^{\prime \prime} \mathrm{I}$

IC'I

ZZ' I

†9' I

S9. I

S9. I

99. $\mathrm{T}$

$\angle 9^{\circ} \mathrm{I}$

$\angle 9^{\circ} \mathrm{I}$

$89^{\circ} \mathrm{I}$

69' I

69. I

$0 \angle \mathrm{T}$

IL'T

ZL'I

$b L^{\prime} \mathrm{I}$

$S L ' I-$

9L' I

$26^{\circ} \mathrm{I}-$

$26^{\circ} \mathrm{I}$

ह6" $\mathrm{I}$

†6 I

$\angle L ' Z$

8L'乙-

6L'乙

$\downarrow \varepsilon \cdot \varepsilon$

५ร' $\varepsilon$

५દ' $\varepsilon$

$9 \varepsilon$ ' $\varepsilon$

$\angle \mathcal{E}^{\prime} \varepsilon$

$8 \varepsilon^{\prime} \varepsilon$

$8 \varepsilon^{\prime} \varepsilon$

$6 \varepsilon^{\prime} \varepsilon$

โ七 $\varepsilon$

$\angle b^{\circ} \varepsilon$

$8 t^{\prime} \varepsilon-$

$6 t^{\circ} \varepsilon$

$6 b^{\prime} \varepsilon$

OS' $\varepsilon$ -

IS' $\varepsilon$

乙S' $\varepsilon$

$\varepsilon S^{\prime} \varepsilon$

$68^{\circ} \varepsilon-$

$06^{\circ} \varepsilon$

$06^{\circ} \varepsilon$

โ6' $\varepsilon$

โ6 $\varepsilon$

โ6 $\varepsilon$

$26^{\circ} \varepsilon$

$\varepsilon 6^{\circ} \varepsilon$

$\rightarrow 6^{\circ} \varepsilon-$

$\mathrm{S} 6^{\circ} \varepsilon$

ธ6 $\varepsilon$

$56^{\circ} \varepsilon$

$96^{\circ} \varepsilon$

$\angle 6^{\circ} \varepsilon$

$\angle 9^{\circ} 9^{\circ}$
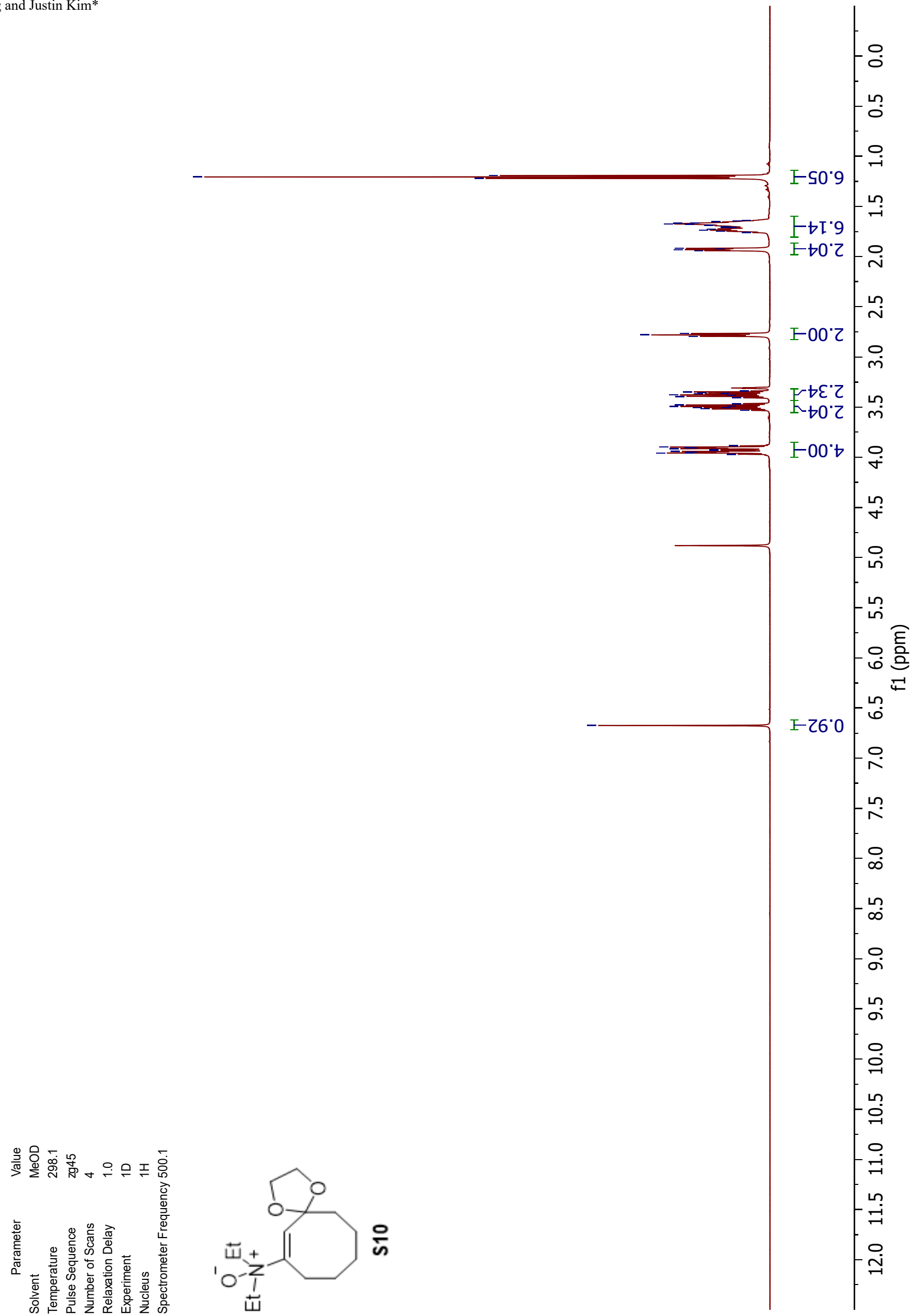
Bioorthogonal retro-Cope elimination reaction of N,N-dialkylhydroxylamines and strained alkynes Dahye Kang and Justin Kim*

$$
0.6-
$$

$\left.9^{\circ} \varepsilon Z\right]$

$$
\begin{array}{ll}
0.95 \\
0.65 & \frac{1}{2}
\end{array}
$$

6.62

9.0t-

โ.ع9-

เ's9-

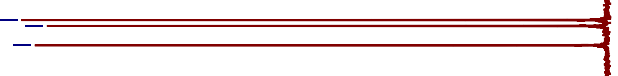

$-$

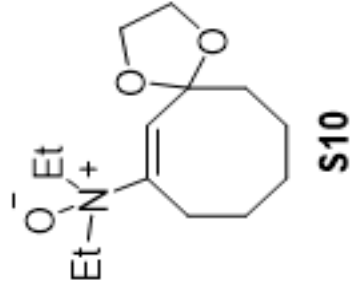


โt. 9
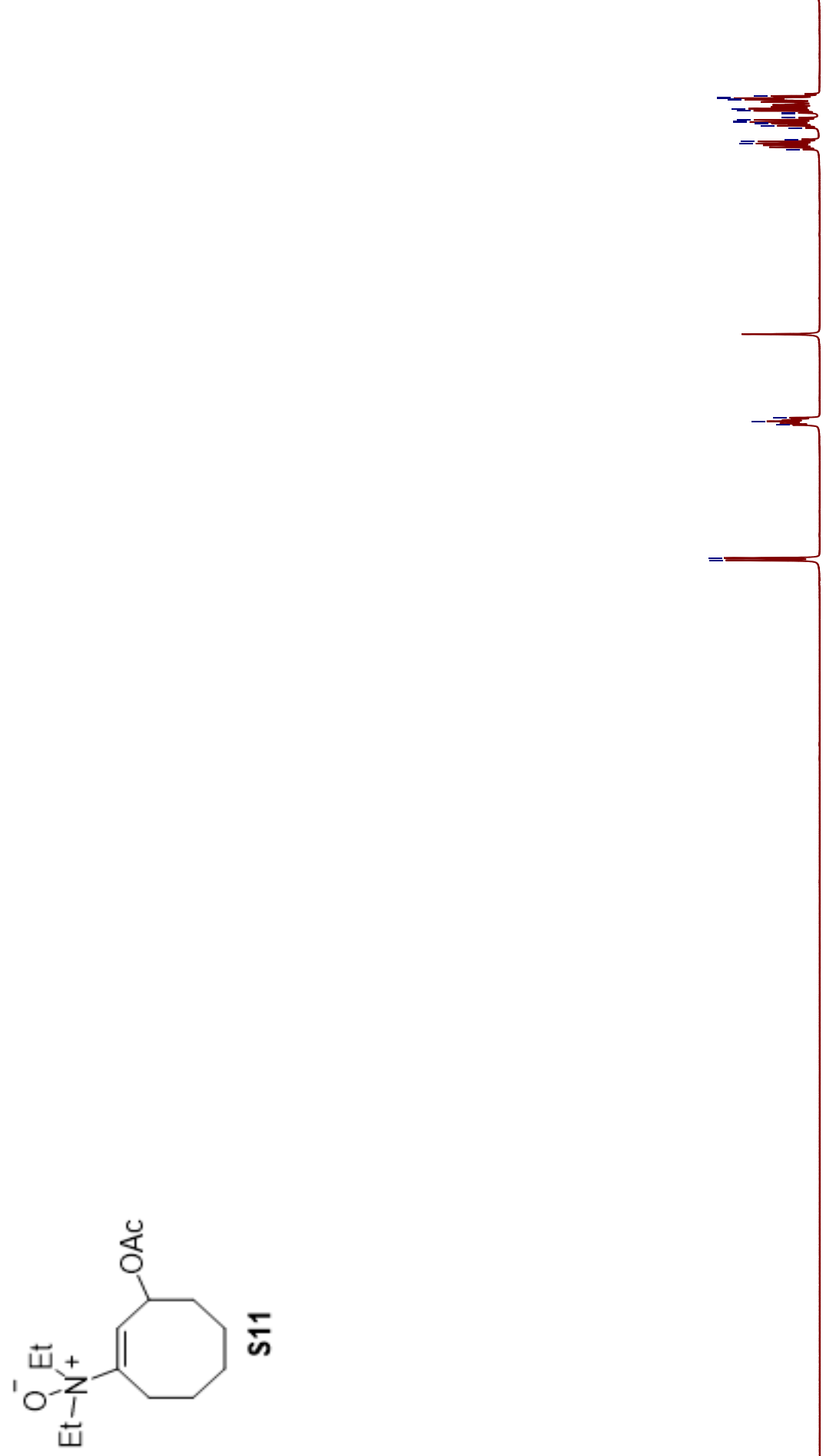

要

F $\frac{T}{T} Z I^{\circ}$

웅

К $2 I$ ' $\stackrel{n}{m}$

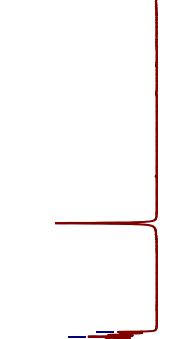

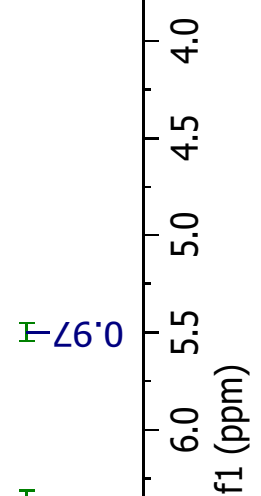
-00 ' I

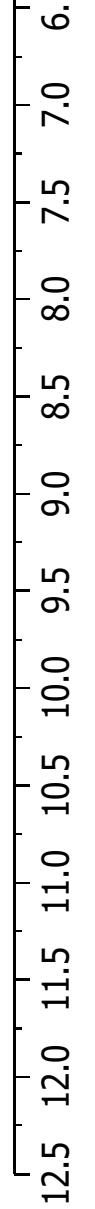




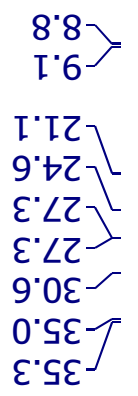

I'Z9>

$9^{\circ} \varepsilon 9^{-1}$

$乙^{\cdot} \varepsilon<-$
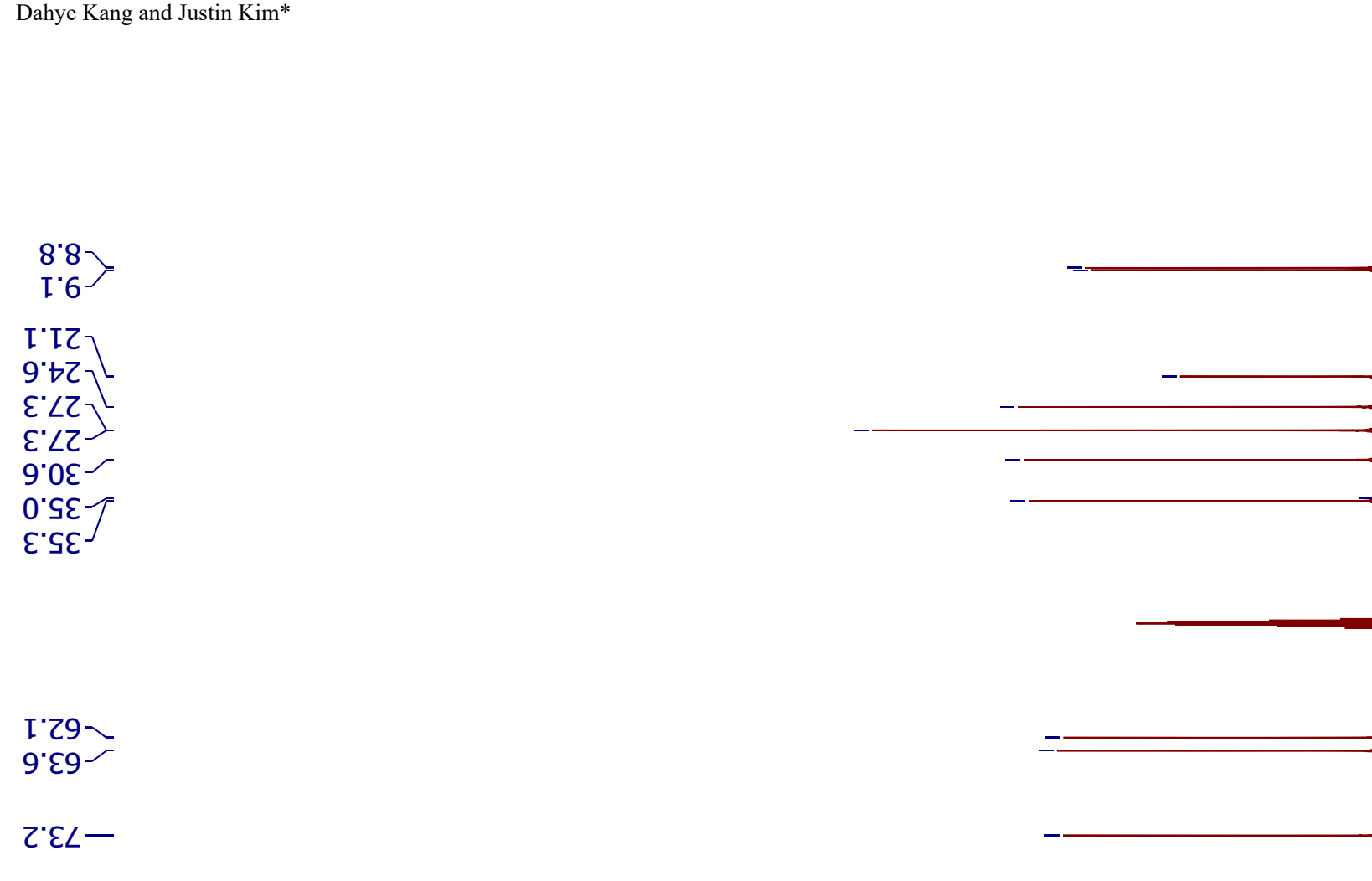

†.8ZI-

$8^{\circ} \angle t I-$

Z'ZLI-

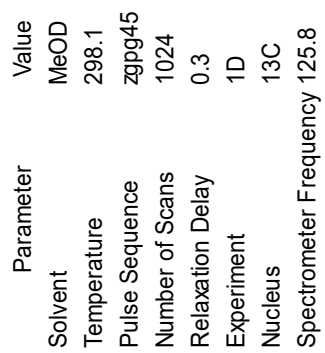

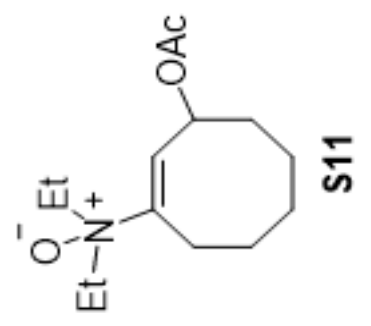



$\angle 9^{\circ}{ }^{\circ}$
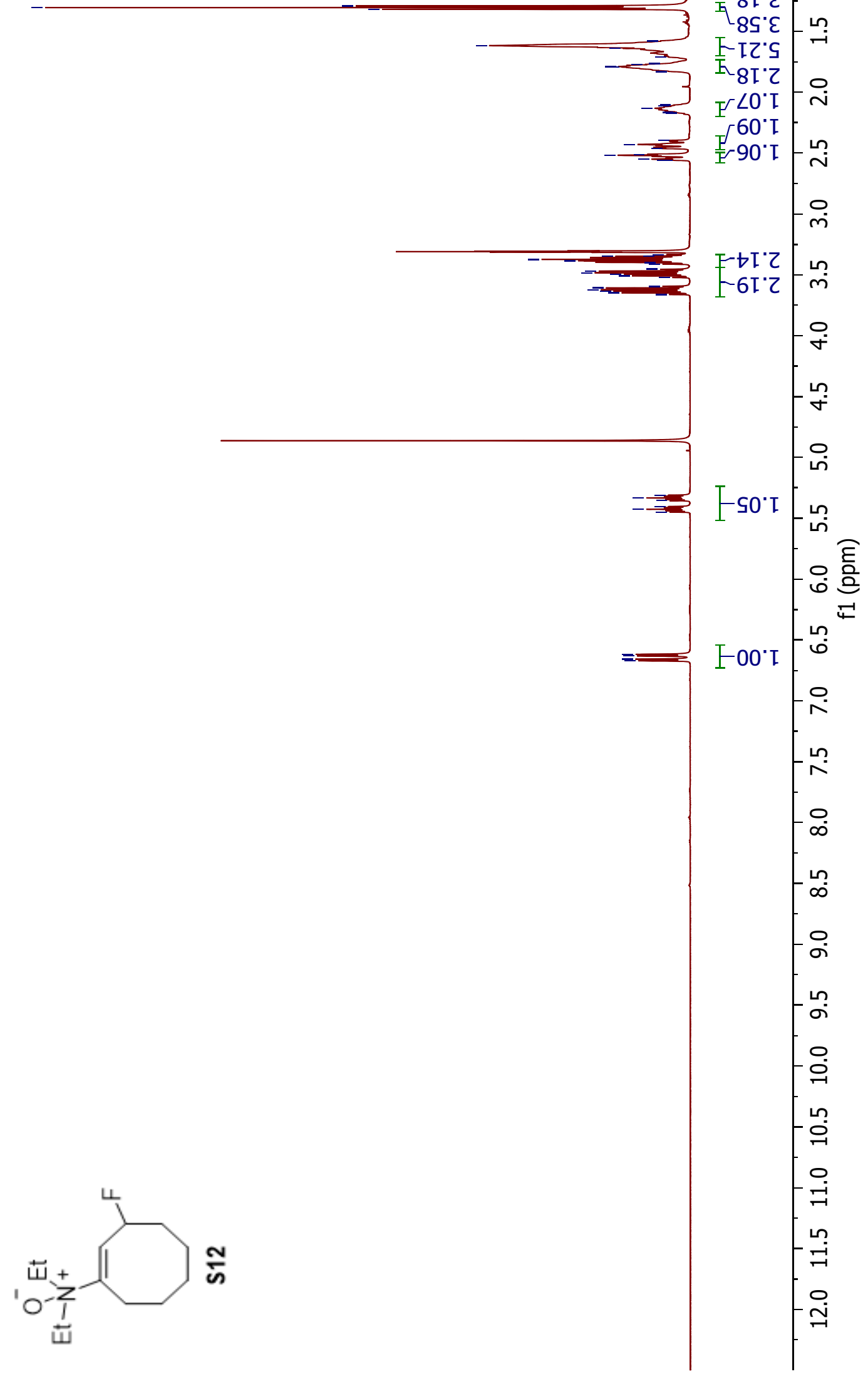
Bioorthogonal retro-Cope elimination reaction of N,N-dialkylhydroxylamines and strained alkynes Dahye Kang and Justin Kim*

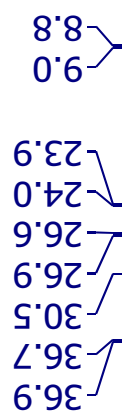

I'Z9

$\angle \cdot \varepsilon 9-$

t.16八

$\angle{ }^{\prime} \mathrm{Z} 6^{\prime}$

$\left.\begin{array}{l}\angle 82 I \\ 0.62 I\end{array}\right\rangle$

$\left.\begin{array}{l}\varepsilon^{\prime} \angle t I \\ b^{\prime} \angle t I\end{array}\right\rangle$

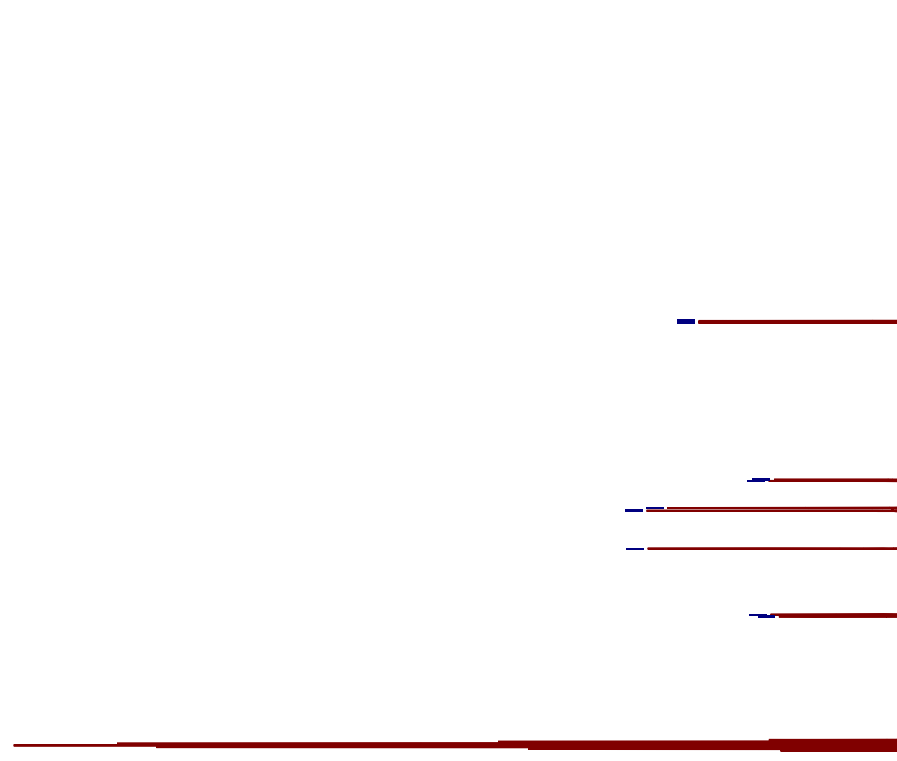

0621

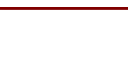

으

$+$

$-0$

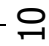

- 요

요

우

으

$-8$

ㅇ

$\infty$

- 8

응 흥

윽

고

윽

온

윰

$-8$

$\frac{1}{2}$

-

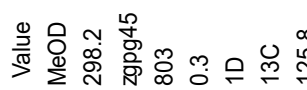

\section{$\stackrel{\infty}{\stackrel{\infty}{\sim}}$}
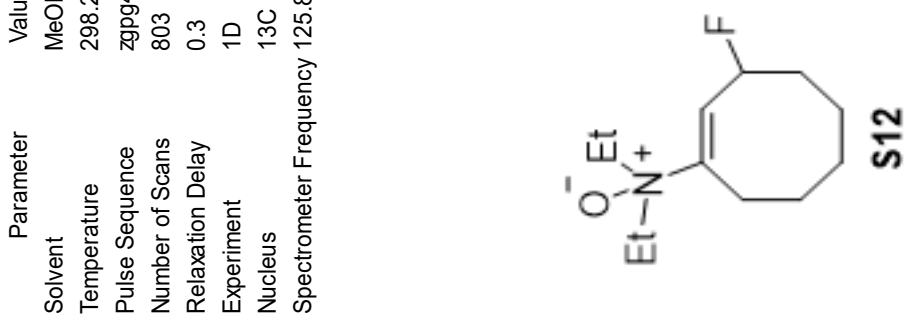

- 욱

유

윽

ิㅡㄴ 
Bioorthogonal retro-Cope elimination reaction of N,N-dialkylhydroxylamines and strained alkynes Dahye Kang and Justin Kim*

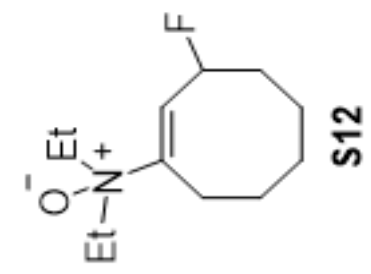



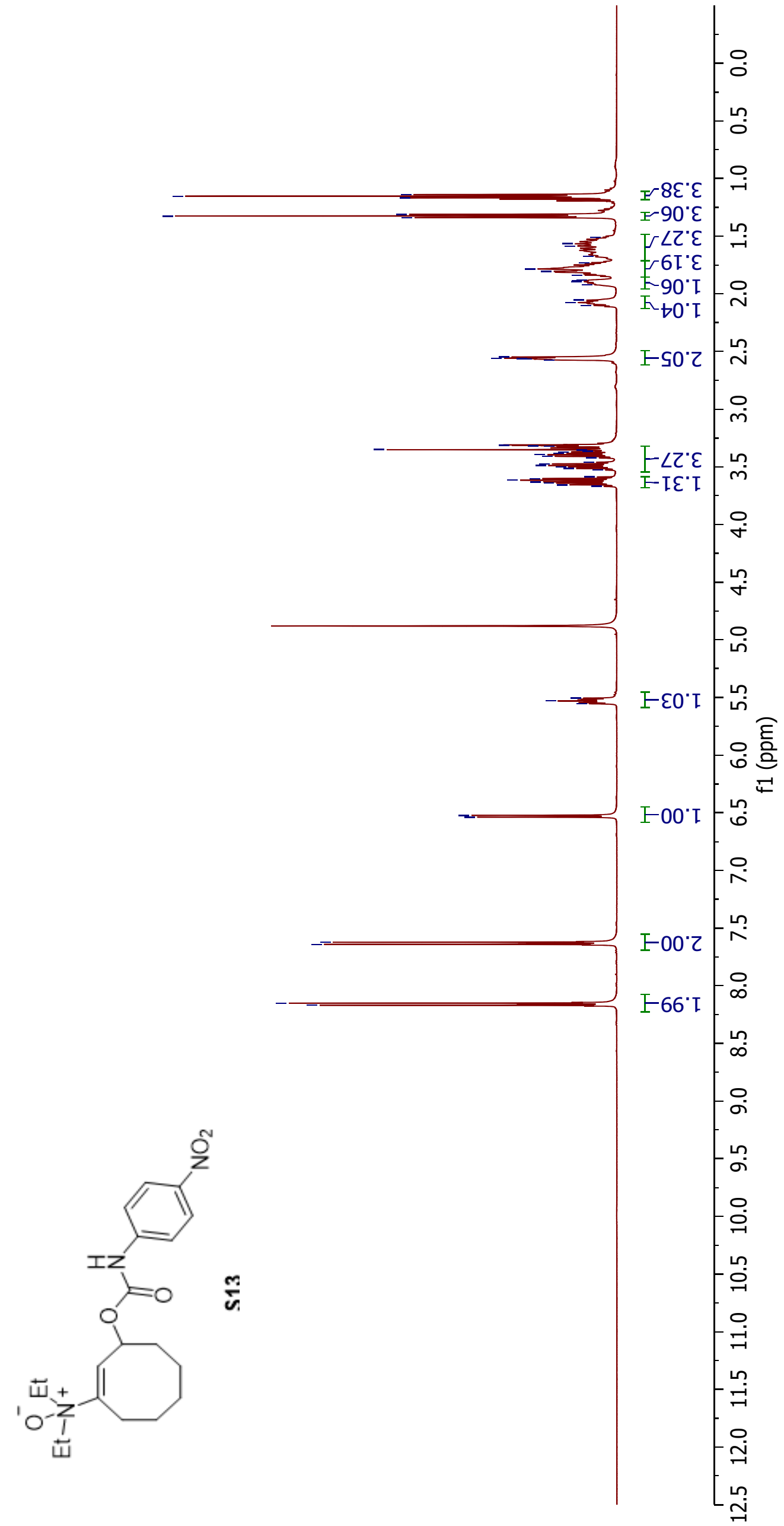
Bioorthogonal retro-Cope elimination reaction of N,N-dialkylhydroxylamines and strained alkynes Dahye Kang and Justin Kim*

6.8
4.6

$9^{*} \bullet 乙 乙$

$\varepsilon^{\prime} \angle Z$
$\varepsilon \cdot \angle Z$
0

$9^{\circ} 0 \varepsilon^{2}$

s's $\varepsilon^{\top}$

乙"Z9

$9^{\circ} \varepsilon 9^{-}$

$I^{\circ} \mathrm{bL}$

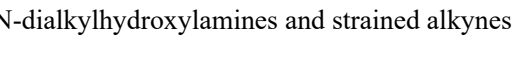

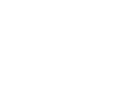

$-$

음

- 요

요

-우

으

$-8$

R

$-\infty$

8

음

윽

ㅇำ

$0.92 \mathrm{I}$

t.82 I -

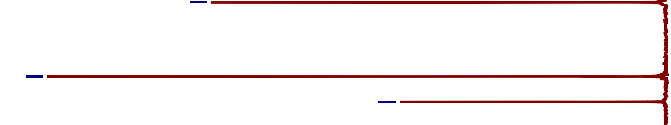

요

- 온

6.E†I-

6.9tI $0.8 b I$

$9{ }^{\circ} \mathrm{SI}$ -

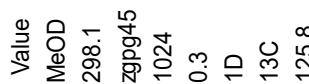

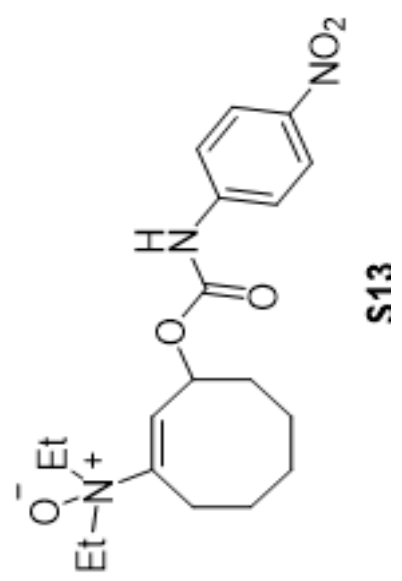

- 윰

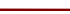

$-8$

요

욤

-

- 움

- 유

윽

공 
Bioorthogonal retro-Cope elimination reaction of N,N-dialkylhydroxylamines and strained alkynes Dahye Kang and Justin Kim*

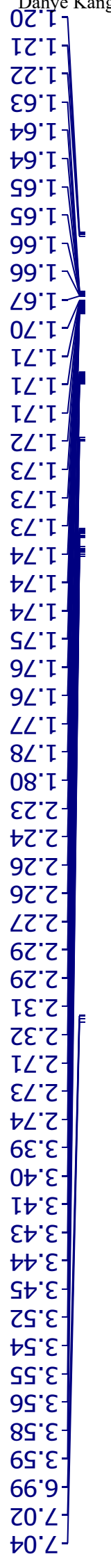
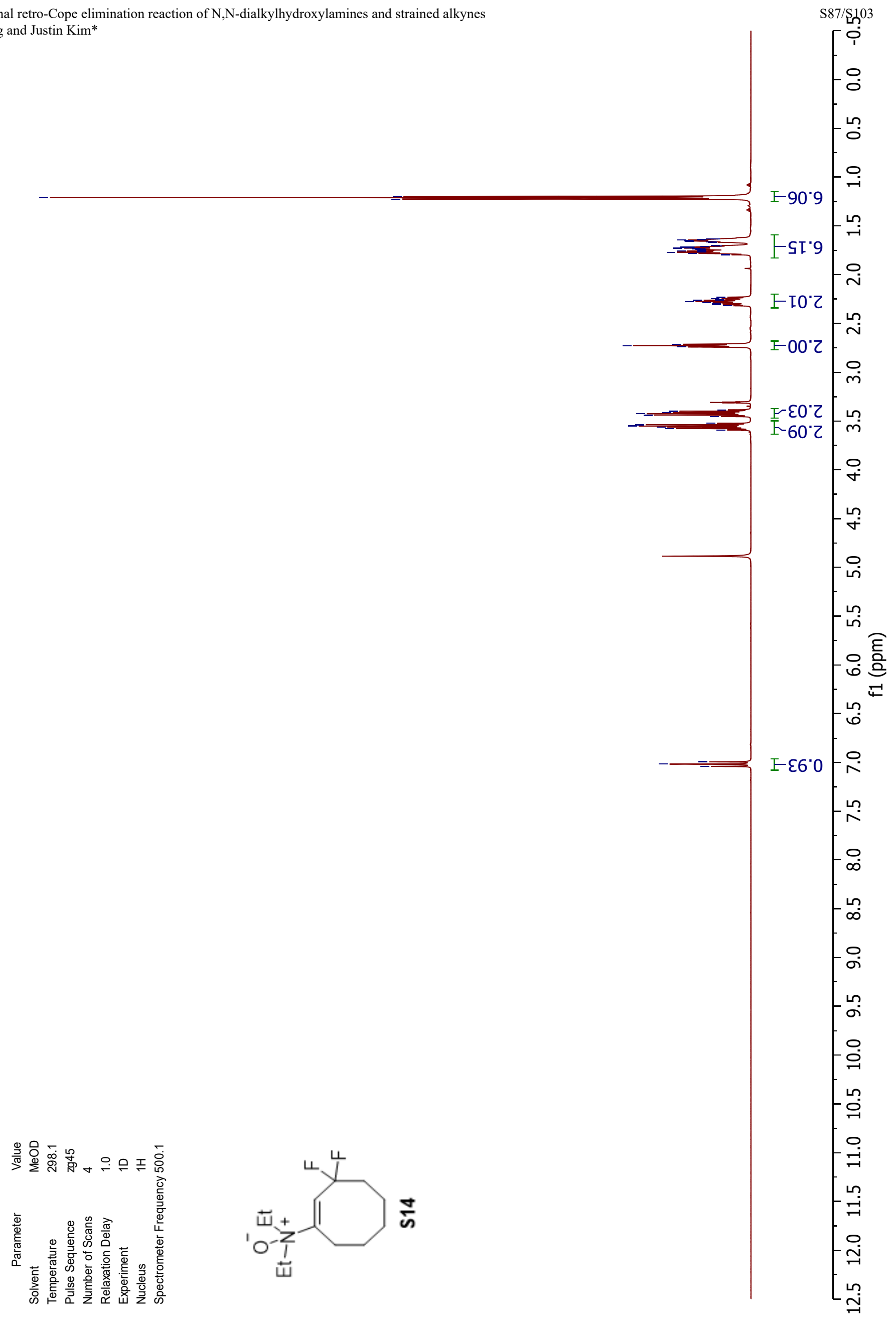
Bioorthogonal retro-Cope elimination reaction of N,N-dialkylhydroxylamines and strained alkynes Dahye Kang and Justin Kim*

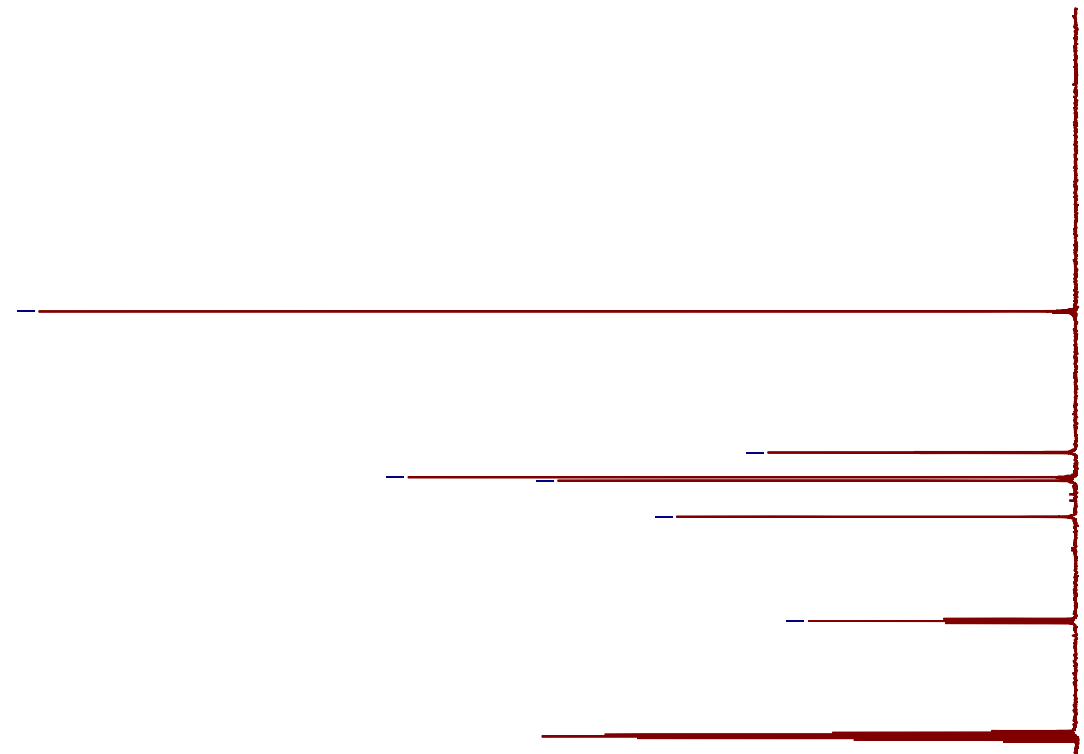

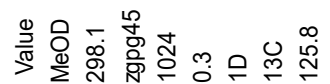

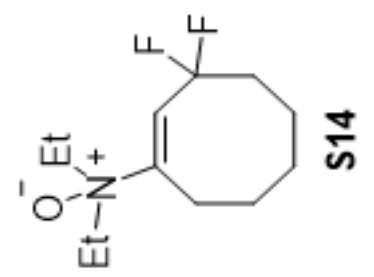




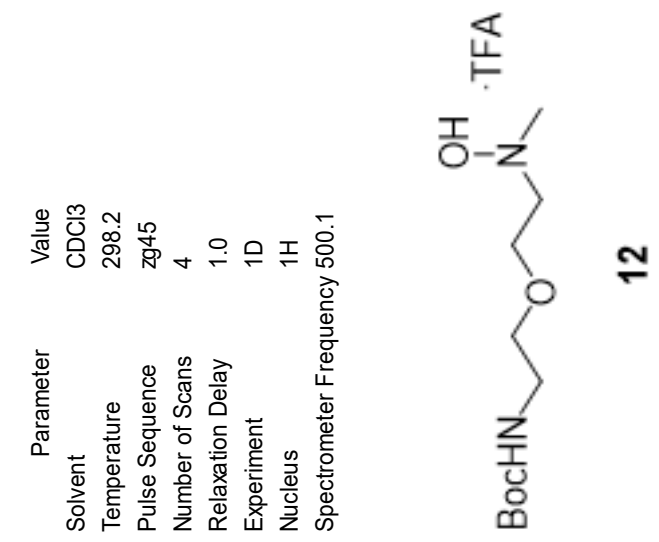


Bioorthogonal retro-Cope elimination reaction of N,N-dialkylhydroxylamines and strained alkynes Dahye Kang and Justin Kim*

$s^{\prime} 82-$

ぢ0เー

s'9t-

乙.09

$9.89^{\top}$

$6{ }^{\circ} 0<>$

s: $6<-$

0 'ยII

$\varepsilon^{\prime} \mathrm{SII} \sim$

$9 \circ \mathrm{LI}-$

6.6II

$\angle$ '9SI

$\angle$ ह9I

0 เ 9[

$\varepsilon \circ 59 I$

9 9เ9[

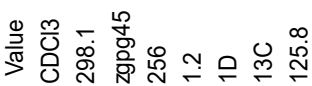

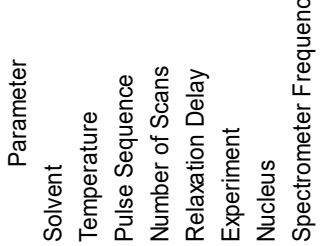

$\stackrel{\amalg}{\rightleftarrows}$

민 
Bioorthogonal retro-Cope elimination reaction of N,N-dialkylhydroxylamines and strained alkynes Dahye Kang and Justin Kim*
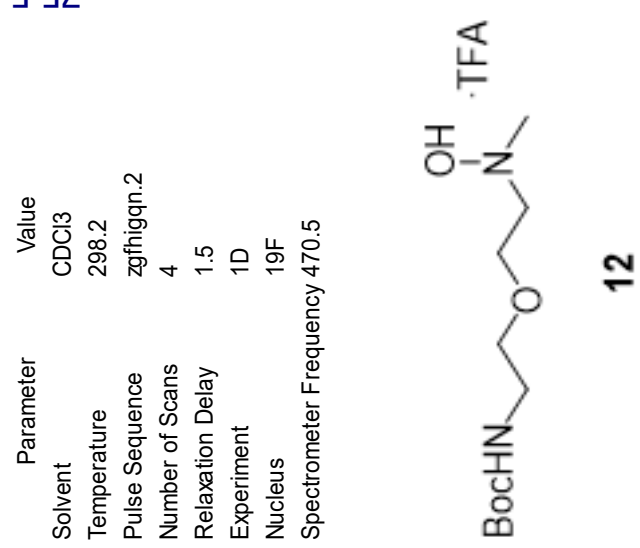


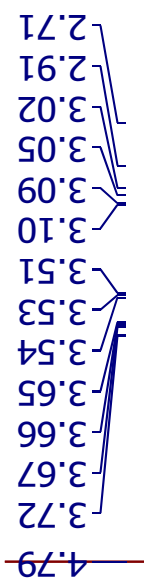

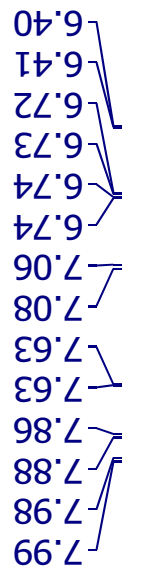

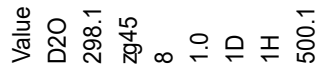
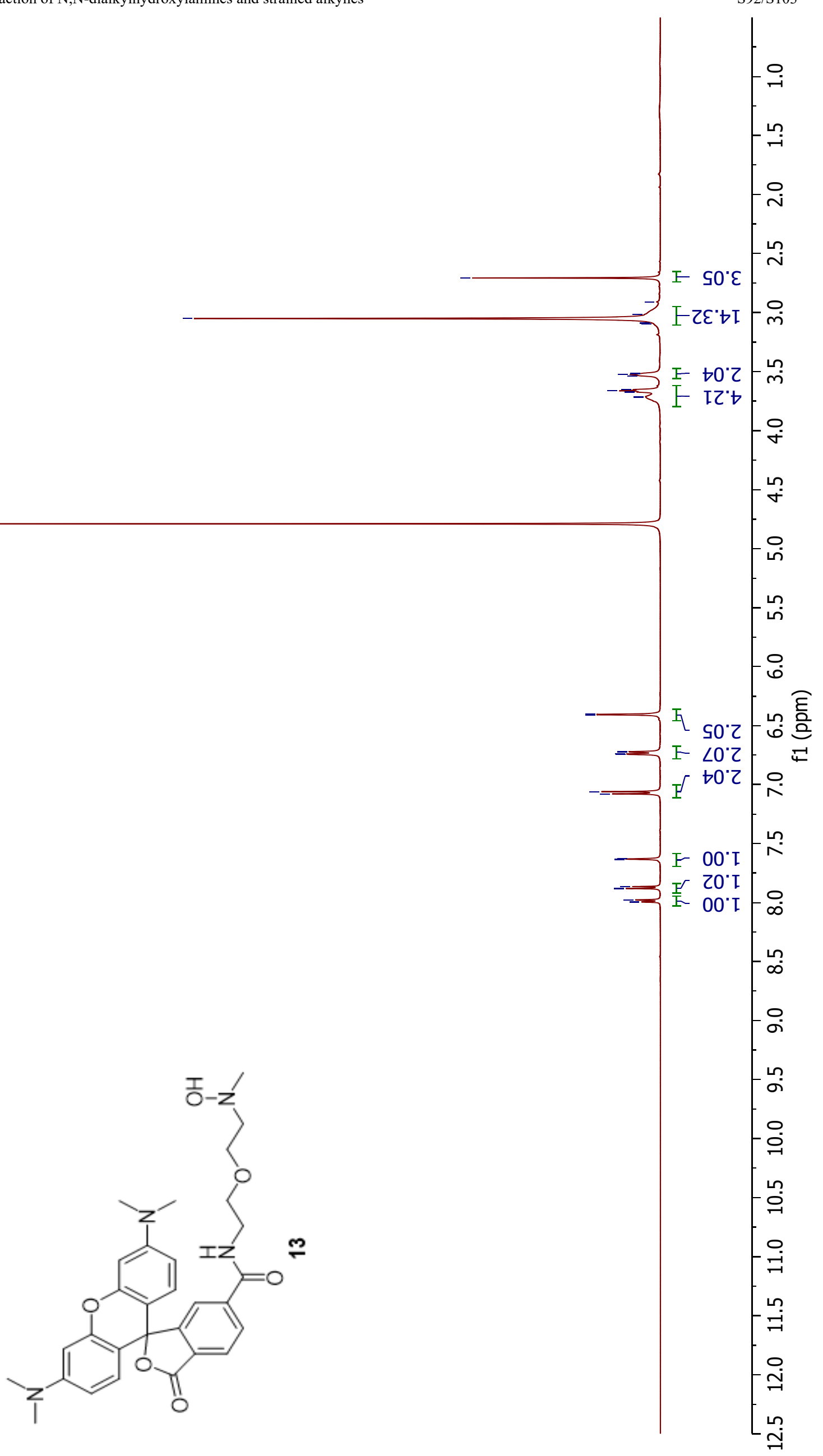
9.9SI

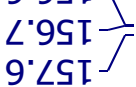

0.89I -

乙'ELI-

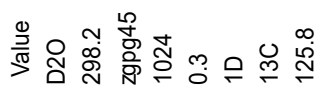<smiles>C=C(CCOCC[C](C)[O-])C(=O)c1ccc2c(c1)C1(OC2=O)c2ccc([C](C)C)cc2Oc2cc([Te](C)(C)C)ccc21</smiles> 
Bioorthogonal retro-Cope elimination reaction of N,N-dialkylhydroxylamines and strained alkynes Dahye Kang and Justin Kim*

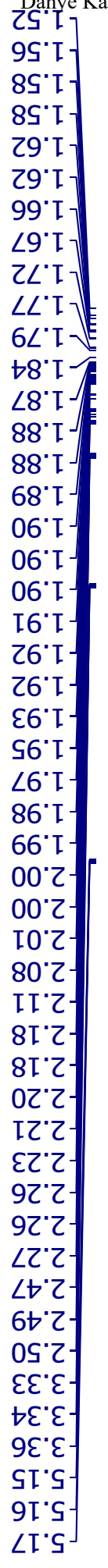
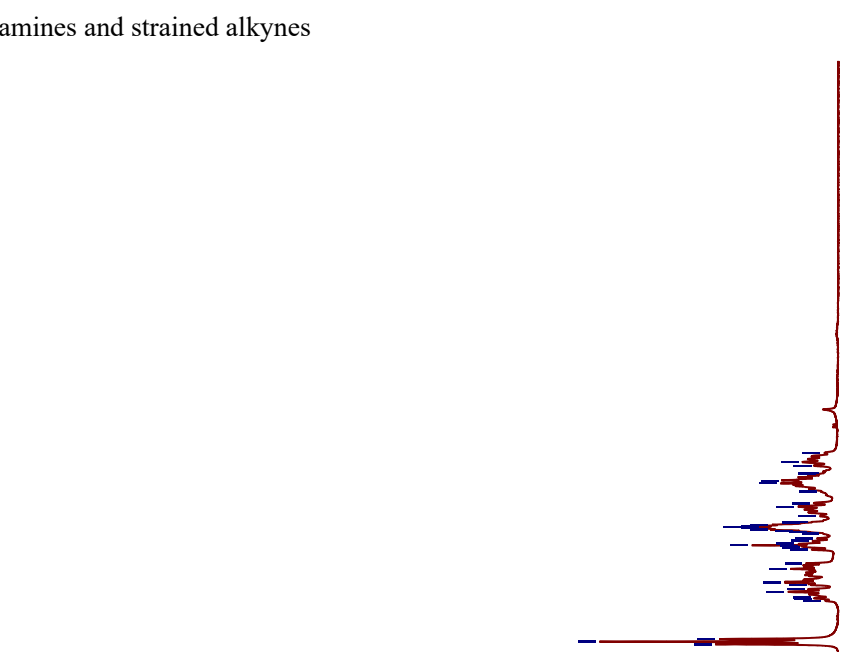

S94/S103

9S. I

$8 S^{\prime} \mathrm{T}$

$89^{\prime} \mathrm{I}$

$29 \cdot$

$=\left[\begin{array}{l}90^{\circ} I \\ \angle I \\ \hline\end{array}\right]-\frac{1}{6}$

$-600^{\circ}$

- II'C

LSO I

$\angle O^{\circ} \mathrm{Z}$

乌66.0

ก

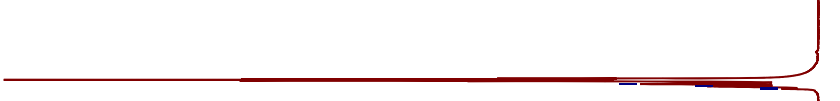

002

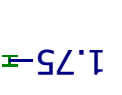

우

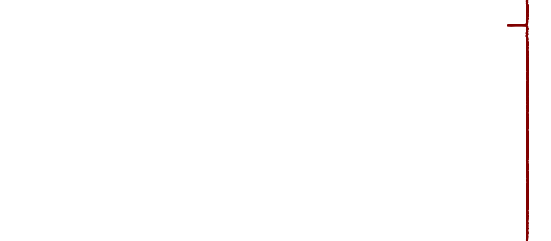

- $86^{\circ} 0$

I

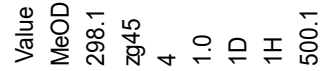
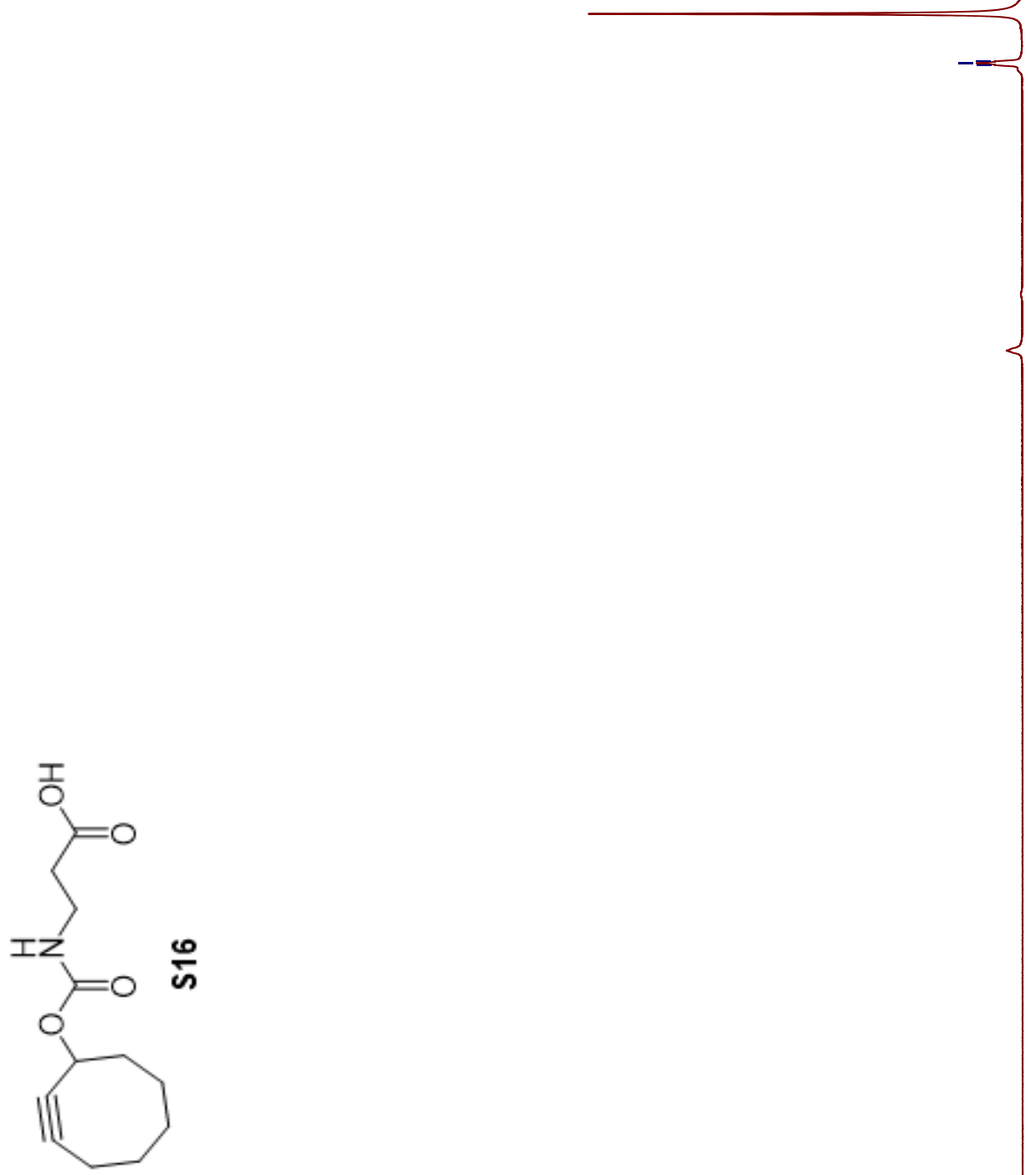
ย'I乙一 七 $^{\prime} \angle \mathrm{C}$ $0^{\circ}[\mathcal{E} \backslash$ $\varepsilon \cdot \varsigma \varepsilon$ S'SE$6^{\circ} \angle \varepsilon^{\prime}$ I'E† -

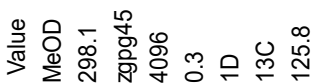

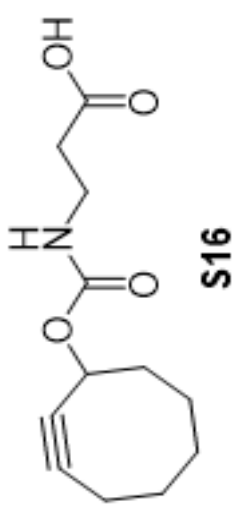



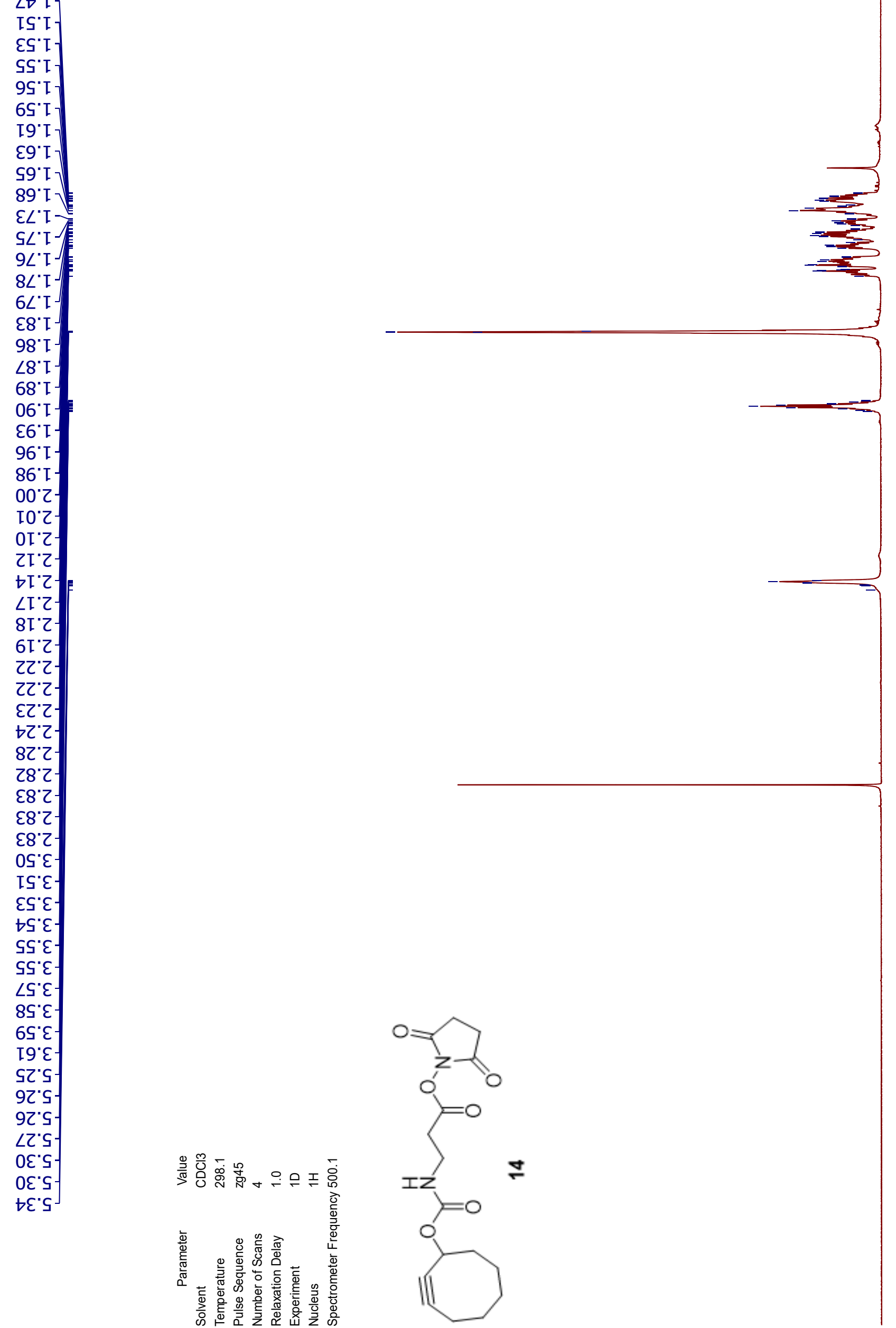
6.02

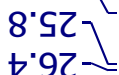

$8.62-$

$\varepsilon \cdot 2 \varepsilon \tilde{\varepsilon}$

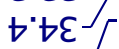

$9.9 \varepsilon$

$0.2 \downarrow^{\top}$

$9 \cdot \angle 9-$

I'I6-

6. TOL -

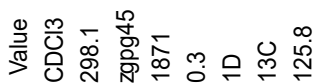
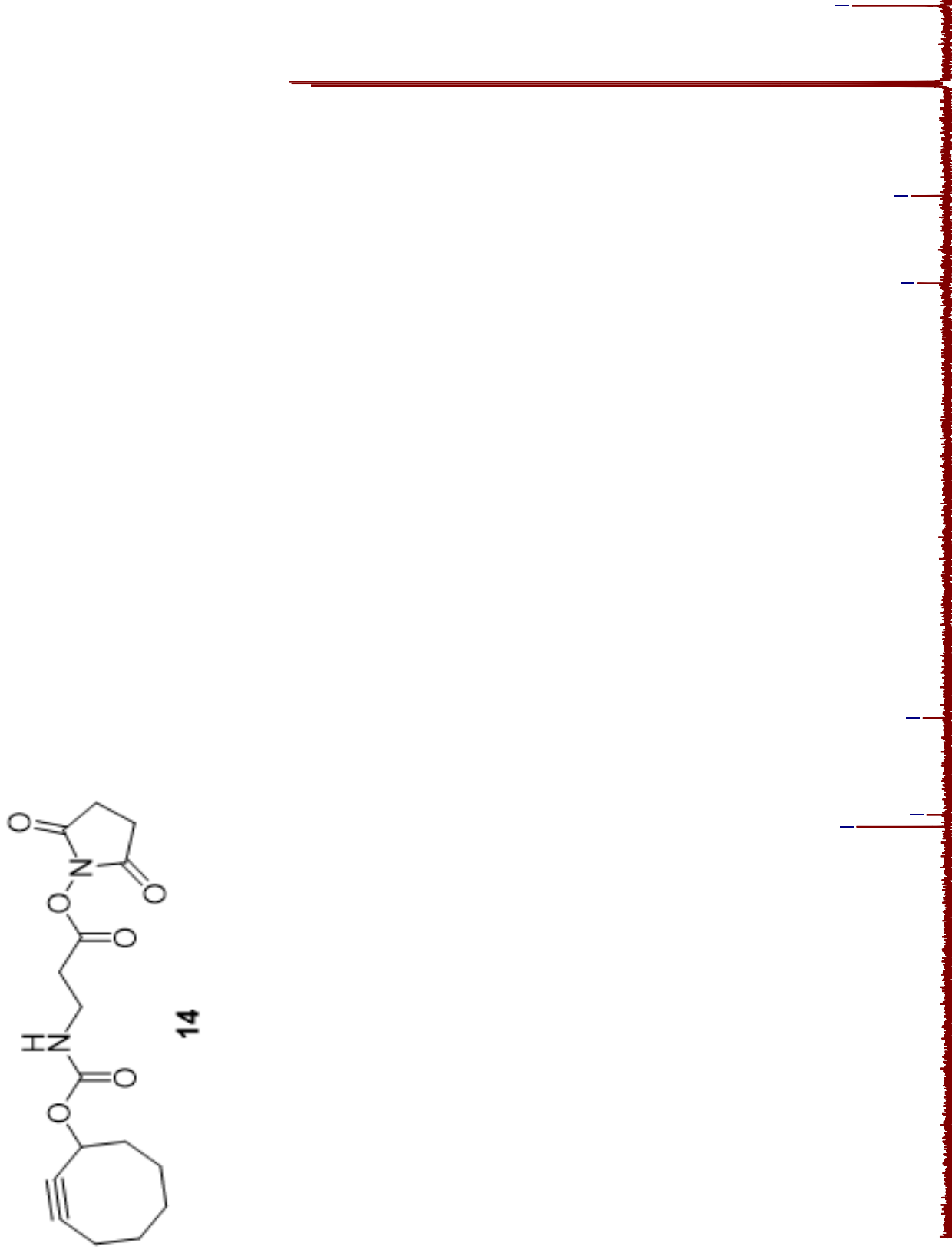

L'SSI -
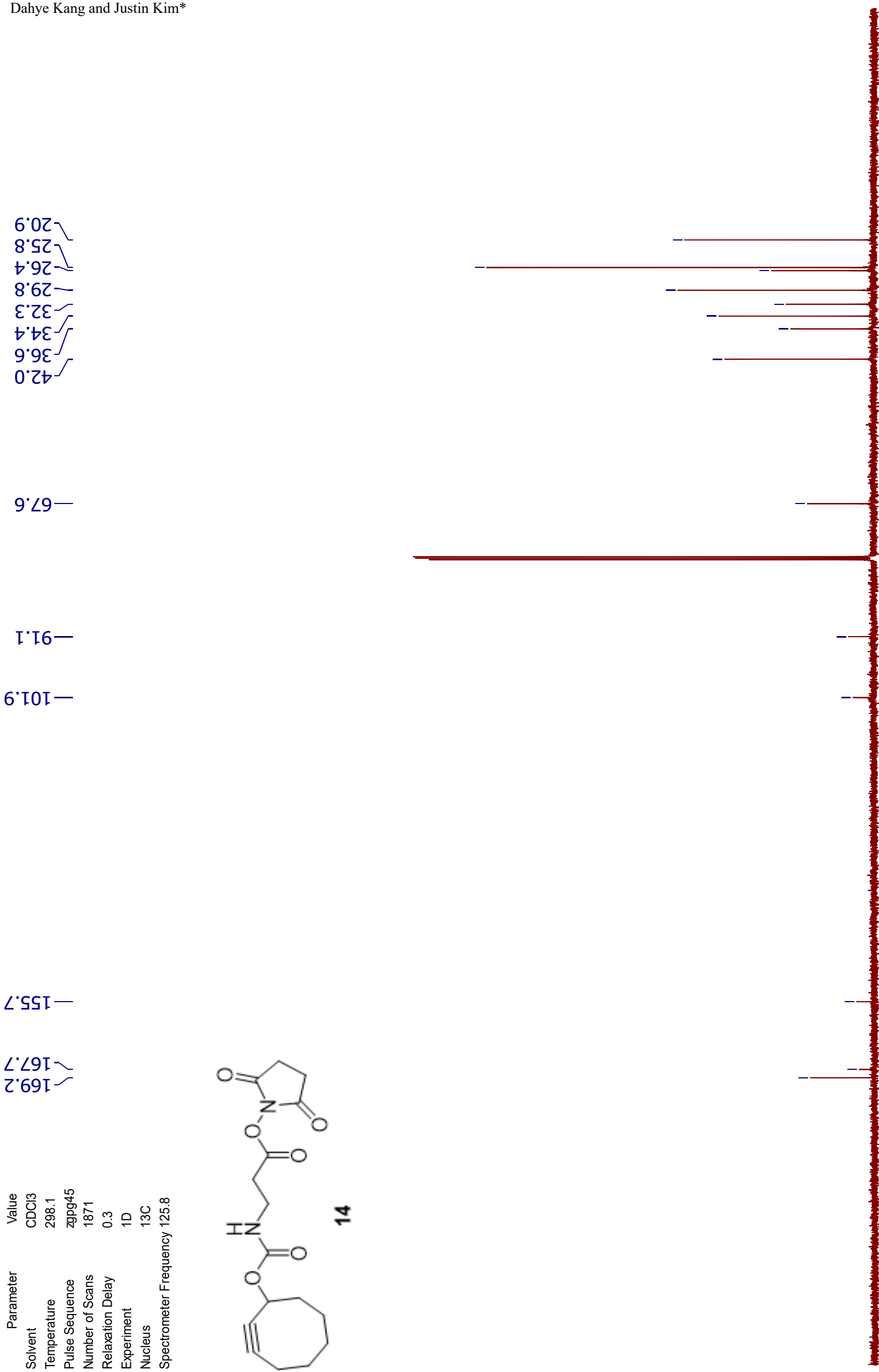
Bioorthogonal retro-Cope elimination reaction of N,N-dialkylhydroxylamines and strained alkynes Dahye Kang and Justin Kim*

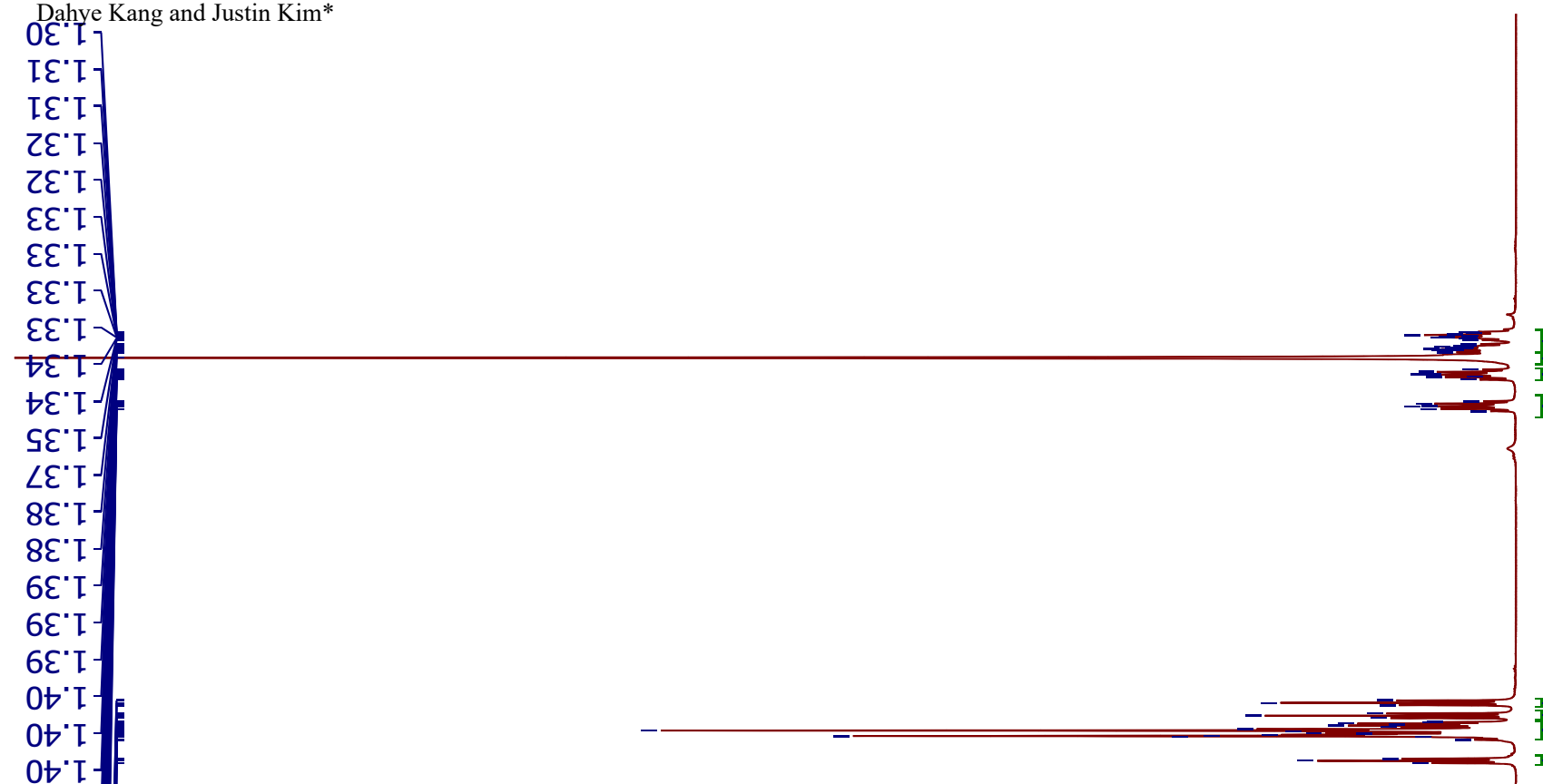

It' I

乙大' I-

St' I-

ZS' I

$\varepsilon S^{\prime} I$

知 $I$

SS' I

SS' I

$9 S^{\prime} \mathrm{I}$

$\angle S^{\prime} I$

$0 \angle ' \mathrm{I}$

$\mathrm{I} L \cdot \mathrm{I}$

$\mathrm{ZL} \cdot \mathrm{I}$

$\varepsilon L ' \mathrm{I}$ -

$\downarrow L ' I$

$6 \varepsilon^{\prime} \varepsilon$

โ胡

乙ち' $\varepsilon$

$\angle \nabla^{\prime} \varepsilon$

$8 t^{\circ} \varepsilon$

$6 b^{\circ} \varepsilon$

IS' $\varepsilon$

乙S. $\varepsilon$

乙S' $\varepsilon$

$\varepsilon S^{\prime} \varepsilon-$

$\varepsilon S^{\prime} \varepsilon$

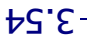

$9 S^{\prime} \varepsilon$

$9 S^{\prime} \varepsilon$

$\angle S^{\prime} \varepsilon$

$8 \varsigma^{\prime} \varepsilon-$

$8 S^{\prime} \varepsilon-$

6S' $\varepsilon$

$6 S^{\prime} \varepsilon-$

$6 \mathcal{S}^{\prime} \varepsilon$

$09^{\circ} \varepsilon$

$09^{\circ} \varepsilon$

ح9' $\varepsilon$

乙L' $\varepsilon$

$\downarrow L ' \varepsilon$

$S L \cdot \varepsilon$
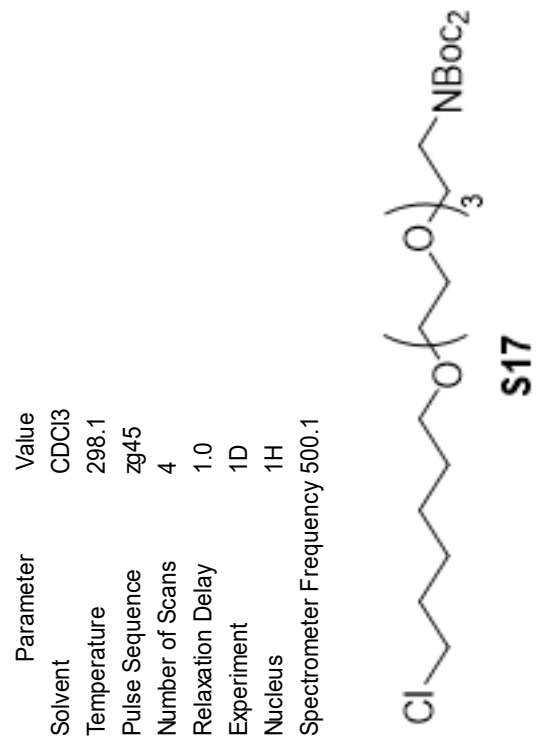

$\infty$

웅

ก

응

웅

웅

윽

음

웅

기 
Bioorthogonal retro-Cope elimination reaction of N,N-dialkylhydroxylamines and strained alkynes Dahye Kang and Justin Kim*
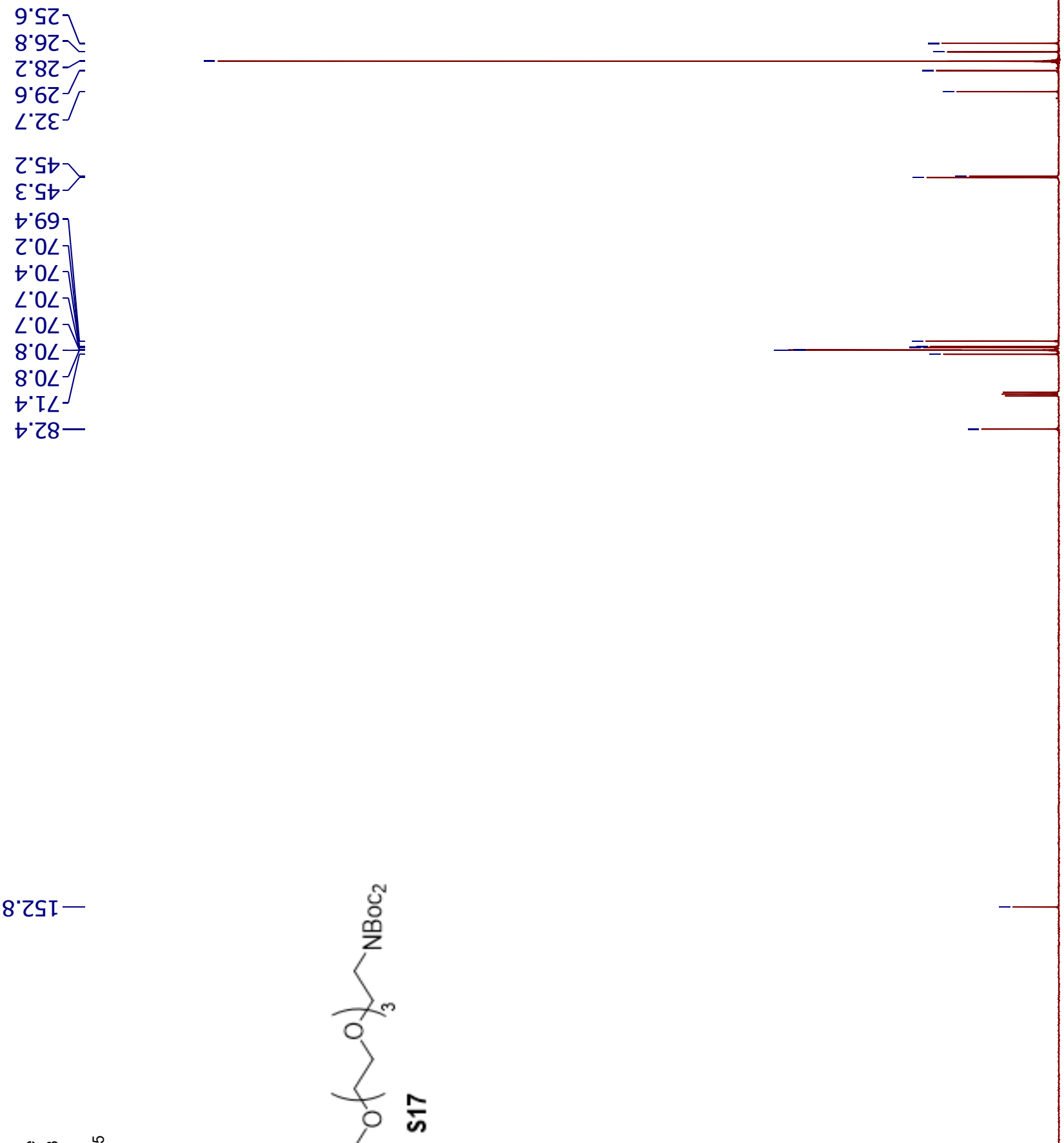

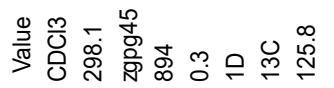
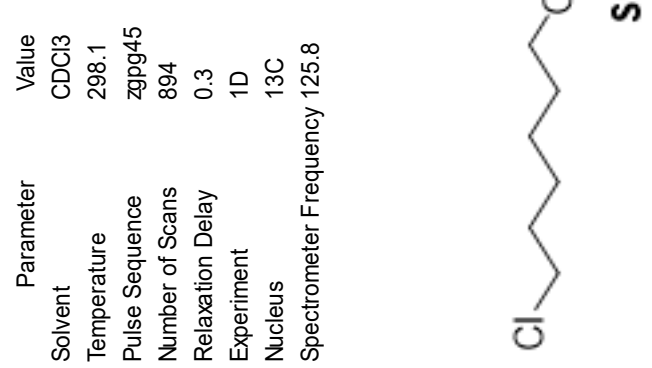

8 

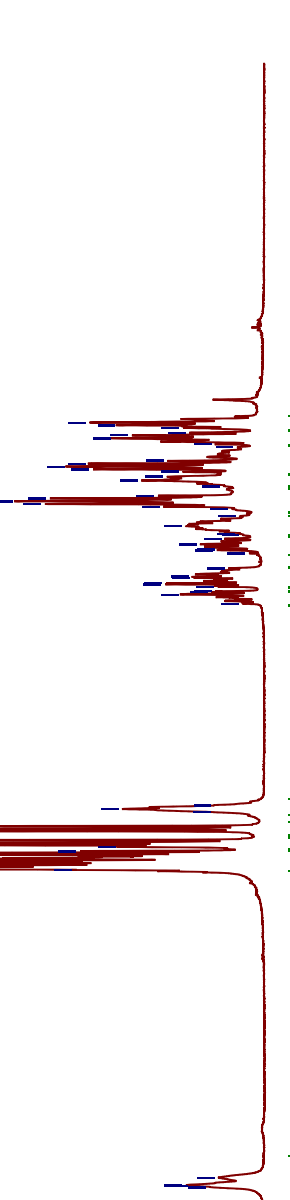

斯
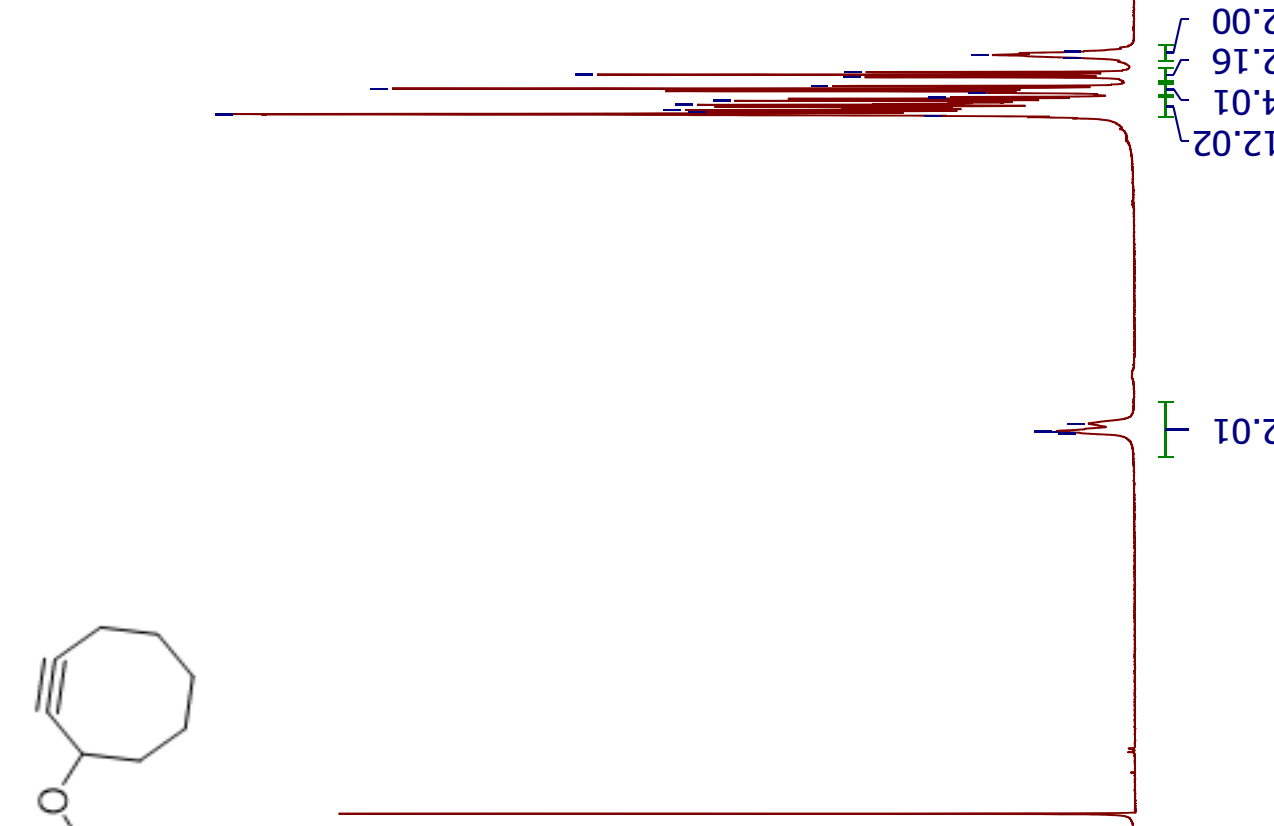

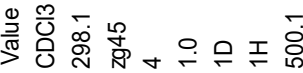<smiles>[C]1=CCC=C=C1</smiles><smiles>[3H][CH]</smiles>

(n)




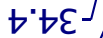

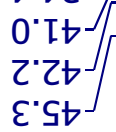

乙: $\angle 9$

$\varepsilon^{\prime} 0<$

$\succ^{\circ} 0 \angle$

$\mathrm{S}^{\circ} 0<$

$8.0<$ 原

$8.0 \angle$

$6^{\circ} 0 \angle$

$6.0<$

$S \cdot T L$

เ. I6-

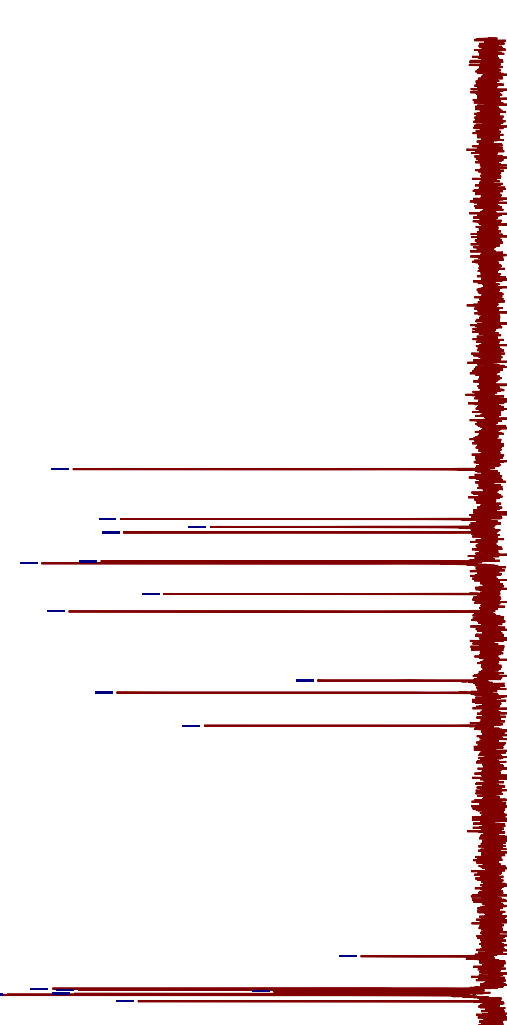

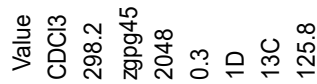

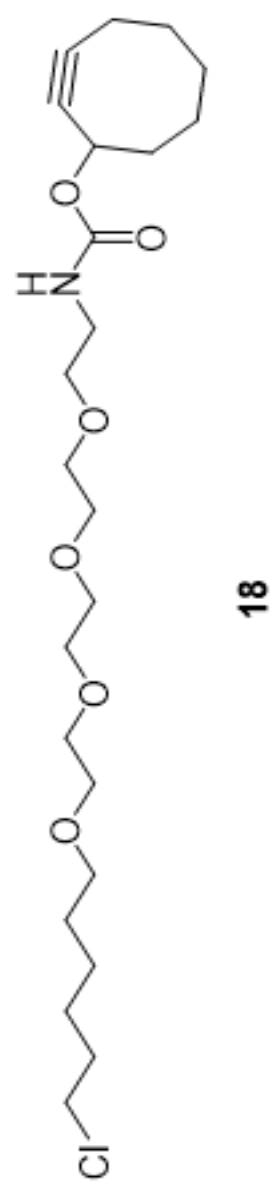


Bioorthogonal retro-Cope elimination reaction of N,N-dialkylhydroxylamines and strained alkynes Dahye Kang and Justin Kim*

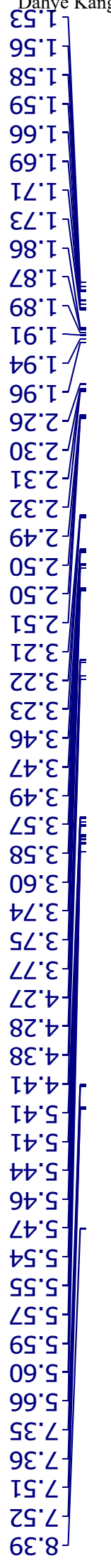
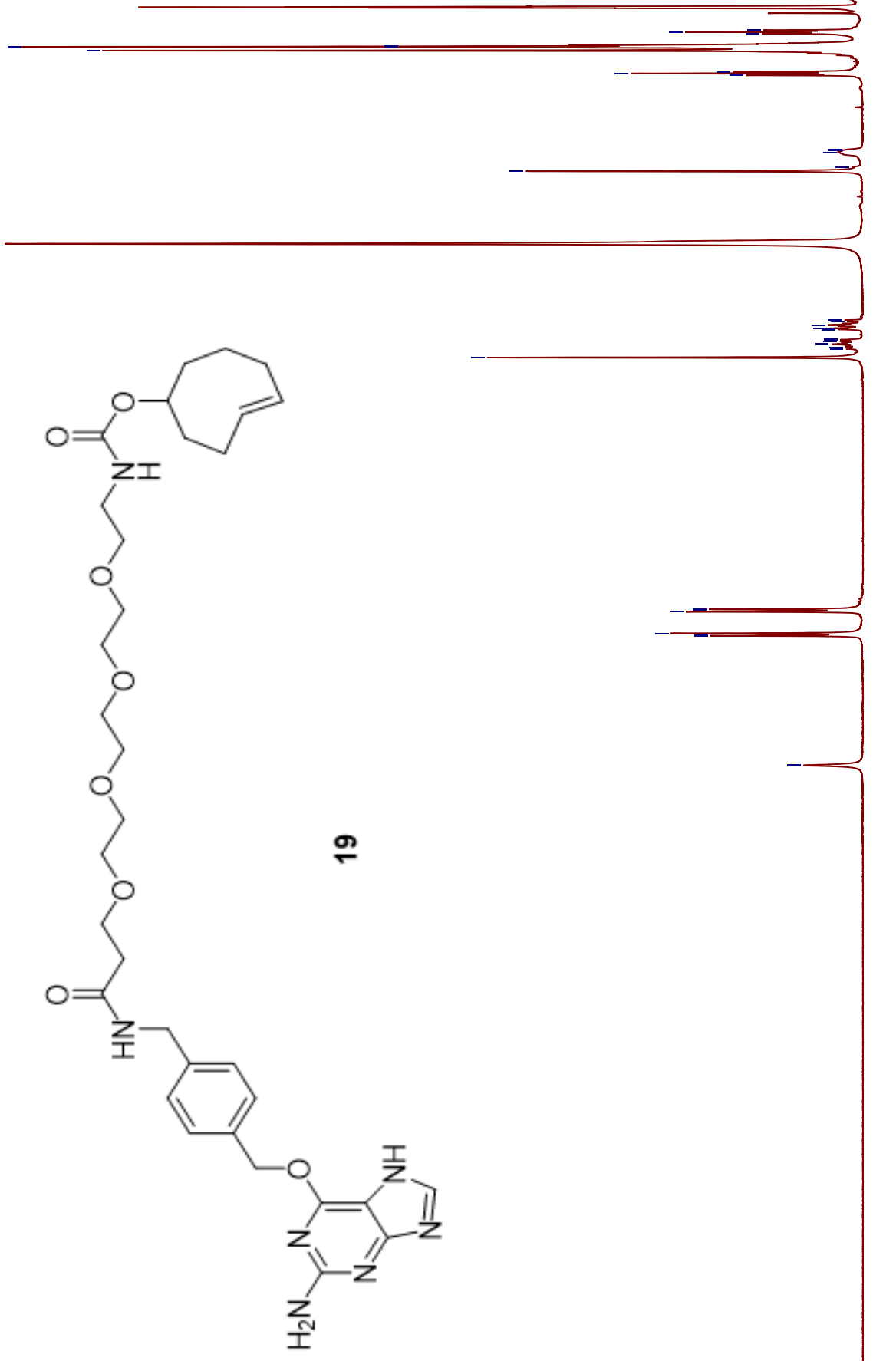

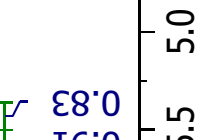

竞

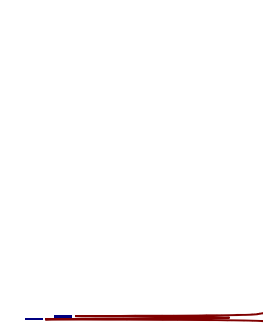

门。

ขอ

옹

T- 90.

+ $\varepsilon 0^{\circ}$

-

는

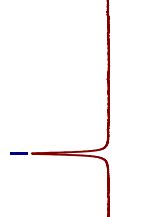

I

$-\infty$

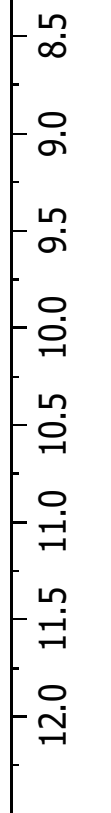



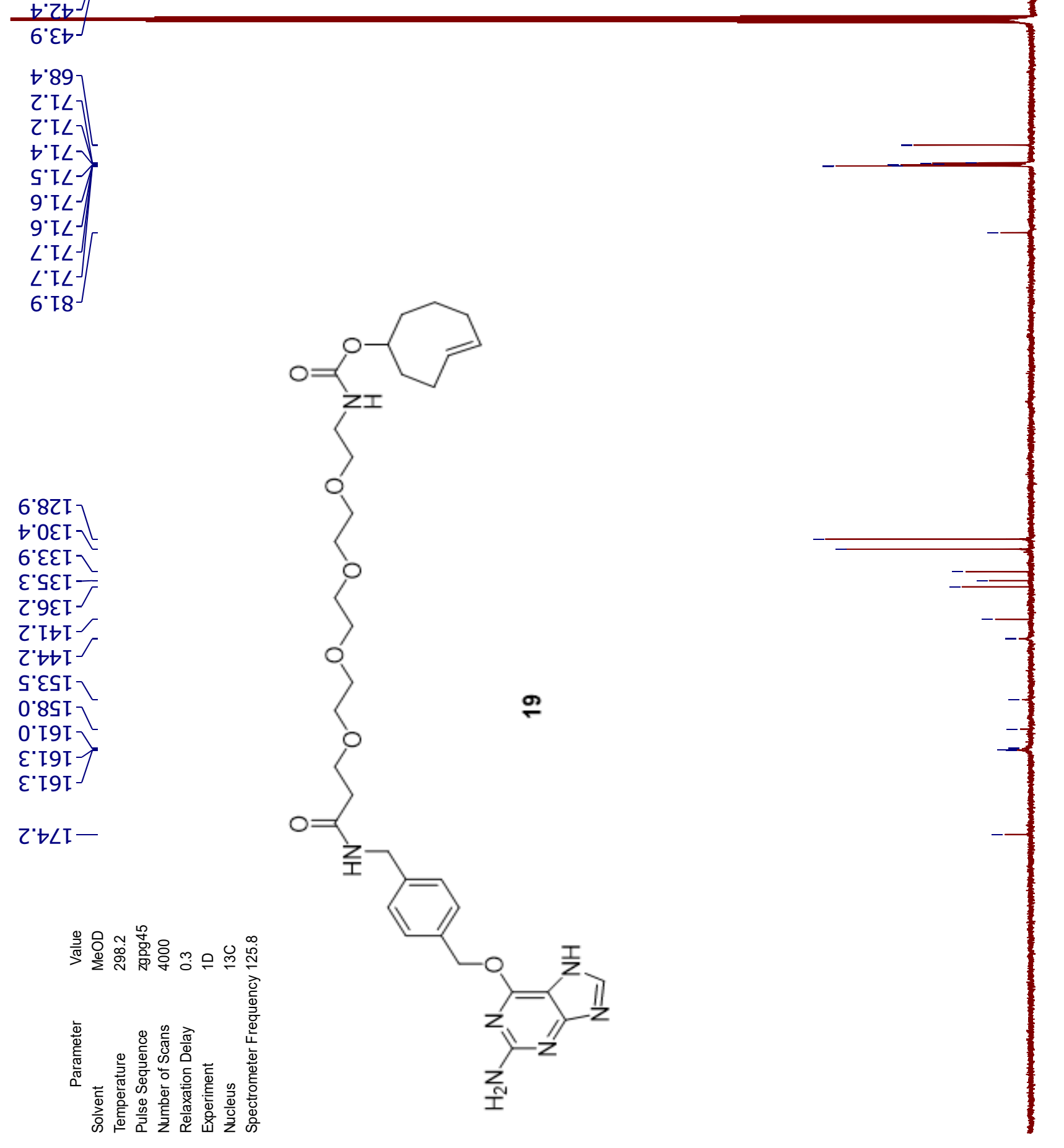

$\varepsilon$ '

$6^{\circ} \angle \varepsilon \backslash$

$8^{\circ} 6 \varepsilon-$

L'功底

6.E๖

๑.89

C' $\mathrm{L} L$

2'I $\angle \sqrt{ }$

$t \cdot T<$

9. $T \angle$

$9^{\prime} \angle \angle$

$\angle \cdot T \angle \cdot$

$\angle \cdot T L$

6. 18 